\title{
Norbert Rouland (1979)
}

\section{Les modes juridiques de solution des conflits chez les Inuit}

\begin{abstract}
Un document produit en version numérique par Mme Marcelle Bergeron, bénévole Professeure à la retraite de l’École Dominique-Racine de Chicoutimi, Québec Courriel: mabergeron@videotron.ca

$$
\text { Page web }
$$
\end{abstract}

Dans le cadre de la collection: "Les classiques des sciences sociales" Site web: http://classiques.uqac.ca/

Une collection développée en collaboration avec la Bibliothèque Paul-Émile-Boulet de l'Université du Québec à Chicoutimi Site web: http://bibliotheque.uqac.ca/ 


\section{Politique d'utilisation de la bibliothèque des Classiques}

Toute reproduction et rediffusion de nos fichiers est interdite, même avec la mention de leur provenance, sans l'autorisation formelle, écrite, du fondateur des Classiques des sciences sociales, JeanMarie Tremblay, sociologue.

Les fichiers des Classiques des sciences sociales ne peuvent sans autorisation formelle:

- être hébergés (en fichier ou page web, en totalité ou en partie) sur un serveur autre que celui des Classiques.

- servir de base de travail à un autre fichier modifié ensuite par tout autre moyen (couleur, police, mise en page, extraits, support, etc...),

Les fichiers (.html, .doc, .pdf, .rtf, .jpg, .gif) disponibles sur le site Les Classiques des sciences sociales sont la propriété des Classiques des sciences sociales, un organisme à but non lucratif composé exclusivement de bénévoles.

Ils sont disponibles pour une utilisation intellectuelle et personnelle et, en aucun cas, commerciale. Toute utilisation à des fins commerciales des fichiers sur ce site est strictement interdite et toute rediffusion est également strictement interdite.

\section{L'accès à notre travail est libre et gratuit à tous les utilisateurs. C'est notre mission.}

Jean-Marie Tremblay, sociologue

Fondateur et Président-directeur général, LES CLASSIQUES DES SCIENCES SOCIALES. 
Un document produit en version numérique par Mme Marcelle Bergeron, bénévole, professeure à la retraite de l’École Dominique-Racine de Chicoutimi, Québec.

Courriel : marcelle_bergeron@uqac.ca; mabergeron@videotron.ca

\section{Norbert Rouland}

Les modes juridiques de solution des conflits chez les Inuit.

Études Inuit/Studies, vol. 3, numéro hors-série, 1979, 171 pp. Québec : Département d'anthropologie, Université Laval.

[Autorisation formelle accordée par l'auteur le 11 janvier 2011 de diffuser cette œuvre dans Les Classiques des sciences sociales et autorisation confirmée par l’éditeur le 14 janvier 2011.]

gf Courriel : norbert.rouland@wanadoo.fr

Polices de caractères utilisés :

Pour le texte : Times New Roman, 12 points.

Pour les citations : Times New Roman 10 points.

Pour les notes de bas de page : Times New Roman, 10 points.

Édition électronique réalisée avec le traitement de textes Microsoft Word 2008 pour Macintosh.

Mise en page sur papier format : LETTRE US, 8.5'” x 11’’.

Édition complétée le 3 août 2011 à Chicoutimi, Ville de Saguenay, Québec. 


\section{Norbert Rouland}

(1979)

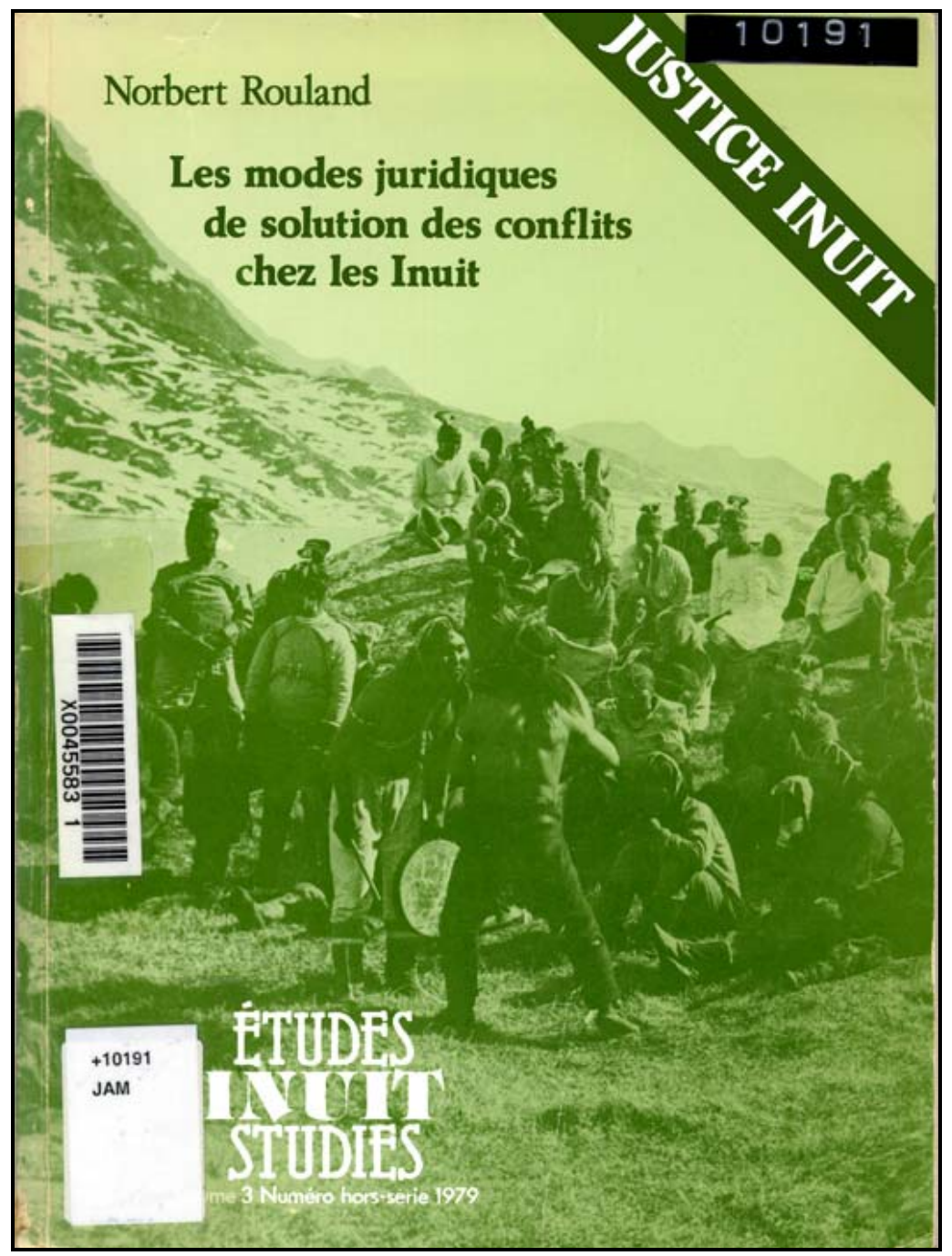

Études Inuit/Studies, vol. 3, numéro hors-série, 1979, 171 pp. Québec : Département d'anthropologie, Université Laval. 


\section{Quatrième de couverture}

\section{Les modes juridiques de solution des conflits chez les Inuit}

Les sociétés arctiques sont aujourd'hui un des centres de l'actualité. La crise de l'énergie a suscité envers les territoires du Nord et ceux qui les peuplent un intérêt où l'érudition, la curiosité, et la stratégie du développement ont chacune leur part. Norbert Rouland, qui enseigne l'histoire du droit à l'Université d'Aix-en-Provence, est déjà l'auteur d'un livre sur la politique suivie par le gouvernement du Québec à l'égard des Inuit (Les Inuit du Nouveau-Québec et la convention de la baie James, Québec, Association Inuksiutiit et Centre d'Études Nordiques, 1978). Il se penche dans ce nouvel ouvrage sur les structures juridiques et sociales traditionnelles. L'auteur envisage les différents modes suivant lesquels le droit coutumier inuit assure le règlement des conflits internes. La première partie de l'ouvrage différencie les critères du droit inuit de ceux du droit occidental en tentant d'y constituer une anthropologie. Puis l'auteur décrit et interprète les différents processus concrets (contrôle de la vendetta, duel de chants, etc.) employés par les sociétés inuit depuis le Groenland jusqu'à l'Alaska pour maîtriser les conflits qui se déclenchent en leur sein. Une dernière partie présente et commente quelques textes ethnographiques (traduits de K. Rasmussen) illustrant les réflexions théoriques de l'auteur à partir de cas concrets tirés de l'expérience de ces sociétés.

\section{Solving disputes among the Inuit}

Arctic societies are increasingly coming to the forefront of public affairs. Interest for northern territories and their inhabitants - in which scholarship, curiosity and development strategy are mixed, - has been aroused by the energy crisis. Norbert Rouland, who teaches history of law at the University of Aix-enProvence has already published a book about the policy adopted by the government of Quebec vis-à-vis the Inuit (Les Inuit du Nouveau-Québec et la convention de la Baie James, Québec, Association Inuksiutiit et Centre d'Études Nordiques 1978). In this new book he analyzes traditional social and juridical structures and examines the various ways in which internal conflicts are settled within Inuit customary law. In the first part of the book the criteria of Inuit law are distinguished from those of Western law from an anthropological standpoint. The author then goes on to describe and explain the actual processes that have been used by Inuit societies from Greenland to Alaska, in order to regulate conflicts arising in their midst (social control of vendettas, song duels, etc.). In the last section ethnographic documents are presented and discussed to illustrate the theoretical views of the author on the basis of actual cases drawn from the life of these societies. 


\section{NOTE DE L'ÉDITEUR}

Ce texte a été écrit en 1976 et publié sous forme polycopiée à Aix-enProvence (France), en deux livraisons successives. L'une (mai 1976) comportait la première partie du présent ouvrage (La sanction du droit chez certaines populations esquimaudes : problèmes théoriques et modes d'intervention de la communauté dans les procédures de règlement des conflits); l'autre (novembre 1976) correspond à la seconde partie (Textes esquimaux traduits et commentés : chants et duels judiciaires). Docteur en droit et ès science politique, l'auteur est maîtreassistant à la Faculté de Droit d'Aix-en-Provence. 
Lac superbe, charriant des glaçons, Rempli de glace malgré le soleil, Une langueur pénible frappe mes membres

Et je me repose,

Tandis que je goûte les joies indicibles Éparses sur tes rives.

(Chant d'un jeune garçon esquimau recueilli par K. Rasmussen, (Du Groënland au Pacifique)

On ne peut pas connaître un pays par la simple science géographique ... On ne peut, je crois, rien connaître par la simple science ; c'est un instrument trop exact et trop dur. Le monde a mille tendresses dans lesquelles il faut se plier pour les comprendre avant de savoir ce que représente leur somme ... Seul le marin connaît l'archipel.

(Jean GIONO, L'eau vive) 
[p. 3]

\section{TABLE DES MATIÈRES}

PREMIÈRE PARTIE :

LA SANCTION DU DROIT CHEZ CERTAINES POPULATIONS INUIT : PROBLÈMES THEORIQUES ET MODES D'INTERVENTION DE LA COMMUNAUTÉ DANS LES PROCÉDURES DE RÈGLEMENT DES CONFLITS

INTRODUCTION de la première partie

CHAPITRE I. LES CARACTÈRES SPÉCIFIQUES DE LA SANCTION DU DROIT CHEZ LES POPULATIONS ESQUIMAUDES

Section I : $\quad$ LES TRAITS GENÉRAUX DE LA SANCTION : CORRECTIONS ET ÉLARGISSEMENT DE LA THÉORIE CLASSIQUE

Section II : LES MODES SOCIO-PSYCHOLOGIQUES DE SANCTION : PRÉÉMINENCE DU GROUPE

A) Le rôle des maïeutes et la structure coutumière du droit esquimau

a) Prééminence de la coutume

b) Les principaux maïeutes

1) Le shaman

2) Leaders civils

B) Types de procédures de sanction du droit socio-psychologiques

CHAPITRE II. ACTION DU GROUPE ET INTERÊT PUBLIC DANS LES PROCESSUS JURIDIQUES INSTITUTIONNELS DE CONTRAINTE

Section I. MODE PRIVÉ DE SOLUTION DES CONFLITS : LA VENGEANCE FAMILIALE ET SA LIMITATION PAR LE GROUPE

A) Les causes

B) Les modalités

1) L'ordre des vengeurs

2) Les modalités d'exécution

3) Effets annexes de la vengeance

C) Les limitations apportées par le groupe à la vendetta 
Section II. MODALITÉS DE L'ACTION PUBLIQUE : INTERVENTION DIRECTE DU GROUPE DANS LE RÈGLEMENT DES CONFLITS

A) La décision communautaire de liquidation physique de l'ennemi public

B) Les duels judiciaires

1) Principes généraux

2) Juridicité des duels judiciaires chez les Inuit : l'exemple fourni par les compétitions de chants

a) Le mécanisme de la compétition de chants et le régime de la preuve

b) Caractère "public" des compétitions de chants : le rôle de la communauté

c) La finalité des compétitions de chants

C) Éléments d'une anthropo-géographie des compétitions de chants

CONCLUSION de la première partie: DE L'HISTOIRE DU DROIT À L'ETHNOLOGIE : MOI ET L'AUTRE

\section{DEUXIÈME PARTIE :}

TEXTES INUIT TRADUITS ET COMMENTÉS. CHANTS ET DUELS JUDICIAIRES

INTRODUCTION de la deuxième partie

TEXTE I : $\quad$ Chants et duels judiciaires

TEXTE II : $\quad$ Une étrange danse au tambour

TEXTES III : Chants à objet détourné

TEXTES IV: Chants à objet direct

TEXTES V : Chants à objet mixte

TEXTES VI : Chants à caractère particulier

TEXTES VII : Chants non-judiciaires

\section{BIBLIOGRAPHIE}


[p. 6]

\section{PREMIÈRE PARTIE}

La sanction du droit

chez certaines

populations inuit

\section{Problèmes théoriques \\ et modes d'intervention \\ de la communauté \\ dans les procédures \\ de règlement des conflits}

$\underline{\text { Retour à la table des matières }}$ 
[p. 7]

\section{INTRODUCTION DE LA PREMIÈRE PARTIE}

$\underline{\text { Retour à la table des matières }}$

L'univers juridique des populations archaïques est difficilement accessible à la pensée occidentale dont les déterminants économiques et socio-politiques se sont constitués dans des contextes souvent fort différents. L'idée même que ces groupes humains puissent posséder un système de normes sociales élevé à la dignité d'obligations juridiques n'est pas acceptée par tous les auteurs, au moins pour les plus archaïques de ces populations ${ }^{1}$. Ces visions trop étroites pèchent souvent par ethnocentrisme : le phénomène juridique n'est pas réductible dans son entier aux formes sous lesquelles il s'exprime dans les sociétés occidentales industrialisées $^{2}$, qui [p. 8] ont subi la double influence de la tradition romaniste et de la

1 R. MASPETIOL (La société politique et le Droit (Paris, 1957), 3) note que chez les Inuit, à l'instar des Pygmées, Lembas et Lakas, "De telles structures (politiques élémentaires) ne paraissent pas supérieures à celles des sociétés animales constituant les groupements homogènes les plus évolués". Pour P. AMSELEK (Perspectives critiques d'une réflexion épistémologique sur la théorie du droit (essai de phénoménologie juridique) (Thèse Droit Paris 1962, Paris, 1964), 174 et $n .172$ ), il semble que le phénomène juridique soit absent chez les sociétés inuit qui, comme les sociétés animales, connaîtraient plutôt des mécanismes régulateurs spontanés, biologiques. On reste évidemment frappé de stupeur devant la dose d'ethnocentrisme - ou d'ignorance - que supposent de tels jugements. Ils ne sont pourtant pas exceptionnels : pour G. BURDEAU, étant donné que la mentalité primitive n'abstrait pas, l'individu ne peut réfléchir sur les impératifs posés par sa coexistence avec les autres membres du groupe : "... ce serait une aberration que de prétendre trouver en lui les éléments, même rudimentaires, d'un acte d'intelligence à propos des destinées collectives ... il ne peut être sujet du droit, car l'opinio necessitatis, qui est une des conditions de l'autorité du droit, ne peut exister que chez un homme capable de comprendre qu'il a à obéir à une règle ou à subir une contrainte ..." (G. BURDEAU, Traité de Science Politique, I : Le Pouvoir Politique (Paris, 1966), 51 et n. 2). Toujours dans le sens de l'inexistence du droit chez les Inuit, cf. T.B. BOTTOMORE, Introduction à la sociologie, (Paris, 1974), 258.

2 "... le juriste qui ne serait que juriste se condamne à déformer la réalité qu'il enferme dans des formules ou des concepts non pertinents (...) le juriste ... avant d'aborder des sociétés autres, devra dépasser sa propre formation, la mettre sociologiquement en situation, et comprendre ce que représente une objectivité totale (...) cette objectivité, on le sait, vise au dépassement de certaines catégories logiques qu'on croit trop facilement universelles" (J. POIRIER, "Introduction à l'ethnologie de l'appareil juridique", dans Ethnologie Générale, sous la direction de J. POIRIER (Paris, 1968), 1099). À titre d'exemple concret, on sait combien sont grandes les difficultés à établir la nature et les composants du droit de propriété des Inuit sur leurs terres, définition... rendue pressante par l'industrialisation des régions arctiques. Nous avons essayé ailleurs de montrer que ces difficultés étaient, en grande partie, dues à des différences radicales dans l'analyse faite du droit de propriété par le droit occidental et les droits indigènes (Cf. N. ROULAND, "Le droit de propriété des Esquimaux et son intégration aux structures juridiques 
culture chrétienne. Dans ces sociétés, un appareil institutionnel développé incite la réflexion doctrinale à définir le droit par des critères formels, le plus souvent liés à l'existence d'organes étatiques. Parmi ces critères, celui de la sanction occupe une place cardinale ${ }^{1}$ : c'est à la façon dont une règle de conduite sociale est rendue obligatoire pour les membres du groupe qu'on lui reconnaît son caractère juridique. Si ce principe, quoiqu'exprimé de façon souvent trop absolue et dogmatique, n'est pas inexact dans son fondement, on doit le préciser singulièrement pour pouvoir l'adapter aux phénomènes juridiques des sociétés archaïques. En effet, dans ces sociétés souvent a-étatiques, les mécanismes de sanction revêtent une forme plus diffuse - quoique non moins efficace - que dans les nôtres : c'est tout particulièrement le cas des sociétés Inuit, que nous allons étudier dans les lignes qui suivent.

[p. 9] Outre le caractère spécifique qu'elles ont su donner aux processus de sanction de leurs règles de droit, ces sociétés ont eu à résoudre le difficile problème du leadership, dans le cadre de groupes qui ne connaissent pas la notion d'état, et récusent celle de chef. Il ne nous appartient pas ici d'étudier cette difficile question, mais seulement de nous pencher sur les conséquences qu'entraîne cette conception originale du pouvoir sur l'application des règles de droit. Déduire de l'absence de chefs formels et dotés de pouvoirs codifiés et permanents l'inexistence de l'autorité publique serait, en effet, un grave contre-sens : nous verrons que le groupe, directement ou par l'intermédiaire de certaines autorités, intervient très activement dans les processus de contrainte juridique, soit en posant des limitations aux modes privés de solution des conflits au nom de l'intérêt public, soit en mettant en œuvre de véritables types d'action publique.

occidentales : problèmes d'acculturation juridique", Actes $d u$ XLII ${ }^{e}$ Congrès International des Américanistes V, (Paris, 1978), 123-140.

Cf. par exemple, les définitions de la règle de droit données par les traités de droit civil récents :

- "La règle de droit est sanctionnée par la contrainte. Il y a là un caractère spécifique de la règle de droit. Une règle qui ne serait pas obligatoire ne serait pas une règle de droit (...) la règle de droit est une règle de conduite sociale qui, sanctionnée par la contrainte, doit avoir pour but de faire régner l'ordre en donnant la sécurité dans la justice" (H.L., J. MAZEAUD, Leçons de droit civil, I (Paris, 1965), 23,26). Ces auteurs adjoignent bien à la notion de contrainte un critère de fond tiré de la finalité de la règle de droit, mais la référence est plus morale que juridique : ils définissent ce que la règle de droit devrait être, et non pas forcément ce qu'elle est.

- "(La règle de droit) est une règle de conduite humaine, à l'observation de laquelle la société peut nous contraindre par une pression extérieure plus ou moins intense" (J. CARBONNIER, Droit Civil, I (Paris, 1965), 51. Sur les dangers de l'ethnocentrisme, cf. également E. LE ROY, "Justice africaine et oralité juridique", dans Bull. de l'I.F.A.N., XXXVI - B, 3 (1974), 563. 
Nous ordonnerons donc nos propos en deux parties :

CHAPITRE I Les caractères spécifiques de la sanction du droit chez les populations Inuit.

CHAPITRE II Action du groupe et intérêt public dans les processus juridiques institutionnels de contrainte. 
[p. 10]

\section{CHAPITRE PREMIER}

\section{LES CARACTÈRES SPÉCIFIQUES DE LA SANC- TION DU DROIT CHEZ LES POPULATIONS INUIT}

\section{$\underline{\text { Retour à la table des matières }}$}

La règle juridique n'est que le produit de la société qu'elle prétend organiser ${ }^{1}$ : il est donc normal que les modalités de sa sanction s'accordent aux principes fondamentaux de conduite sociale en vigueur dans la société considérée. La conséquence en est qu'une fois reconnue l'importance de la sanction comme élément de repérage du juridique, les processus de contrainte ne sont pas tous réductibles à un modèle universel, mais au contraire inhérents à une société donnée.

Dans le cas des sociétés Inuit - et cela sans négliger les variations régionales sur lesquelles nous reviendrons en détail plus loin ${ }^{2}$ - les mécanismes de contrainte psychologique sont à ce point importants qu'ils méritent qu'on les étudie isolément.

Nous aborderons donc, dans ce chapitre, successivement deux problèmes : les traits généraux de la sanction, les modes socio-psychologiques de sanction.

$1 \quad$ "... le droit ne peut être envisagé dans une Histoire des institutions, comme une réalité en soi, évoluant pour des raisons propres, et étudié d'un point de vue intrinsèque (...) le droit apparaît comme l'expression stabilisée, rationalisée à un moment donné, de rapports sociaux et économiques de doctrines et de tendances idéologiques, d'interventions de groupes dominants ou de personnalités décisives" (J. ELLUL, Histoire des Institutions : l'Antiquité, (Paris, 1972), avantpropos).

2 Cf. infra, pp. 42-43, 96-101. 


\section{Section I : LES TRAITS GÉNÉRAUX DE LA SANCTION : CORRECTIONS ET ÉLARGISSEMENT DE LA THÉORIE CLASSIQUE}

$\underline{\text { Retour à la table des matières }}$

La majorité des auteurs s'accorde à donner à la sanction le rôle principal comme élément de reconnaissance du juridique ${ }^{1}$. Pour n'être pas erroné, ce point de vue doit être éclairé par certaines remarques complémentaires.

[p. 11] La sanction ne peut, à elle seule, définir le droit, qui nécessite l'emploi conjoint d'une batterie de critères : nous avons ailleurs ${ }^{2}$ étudié ce problème et tenté de dresser une typologie critique des différents critères du droit.

La référence exclusive à ce critère traduit, d'ailleurs, un ethnocentrisme plus ou moins conscient. Jean Carbonnier a fort justement dénoncé cet excès :

"Des siècles de gouvernement policé, rationnel, efficace, ont formé les esprits à faire de la règle l'essentiel du droit, et de la sanction par laquelle son exécution est assurée, une de ses dimensions nécessaires (...). Ceux qui ne conçoivent le droit qu'à travers sa sanction, ne mesurent pas toujours l'ambigüité inhérente à leur critère. Affirmer, en effet, qu'une règle est juridique si, transgressée, elle déclenche un certain mécanisme de contrainte qui tendra à la ramener à exécution c'est reconnaître implicitement qu'elle peut être transgressée... La possibilité de la transgression devient ainsi beaucoup plus importante que la possibilité de la contrainte, et le défi lancé par le transgresseur à la règle apparaît comme le moment décisif dans le mécanisme du droit (...) Ce serait la possibilité d'une mise en question qui caractériserait le mieux la norme juridique, une certaine nature interrogative" ${ }^{3}$.

Voir en la sanction le point essentiel de cristallisation du phénomène juridique ainsi que l'élément de sa définition peut, en effet, trop souvent conduire à une mauvaise méthode d'approche du phénomène juridique dans les sociétés archaï-

1 "Le fait juridique se définit par la nature de la sanction applicable à la transgression (...). L'existence du droit ... est donc conditionnée par l'existence de la sanction juridique". (J. POIRIER, op. cit., 1092-1093) ; cf. supra, p. 8 et n. 1.

2 Cf. N. ROULAND, Approche du phénomène juridique dans les sociétés traditionnelles et introduction à l'étude du droit esquimau à la lumière des données comparatives fournies par les droits archaïques de l'Antiquité occidentale, (Texte de conférences prononcées au Centre d'Études Arctiques, École des Hautes Études en Sciences Sociales, sous la direction du Pr Jean MALAURIE ; Paris, 1975), 2241 et, surtout, 3235. Cf. également les analyses d'E. LE ROY, Méthodologie de l'anthropologie juridique (Paris I, Séminaire d'anthropologie juridique, Document pédagogique $\mathrm{n}^{\circ} 1$ ), 35-38.

3 J. CARBONNIER, Sociologie Juridique, (Paris, 1972), 134-135. 
ques ${ }^{1}$. À notre sens, on peut critiquer ce point de vue - dans sa manifestation la plus exclusive - à un double niveau :

[p. 12]

- la sanction n'est, au mieux, qu'un élément de repérage du juridique, et non pas un critère du droit ${ }^{2}$ : pas plus que le symptôme n'est la maladie, le droit n'est réductible à ses mécanismes de contrainte ${ }^{3}$. L'effet n'est pas la cause. Cependant, la sanction des obligations juridiques possède certains caractères ${ }^{4}$ qui la spécifient par rapport aux autres règles de conduite sociale : en cela, elle peut être comme un repère.

- Il y a plus grave, et nous en venons maintenant à l'accusation d'ethnocentrisme que nous formulions plus haut ${ }^{5}$ : privilégier la sanction dans la définition du droit, c'est en fait privilégier les types de sociétés - qui sont les nôtres - qui ont fait essentiellement du droit un mécanisme de contrainte. En ce sens, du point de vue de la sociologie juridique, il est parfaitement normal que la pensée juridique occidentale ait donné à ce critère de la sanction cette importance exagérée, car elle se référait inconsciemment exclusivement aux systèmes sociopolitiques qui l'avaient engendrée. Mais lorsqu'on se tourne vers les sociétés archaïques, on s'aperçoit vite que, dans les procédures de sanction, l'essentiel n'est pas l'exécution de la règle, mais le rétablissement de l'ordre public, de la paix sociale. La peine est dirigée moins contre le criminel que contre le crime lui-même ${ }^{6}$.

[p. 13]

1 À titre comparatif, on lira avec intérêt : E. LE ROY, op. cit., 564-569; Cours d'Histoire des Institutions d'Afrique Noire : I : Le Pouvoir politique en Afrique Noire (Brazzaville, 1972-73), 152-167.

2 Cf. N. ROULAND, op. cit., (Approche du phénomène juridique...), 32-35.

3 "Sanction, contrainte, apparaissent donc, en dernière analyse, comme des éléments de la règlementation juridique à définir ; elles ne sauraient logiquement constituer une définition de son objet par la science du droit. Tout au plus, elles imposent à celle-ci une constatation d'ordre historique : à savoir que l'on retrouve, comme une constante dans les systèmes juridiques, l'institution de mécanismes de juridiction et de coercition, des règles prévoyant des mécanismes sanctionnateurs ou des recours à la contrainte physique". (Lucien AULAGNON, Aperçu sur la force dans la règle de droit, dans Mél. Roubier (Paris, 1961), 29 ; cité par P. AMSELEK, Perspectives critiques d'une réflexion épistémologique sur la théorie du droit, Essai de Phénoménologie juridique (Thèse Droit Paris 1962 ; Paris, 1964) ; "Le caractère contraignant n'est certes pas un simple hasard dans l'évolution du droit ; il n'est pas néanmoins essentiel, au point d'être le signe de la juridicité" (J. CARBONNIER, op. cit., 134).

Sur ces caractères, cf. infra, pp. 16-20.

Cf. supra, p. 7.

" ... l'étude ethnologique révèle, en mainte société archaïque, une pensée qui, faisant contraste avec la nôtre, traite le droit non pas comme une règle dont l'exécution devrait être imposée par la force, mais ... comme une paix, une concorde, un équilibre à gagner par la conciliation et la réconciliation", (J. CARBONNIER, op. cit., 134). Dans le même sens, cf. H. LEVY-BRUHL, "L'Ethnologie juridique", dans Ethnologie générale, op. cit., 1162, 1170-1171 ; A. CUVILLIER, Manuel de sociologie, II, (Paris, 1968), 493. 
On trouve des traces de cette conception dans les institutions judiciaires de la Rome archaïque ; le terme même de ius exprime la volonté des dieux, ce qui est licite par rapport aux dieux ; le droit est le résultat de l'accord entre la volonté des hommes et l'ordre du monde tel qu'il est voulu par les dieux ${ }^{1}$. Le roi est d'ailleurs censé exercer son activité judiciaire sous l'inspiration divine ${ }^{2}$. Cette conception de la fonction du droit comme le moyen de rétablir un ordre supérieur perturbé par la transgression des règles se retrouve encore dans la procédure archaïque du Sacramentum, bien connue des romanistes ${ }^{3}$. C'est la plus ancienne procédure de jugement, qui pouvait être utilisée pour tout litige en matière civile. Elle est fort intéressante par ce qu'elle nous apprend sur la mentalité archaïque romaine. En effet, la solution du litige n'est pas donnée directement par l'examen des faits qui en sont l'objet, mais par un "détour" faisant intervenir les dieux. Chacune des parties au procès affirme contradictoirement son droit sur la chose contestée, en assortissant sa déclaration d'un serment à caractère religieux : celui qui aurait agi sans droit encourait la sacralité, c'est-à-dire qu'il était voué aux dieux infernaux. La solution du litige était donc d'abord religieuse, et liée à la valeur de ce serment, qui ne pouvait être reconnue que par une réponse divine, donnée par les augures, qui étaient les interprètes de la volonté des dieux. Le coupable est certes châtié, puisque devenu sacer, il peut être tué sans que son meurtrier soit inquiété. Cependant, la perception de la transgression de la règle comme celle d'un ordre supérieur dont l'harmonie doit être rétablie est ici évidente.

Selon des modalités évidemment différentes, les populations inuit, dans leur mode de résolution des conflits, témoignent d'un souci identique de préservation des équilibres fondamentaux. Ainsi, la véritable faute consiste [p. 14] beaucoup plus dans la récidive que dans l'accomplissement d'un acte isolé ${ }^{4}$, car la répétition des infractions peut mettre en jeu la vie de la communauté. Dans les sociétés inuit pauvres, où la marge de survie du groupe est si étroite que les perturbations doivent être réduites au minimum, les conflits reçoivent des procédures de sanction réglées de façon à économiser les vies humaines ${ }^{5}$. Nous verrons plus loin ${ }^{6}$ que, dans ces duels judiciaires et compétitions de chants, l'essentiel du débat ne porte pas sur les faits litigieux : ces compétitions ont pour objet de permettre un défoulement aux parties en conflit ${ }^{7}$, et le "vainqueur" n'est pas forcément celui qui est dans son bon droit. L'objectif principal est, en effet, de rétablir les équilibres internes au sein du groupe ; pour y parvenir, la démarche est différente de celle à laquelle nos systèmes judiciaires nous ont accoutumés en se fixant pour but de

Cf. J. ELLUL, op. cit., 235.

Cf. J. GAUDEMET, Institutions de l'Antiquité, (Paris, 1967), 271.

Ibid., 407-413 ; cf. J. ELLUL, op. cit., 260-261.

Cf. E. ADAMSON-HOEBEL, The Law of Primitive Man, (Cambridge, 1954), 261 ; N. ROULAND, op. cit., 69-70.

J. MALAURIE, "Les civilisations esquimaudes - Essai d'anthropogéographie", dans Ethnologie régionale II, (La Pléiade, Paris, 1978).

Cf. infra, p. 82 sq.

Cf. N. ROULAND, "Le règlement des conflits dans l'Arctique traditionnel", dans Les Nouvelles Affiches, 1896 (Déc. 1974), 2. 
statuer sur la répartition des torts. Enfin, une place beaucoup plus grande est faite à l'amendement du coupable : divers moyens de pression psychologique sont utilisés avant qu'une action concrète soit décidée contre lui ${ }^{1}$, compte tenu, évidemment, de la gravité de sa faute. Avant de sanctionner l'individu, on tente de l'amener à se sanctionner lui-même ${ }^{2}$.

On voit donc que, là aussi, le droit et sa sanction sont avant tout conçus dans leur référence à un ordre supérieur qu'il s'agit de préserver ou de rétablir. Les sociétés inuit, soumises aux pressions d'un environnement rigoureux, et où l'individu doit constamment se soumettre à la loi du groupe, voient dans l'intérêt collectif du groupe cet ordre supérieur : les perturbations causées au corps social par les agissements de ses membres ne doivent pas dépasser une certaine amplitude, sous peine de mettre en danger la vie même du groupe. Ce principe général reçoit des applications différentes suivant les conditions économiques et démographiques propres à la population inuit considérée. Ainsi, dans les riches sociétés baleinières du détroit de [p. 15] Behring, les conflits sont beaucoup plus meurtriers que dans des régions moins favorisées : sur la côte Est du Groënland, beaucoup plus pauvre, on met, au contraire, l'accent sur les procédures de règlement pacifique des conflits (compétitions de chants, notamment) ${ }^{3}$.

Ces quelques considérations montrent suffisamment que le problème de la sanction du droit chez les populations Inuit doit être abordé de façon très nuancée. En effet, si nous nous résumons, on peut dire que :

- la sanction du droit est une des voies par lesquelles on peut parvenir à la connaissance du phénomène juridique, mais elle n'a pas l'importance exclusive que lui a attachée la pensée juridique occidentale ; d'autre part, le droit ne saurait être défini par sa sanction, mais tout au plus repéré.

- La notion même de sanction du droit peut renvoyer à des systèmes de valeurs différents : dans les sociétés "modernes", la sanction consiste surtout dans l'application forcée de la norme juridique et le châtiment de l'individu dont on aura prouvé la culpabilité ; dans les sociétés archaïques, la sanction consiste avant tout dans le rétablissement d'un ordre supérieur, religieux ou social ${ }^{4}$.

On comprend, dès lors, que la sanction du droit chez les populations inuit possède des caractéristiques spécifiques, rendant inadéquates certaines définitions posées par les anthropologues, non encore détachés d'une certaine vision ethnocentriste. Dans un ouvrage, par ailleurs remarquable, et qui est devenu un des

\footnotetext{
Cf. infra, pp. 40-45.

Cf. N. ROULAND, op. cit., (Approche du phénomène juridique), 33-34.

Cf. infra, pp. 88-89.

Cf. N. ROULAND, op. cit., (Approche du phénomène juridique...), 66-69.
} 
grands classiques de l'anthropologie juridique ${ }^{1}$, E. Adamson-Hoebel définit ainsi le droit :

"Une norme sociale est juridique si, à sa mise à l'écart ou à sa transgression répond, de façon régulière, à titre de menace ou de facto, la mise en œuvre de la force physique par un individu ou un groupe jouissant du privilège, socialement reconnu, de pouvoir adopter un tel comportement" 2 .

[p. 16]

Une telle définition ne peut, à notre sens, être retenue : ou, à tout le moins, on ne peut que constater son imperfection si on tente de l'appliquer aux populations Inuit. En effet :

a) la règle de droit doit s'appliquer de façon régulière : tout en donnant acte à Adamson-Hoebel qu'il s'agit bien, dans sa définition, d'une régularité de principe qui peut souffrir les exceptions inhérentes à tout comportement, il semble malhabile de faire de cette régularité un élément de définition. En effet, on réduit ainsi la règle de droit au contentieux : or le champ du droit est infiniment plus vaste que celui de sa contestation ${ }^{3}$ :

"... de ce que la possibilité toujours latente d'un procès soit inhérente à la notion du juridique, il ne s'ensuit pas que la réalité du droit se confonde avec le contentieux. Une des bases de la sociologie juridique doit être cette constatation que la plupart des rapports de droit n'accèdent pas à la litigiosité (...) le contentieux ne nous découvre ainsi qu'une très faible portion du droit effectif (...) le droit est infiniment plus grand que le contentieux" ${ }^{4}$.

On comprend donc que la régularité de la sanction ne soit pas un élément si important que 1 'enseignent les théories classiques ${ }^{5}$.

b) la sanction peut n'être qu'une menace: Hoebel a raison d'envisager cette éventualité, mais il faudrait la souligner beaucoup plus fortement. Nous verrons ${ }^{6}$ que, très souvent, les Inuit mettent en œuvre des modes de sanction psychologiques consistant, selon des modalités diverses, à faire comprendre à l'individu fautif qu'à moins d'amendement, il sera de plus en plus exclu de la communauté, depuis la mise à l'écart temporaire jusqu'au bannissement ou à la liquidation physique. Certains types de sanctions peuvent même consister en la seule menace de la

1 Cf. N. ROULAND, "Ethnologie juridique des Inuit: Approche bibliographique critique", Études/Inuit/Studies, vol. 11, n 1 (1978), 122

E. ADAMSON-HOEBEL, op. cit., 28.

Cf. les critiques de J. CARBONNIER, Flexible Droit, (Paris, 1971), 26 sur la définition de ADAMSON-HOEBEL.

Cf. J. CARBONNIER, op. cit., 18.

5 Ce qui ne signifie pas que la règle de droit ne revête pas un caractère obligatoire : cf. infra, pp. 18-19.

6 Cf. infra, pp. 37 sq. 
sanction : ainsi du meurtrier qui, dans certains cas de vendetta, va devoir, pendant plusieurs années, vivre dans l'attente de l'action de représailles ${ }^{1}$.

[p. 17]

c) la sanction se définit par l'application de la force physique : c'est certainement sur ce point que la définition de Hoebel pèche le plus : comme nous venons de le voir, la sanction peut très bien consister en des moyens de pression uniquement psychologiques ${ }^{2}$.

d) agent de la sanction : il n'est pas forcément extérieur à l'individu coupable : dans les sociétés Inuit, la pression du groupe tend, tout d'abord, à inciter le fautif à s'amender lui-même ${ }^{3}$.

Ces remarques ne paraissent pas superfétatoires : négliger ces corrections peut conduire à de graves erreurs d'appréciation, et même à des contre-sens. Nous en trouvons un exemple dans les conclusions de G. Van den Steenhoven, qui affirme d'ailleurs plusieurs fois son adhésion à la définition de Hoebel, même s'il y ajoute certains éléments ${ }^{4}$. Étudiant les Inuit du Caribou - sans doute la plus archaïque et la moins formalisée des populations Inuit - l'auteur constate qu'à une infraction donnée ne correspond pas forcément une sanction donnée, et que même cette sanction peut ne pas survenir. Il en déduit l'absence de droit chez cette population ${ }^{5}$. Or, il est certain que, si l'on néglige l'aspect psychologique de la sanction, le fait qu'elle peut se réduire à une simple menace, la souplesse d'adaptation aux cas concrets, et la conception particulière aux peuples archaïques de la fonction de la sanction ${ }^{6}$, on ne peut que parvenir à ce résultat. Alors qu'à notre sens, n'est absente, dans ces populations, que la forme sous laquelle s'exprime le droit dans nos sociétés, et non pas le phénomène juridique lui-même. Il s’agit donc bien là d'une vision très ethnocentriste. G. Van den Steenhoven note ainsi ${ }^{7}$ que le menteur, le voleur, le paresseux, celui qui manque à ses devoirs familiaux est mal vu par le groupe qui [p. 18] se désintéresse de lui et peut aller jusqu'à l'abandonner : mais le groupe peut choisir des moyens très divers pour faire comprendre au fautif qu'il est dans son tort. Dans cette variété des sanctions, G. Van den Steenhoven se refuse à voir des processus juridiques, qui ne peuvent, pour lui, consister que dans l'application d'une sanction codifiée à des fautes hiérarchisées. Cette position est beaucoup trop dogmatique et n'est que la conséquence d'une application rigide du critère de la sanction tel qu'il a été défini par la pensée occidentale, et qui est, tel

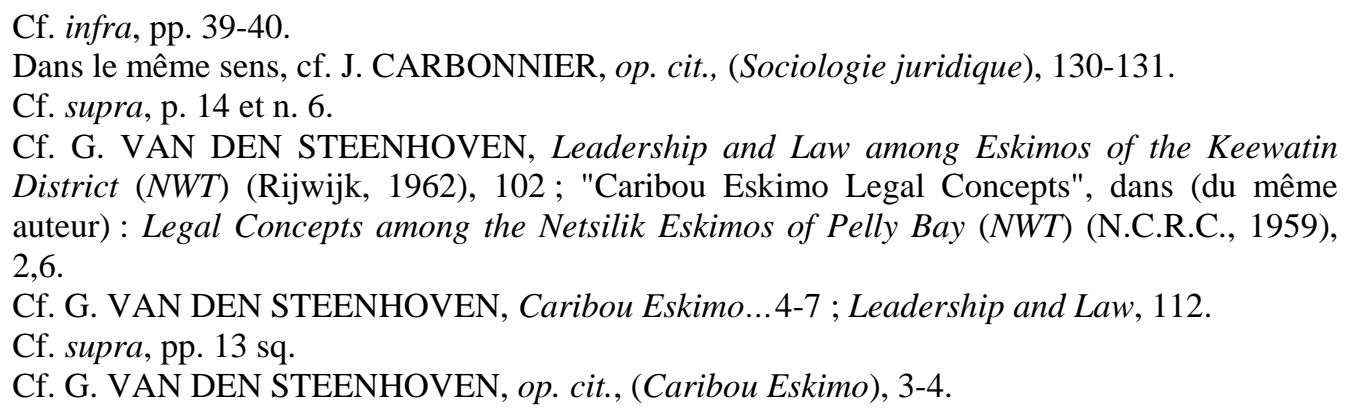


quel, intransposable dans le monde juridique des sociétés archaïques. D'ailleurs, l'auteur montre plus loin la même méconnaissance quant à la finalité de l'institution judiciaire dans ces sociétés : reprenant les conclusions de Birket-Smith - auquel on ne peut reprocher de n'avoir pas été juriste - G. Van den Steenhoven écrit que le groupe n'a pas vraiment de rôle judiciaire, mais qu'il intervient uniquement pour rétablir l'ordre ${ }^{1}$. Là encore, l'ethnocentrisme affleure : que le processus judiciaire chez les Inuit soit plus conçu en fonction du rétablissement de l'ordre public que du châtiment éventuel du coupable ${ }^{2}$ ne signifie nullement que la communauté n'intervienne pas par une action spécifiquement juridique et sous une forme judiciaire.

Il faut donc dire nettement que l'absence du recours à la force physique ainsi que celle de l'application automatique d'un système hiérarchisé de sanctions ne prouve en rien l'inexistence d'une structure juridique de la société en cause : la sanction peut être psychologique, différée, être appliquée par le fautif lui-même, et revêtir des formes adaptées aux nécessités concrètes.

En réalité, ces remarques nous conduisent à une nouvelle définition de la sanction du droit, plus adaptée aux sociétés archaiques. Isolons-en les éléments.

J. Poirier a fort bien montré que la sanction devait posséder au moins deux caractères: la prédétermination, le caractère obligatoire ${ }^{3}$. [p. 19] La prédétermination ne consiste pas, comme le croit G. Van den Steenhoven, dans la précision nettement exprimée de telle ou telle sanction : Il suffit que le contrevenant sache d'avance que tel comportement provoquera une réaction de la part du groupe social. Ainsi, pour reprendre l'exemple des Inuit du Caribou, le menteur sait bien qu'il s'expose à la moquerie, ou à des formes d'ostracisme plus ou moins mitigées : le fait qu'il ne sache pas forcément de quelle, modalité précise usera le groupe pour le sanctionner ne signifie pas qu'il n'y ait pas obligation juridique. D'autre part, la sanction doit être obligatoire, s'imposer au juge et aux parties : là encore, cela ne signifie pas qu'elle doive se manifester automatiquement sous une forme concrète, par l'emploi de la force physique, et systématique. Sinon, on commettra l'erreur de G. Van den Steenhoven en concluant que la sanction est irrégulière, et que donc le droit est absent : la mise en jeu de sanctions psychologiques n'entame en rien la réalité de la sanction, et donc son caractère obligatoire, et par là l'existence d'un système juridique.

Ces quelques remarques montrent bien qu'il faut élargir la notion de sanction si on veut continuer à lui faire tenir la place qui lui revient dans le repérage du phénomène juridique. Un comportement, une matière, sont juridiques sans qu'il soit besoin qu'ils reçoivent une sanction effective : sinon le droit se confondrait avec son contentieux. En ce sens, la sanction n'est donc qu'un critère partiel, c'est-

Ibid., 7.

Cf. supra, p. 13.

Cf. J. POIRIER, op. cit., 1092-1094. L'auteur y ajoute la légitimité, mais cela ne concerne pas nos sociétés Inuit, où l'apparition d'une organisation politique différenciée est très rare. 
à-dire qu'elle n'en est plus un... Ce qui compte est l'éventualité de la sanction, la possibilité de sa mise en jeu : les normes sociales deviennent juridiques dans la mesure où elles peuvent donner lieu a un jugement : ainsi est intégré tout l'aspect psychologique de la sanction, si important dans les sociétés archaïques, particulièrement inuit. Ce jugement ne doit pas être forcément conçu comme très formalisé : il peut émaner, de façon plus ou moins diffuse, de l'opinion publique, et non d'un "tribunal" et s'exprimer sous des formes très nuancées. On ne peut ici que citer les lumineuses analyses de J. Carbonnier :

"Ce qui est propre au droit, c'est une mise en question organisée, une institution de contestation : (...) c'est la forme typique du procès, avec son issue dans le jugement. Procès et jugement sont des phénomènes psycho-sociologiques si [p. 20] irréductibles à tous autres et si spéciaux au droit qu'il paraît rationnel d'en faire l'indicatif de la juridicité (...). C'est la seule éventualité du jugement, l'eventus iudicii, qui est la justiciabilité, non pas le jugement effectif, encore moins la condamnation (...) le jugement est un doute qui décide ; le procès, l'institution d'une mise en doute avec une décision au bout. Dès qu'une relation entre deux personnes peut faire l'objet d'un débat devant une personne tierce qui tranchera, on doit conclure qu'elle n'appartient plus au domaine des mœurs, mais est entrée dans le royaume incertain du droit" $^{1}$.

Dans le cas des sociétés archaïques a-étatiques, telles que les sociétés Inuit, on devra évidemment toujours considérer que le consensus, comme la réprobation, l'eventus iudicii ou le jugement lui-même revêtent souvent un caractère diffus, mais non moins réel pour autant ${ }^{2}$ : les sociétés Inuit sont des sociétés de refoulement ${ }^{3}$.

Nous nous en apercevrons constamment en étudiant les modes psychologiques de sanction chez certaines populations Inuit.

J. CARBONNIER, op. cit., (Sociologie juridique), 135-136.

Cf. la conclusion de l'article de C.C. HUGHES, "Anomie, the Ammassalik, and the standardization of error", dans South western Journal of Anthropology, XIV-4 (1958), 375, qui critique, à juste titre, la vision très ethnocentriste de J. MINSKY sur la société Inuit d'Ammassalik qu'elle estime anarchique. C.C. HUGHES cite à bon droit R.M. WILLIAMS Jr. (American Society (New York, 1951), 17) : "Cultural regulation occurs where normative standards are maintained by the diffuse action of the whole social group (...). Sanctions are meted out through diffuse consensus ; the mechanisms of enforcement of norms are nonnucleated ; no particular individual or group is clearly responsible for maintaining the accepted patterns".

3 Cf. l'interview de J. MALAURIE à l'ORTF (1974) (Émission Radioscopie). 


\section{Section II : $\quad$ LES MODES SOCIO-PSYCHOLOGIQUES DE SANCTION : PRÉ-ÉMINENCE DU GROUPE}

\section{Retour à la table des matières}

"...la loi du groupe prédomine, et l'individu n'existe que dans la mesure où il se relie au groupe et fonctionne dans le groupe (...). Dans cette société, on n'est jamais aidé, et l'on dot aider le groupe. Jamais l'individu ne s'exprime : jamais il ne dira "je pense", mais "on pense" ou "le groupe pense" ${ }^{1}$.

\section{[p. 21]}

L'organisation économique et socio-politique des sociétés inuit, avec des diversités dues aux contextes locaux, repose en effet sur la loi de primauté du groupe sur l'individu : l'individu doit s'effacer devant la collectivité, mais sans elle il est psychologiquement et matériellement incapable de survivre. Au niveau de la sanction du droit, ce principe sociologique a pour effet que l'opinion publique joue un rôle prépondérant dans l'application des procédures destinées à rectifier un comportement déviant. Exprimant le jugement du groupe - que certains maïeutes peuvent aider à se dégager ${ }^{2}$ - elle est souveraine.

La communauté, à des degrés divers, intervient, en effet, dans pratiquement toutes les procédures de sanctions. Dans certains cas, elle le fera de façon non formaliste, par pressions et incitations d'ordre psycho-sociologique : le comportement fautif sera traité par une thérapeutique mettant en jeu la dérision, la réprimande, certaines injures, la confession, etc... Dans d'autres cas, le groupe interviendra selon des processus institutionnels : limitation des vendettas, action publique (essentiellement la liquidation physique du perturbateur et les duels judiciaires).

Cette intervention de la communauté n'est nullement anarchique, mais au contraire réglée : comme dans toutes les sociétés archaïques, on cherchera à se référer à la coutume. Le groupe a donc besoin de maïeutes, de Sages, qui connais-

1 Ibid. Dans le même sens : "... seul le groupe permet à l'homme de vivre. L'homme ainsi protégé, réserve, en retour, à la société, sa ... force et sa pensée. Au point que, refoulant toute réaction individuelle, il ne s'explique qu'à la troisième personne, se coulant dans une pensée générale, celle du groupe. Jamais un Esquimau ne dit : je pense, mais les "Inuit" pensent (J. MALAURIE, "Raids et esclavage dans les sociétés autochtones du détroit de Behring", dans InterNord, 13-14 (déc. 1974), 7).

2 Cf. infra, pp. 22-29. 
sent les coutumes et peuvent soumettre leur interprétation à la communauté en même temps qu'ils aident - ou contraignent - le coupable à reconnaître ses fautes et à s'amender.

Compte tenu de ces remarques, nous étudierons successivement les problèmes suivants ; le rôle des maïeutes, les types de procédures de sanction du droit sociopsychologiques (nous étudierons les modes institutionnels d'intervention du groupe dans le second chapitre de cette étude).

[p. 22]

\section{A) LE RÔLE DES MAÏEUTES ET LA STRUCTURE COUTUMIÈRE DU DROIT INUIT}

\section{a) Prééminence de la coutume}

\section{$\underline{\text { Retour à la table des matières }}$}

C'est à dessein que nous utilisons ici le terme de maïeutes, dans son acception quasi-socratique : le maïeute est celui qui va accoucher l'esprit du fautif, l'aider à se rendre compte de ses torts et à les amender. Ces maïeutes sont les personnalités du groupe : shaman, sages, umialik, leader de la famille ou du village. Comme nous le signalions plus haut, leur rôle ne peut bien se comprendre que si l'on garde en mémoire ${ }^{1}$ que la structure du droit inuit est essentiellement coutumière. Dans une société a-étatique, le droit ne peut pratiquement naître que de l'opinio communis et de la répétition de précédents (tout le droit européen du Haut MoyenÂge en témoigne) ${ }^{2}$. Outre qu'elle est le seul mode possible de formation du droit dans ce contexte, la coutume répond au besoin de protection éprouvé par l'individu dans la relative fixité qu'elle apporte. La règle coutumière représente ce qui a victorieusement subi l'épreuve du temps et résisté aux forces d'agression développées par l'environnement contre le groupe. La coutume n'est donc pas un comportement "biologique", instinctif, comme le voudraient certains, mais au contraire le fruit d'une réflexion empirique dominée par la crainte de ce par quoi l'individu estime pouvoir être dépassé. La coutume n'en est pas pour autant un droit intangible : avec la souplesse d'adaptation aux circonstances concrètes qui les caractérise, les Inuit savent modifier leurs coutumes quand l'évolution des conditions historiques les fait apparaître comme dépassées. À titre d'exemple, J. Malaurie a montré que les Inuit Polaires de Thulé (Nord-Ouest Groënland) avaient modifié leurs

Cf. N. ROULAND, op. cit., (Approche du phénomène juridique...), 58, 61-64.

Sur les caractères sociologiques et juridiques de la coutume dans les sociétés féodales médiévales européennes, cf. R. MASPETIOL, op. cit., 164-171. 
coutumes économiques et démographiques en fonction des variations de climat ${ }^{1}$ : de 1600 à 1800, [p. 23] le premier petit âge de glace provoque un refroidissement qui fait prôner par les shaman le malthusianisme démographique et l'austérité économique ; les Inuit doivent, pour survivre, se restreindre à la consommation des oiseaux, abandonner le kayak, et ne chasser le phoque que de façon occasionnelle ; les interdits de mariage proscrivent l'union entre cousins jusqu'au $6^{\mathrm{e}}$ degré : dans un groupe de dimensions restreintes et fort éprouvé par la perte en effectifs humains due à la famine, une réglementation plus lâche, en permettant un taux de consanguinité plus élevé (qui, grâce à ces mesures, demeura exceptionnellement faible : entre 0,0002 et 0,0003 ) aurait conduit le groupe à sa perte. Mais, à partir de 1862-66, le climat se réchauffe de nouveau, le gibier devient plus abondant et permet au groupe d'augmenter sa taille : les Inuit de Thulé reprennent la chasse aux cétacés de leurs aïeux, et en une génération - de 1860 à 1895 - la population triple : on peut supposer avec J. Malaurie que, pour mettre en œuvre cette véritable planification démographique, le groupe sut utiliser toute une série de moyens : accroissement de la liberté génésique, proscription de l'infanticide, avance de l'âge de la puberté, etc.

On objectera que les coutumes de chasse n'ont pas de caractère juridique : cela pourrait encore se discuter, dans la mesure où les [p. 24] interdits de chasse sont sanctionnés par le groupe. Mais, en tout cas, les prohibitions des unions considérées comme incestueuses sont bien de nature juridique.

Certains auteurs, comme G. Van den Steenhoven, ont nié que le droit puisse être coutumier ${ }^{2}$ : pour G. Van den Steenhoven, un comportement spontané ne peut être juridique : la coutume n'est donc pas source du droit. Il est vrai que toute coutume n'est pas juridique : pour distinguer les juridiques des non juridiques, il n'est besoin que de recourir au critère de l'eventus iudicii ${ }^{3}$. Mais il est bien certain que la coutume peut être source du droit : cette idée a toujours été acceptée

1 Cf. J. MALAURIE, op. cit. (Les civilisations esquimaudes), 57-59; Les derniers rois de Thulé (Paris, 1975), 88, 92 ; "Pour une préhistoire des sociétés maritimes et de la navigation préesquimaude dans les zones côtières du Pacifique Nord, des mers de Tchoutchi et de Beaufort", dans Inter-Nord, 12 (1972), 175-177 ; "Spécificité des sociétés esquimaudes", dans Bulletin de la Société Royale de Géographie d'Anvers, LXXV-1-2 (1963), 10 ; J. MALAURIE, L. TABAH, J. SUTTER, "L'isolat esquimau de Thulé", dans Population (oct.-déc. 1952), 675-691. Dans le même sens de l'influence des changements de climats sur l'histoire sociale des peuples arctiques, cf. J. MALAURIE, Y. VASARI, H. HYVARINEN, G. DELIBRIAS, J. LABEYRIE, "Preliminary Remarks on Holocene Paleo-climates in the regions of Thule and Inglefield Land, above all since the beginning of our own era", University of Oulu. Acta Universitatis Ouluensis : Climatic changes in Arctic Areas during the last Ten-Thousand Years, (Oulou, 1972), 105-136 ; J. MALAURIE, "Les changements de climats dans le N.O. du Groënland pendant le post-glaciaire récent : nouvelles conclusions polynologiques, géomorphologiques et ethnogénétiques", dans Les Vikings (sous la dir. de R. BOYER), Bibliothèque arctique et antarctique, V (1974), 89-101. Les historiens commencent à manifester de l'intérêt pour l'histoire du climat : cf. E. LE ROY LADURIE, Histoire du climat depuis l'an mil (Paris, 1967).

Cf. G. VAN DEN STEENHOVEN, op. cit., (Caribou Eskimo...), 1.

Cf. supra, pp. 19-20. 
par les juristes les plus pointilleux eux-mêmes ${ }^{1}$, ainsi que par les anthropologues ${ }^{2}$.

Les Inuit expriment d'ailleurs très bien eux-mêmes la force contraignante de la coutume :

[p. 25]

- Nelson rapporte des Inuit de l'Alaska " ... si vous leur demandez pourquoi ils font telle ou telle chose, ils vous répondent invariablement : "nous avons toujours fait ainsi" ${ }^{3}$.

- Un Inuk Iglulik répondit un jour à K. Rasmussen qui le pressait de questions : "Trop penser ne fait qu'amener de la perturbation... Nous, les Inuit, nous ne prétendons pas donner de solution à toutes les énigmes. Nous répétons les vieilles histoires telles qu'on nous les a racontées, et avec les mots dont nous nous souvenons... Vous voulez toujours que les histoires surnaturelles aient un sens, mais cela nous est égal. Nous sommes contents de ne pas comprendre" ${ }^{4}$.

Le scepticisme à l'égard de la tradition demeure exceptionnel, mais peut parfois se manifester : un Inuk du groupe Ukuhikjalingmiut dit à K. Rasmussen qu'à son avis toutes les traditions n'étaient pas bonnes à croire, car la mémoire de ceux qui les transmettaient - nos maïeutes - n'était pas toujours fidèle : les shamans eux-mêmes ne connaissaient pas toujours le fondement des règles qu'ils appliquaient et auxquelles ils croyaient ${ }^{5}$.

1 "Les règles de droit qui régissent les hommes dans leurs rapports sociaux proviennent de deux sources diverses : de la loi et de la coutume. La loi est une règle de droit conçue, formulée et édictée par une autorité spécialement chargée de cette mission dans la société, autorité qui exerce le pouvoir législatif. La coutume, au contraire, est une règle de droit qui se dégage lentement des faits, des pratiques habituellement suivies dans le milieu social. Elle se forme spontanément et devient obligatoire sans avoir reçu de consécration expresse des pouvoirs établis. Lorsque les membres d'une société ont, pendant longtemps, observé, dans leurs rapports juridiques, les mêmes façons d'agir, ces façons d'agir deviennent des modèles. Elles sont considérées comme des règles-types auxquelles tous ceux qui se retrouveront plus tard dans la même situation seront tenus de se conformer. La loi et la coutume présentent ce caractère commun d'engendrer le droit positif qui gouverne les sociétés, mais elles procèdent de façon différente : la règle légale naît d'un acte volontaire et précis du pouvoir législatif ; la règle coutumière se forme peu à peu dans le milieu même qu'elle doit régir (...) À l'origine des sociétés, la coutume est la source presque unique du droit" (A. LEBRUN, La Coutume (Thèse Droit Caen, 1932).

2 "By customary law, I mean a law that is internalized by a social group. We call a law internalized when the majority of the membership of the group consider it to be binding, as when it stands for the "only proper behaviour in a given situation" (L. POSPISIL, "The Nature of Law", dans Transactions of the New York Academy of Sciences, Ser. II, XVIII-8 (June 1956) 753)

3 Cité par H. KONIG, "Der Rechtsbruch und sein Ausgleich bei den Eskimo", dans Anthropos, XVIII-XIX, 1-2-3 (1923-24), 492.

4 Cit. par E. ADAMSON-HOEBEL, op. cit., 68-69. Sur la valorisation du droit par le mythe, cf. M. ALLIOT, "L'acculturation juridique", dans Ethnologie Générale, op. cit., 1181-1184.

5 Cf. K. RASMUSSEN, The Netsilik Eskimos. Social life and spiritual culture, Rep. of the 5th Thule Expedition, 1921-24, VII-1/2 (Copenhagen, 1931), 502. 
Compte tenu de la nature coutumière du droit inuit, dont les lignes qui précèdent ont eu pour objet de souligner l'importance, on comprend mieux la nécessité des maïeutes, et la nature de leur rôle. Ces personnages, que nous allons énumérer, sont nécessaires en tant que véhicules et interprètes de la tradition : tous les individus n'ont pas une connaissance parfaite des coutumes, et le recours à des personnalités dominantes est nécessaire dans les cas où la coutume à appliquer et la solution de droit ne sont pas évidentes. Mais ces maïeutes n'en deviennent pas pour autant des juges : stricto sensu, on ne peut les qualifier d'autorités judiciaires : ils aident à clarifier les données et incitent le coupable à reconnaître ses fautes, mais c'est l'opinion [p. 26] publique qui tranche ${ }^{1}$. Étudions maintenant de plus près l'identité de ces maïeutes.

\section{b) Les principaux maïeutes}

\section{$\underline{\text { Retour à la table des matières }}$}

Dans tout groupe Inuit, il y a dualité entre l'autorité civile et religieuse ${ }^{2}$ : le shaman, par sa connaissance des tabous, est le véhicule de la tradition religieuse et peut être à l'origine de prises de sanction par le groupe. À côté de lui, on trouve des leaders civils, au niveau de la famille ou du village. Dans les sociétés alaskiennes, où l'organisation politique est plus différenciée qu'ailleurs, l'umialik joue un rôle important. Nous étudierons donc successivement les rôles des shamans et des leaders civils.

\section{1) Le shaman}

Au terme du récit de ses séjours chez les Inuit de l'Alaska, V. Stefansson rapporte que, parmi eux, l'homme sage est celui qui connaît un grand nombre de tabous, et que le prestige appartient à celui qui en sait le plus sur tout ce qu'il convient de ne pas faire ${ }^{3}$.

Il ne faut pas en déduire pour autant - et la constatation s'applique à l'ensemble des sociétés inuit - que le shaman a un pouvoir créateur du droit ${ }^{4}$ : politiquement il n'est pas un "chef", et juridiquement la source du droit est la coutume, donc le groupe : le shaman n'intervient que pour la présenter et la faire appliquer. Nonobstant ce principe fondamental, le shaman tire socialement de ce rôle un important pouvoir : chez les Nunamiut, l'autorité civile, l'umialik, ne craint que le pouvoir du shaman ${ }^{5}$. On comprend d'autant mieux la révérence dont il [p. 27] est entouré, quant aux pouvoirs dont il dispose dans son domaine spécifique, que nous sommes là en présence d'un trait spécifique de la mentalité archaïque : la

\footnotetext{
Dans le même sens, cf. J. MALAURIE, op. cit., (Raids et esclavage ...), 7.

Cf. J. MALAURIE, ibid.

Cf. V. STEFANSSON, My Life with the Eskimo (New York, 1922), 412.

Cf. H. KUNIG, op. cit., 492.

Cf. L. POSPISIL, "Law and societal structure among the Nunamiut Eskimo", dans Explorations in cultural anthropology (Mac Graw Hill, 1964), 398.
} 
violation d'un tabou conduit immanquablement, dans l'esprit des Inuit, à des catastrophes d'ordre naturel : maladie ${ }^{1}$, famine, fuite du gibier, etc. Ce trait est celui de l'homogénéité de la Nature et de la Société : les sociétés archaïques établissent le plus souvent une liaison intrinsèque entre les événements proprement sociaux et les événements naturels ; nature et société obéissent à des règles communes : l'ordre social troublé, une perturbation correspondante doit apparaître dans l'ordre naturel ${ }^{2}$. La distinction posée par les Grecs entre nomos et physis est inconnue. Le rôle du shaman est donc de rétablir l'harmonie passagèrement troublée entre ces deux univers. Il est donc attesté en des termes souvent identiques dans de très nombreuses sociétés inuit. Donnons-en quelques exemples :

- chez les Inuit Polaires (Nord-Ouest Groënland), lorsque le groupe se trouve dans l'incertitude quant à la solution à adopter, l'angakok est consulté ${ }^{3}$.

- sur la côte Ouest du Groënland, l'angakok peut faire condamner par la communauté les individus dénués d'un réseau de protections parentales : essentiellement les veuves sans protecteurs et les couples sans enfants ${ }^{4}$.

- au Labrador, l'angakok frappe le coupable d'interdits religieux qui le contraignent à la fuite ${ }^{5}$.

- à Ammassalik (côte Est GroënIand), l'angakok est l'interprète des règles de droit et les met en œuvre ${ }^{6}$.

[p. 28]

- chez les Inuit Iglulik, le rôle judiciaire du shaman est également attesté ${ }^{7}$.

- chez les Inuit du Cuivre, en cas de violation de tabou, le shaman doit découvrir le coupable et dire ce qu'il faut faire pour rétablir l'ordre ${ }^{8}$.

- chez les Inuit du Caribou, le shaman, à côté du chef de famille, du voyant, intervient aussi dans le processus judiciaire. Il le fait, notamment, en dirigeant la confession publique du coupable qui, s'il reconnaît ses fautes, est en principe absout ${ }^{9}$.

\footnotetext{
Ainsi les Inuit Polaires analysent-ils la maladie à la fois en termes socio-juridiques, religieux et biologiques : la maladie est toujours la conséquence de la violation d'un tabou par le malade (Cf. Erik HOLTVED, "Contributions to Polar Eskimo Ethnography" (M.o.G., Bd. 182, no 2 Copenhagen, 1967), 151.

Cf. R. PINTO et M. GRAWITZ, Méthodes des Sciences Sociales (Paris, 1969), 1-2.

Cf. H.P. STEENSBY, op. cit., 369-370.

Cf. H. KONIG, op. cit., 306.

Ibid.

Cf. C.C. HUGHES, op. cit., 371.

Cf. T. MATHIASSEN, Material Culture of the Iglulik Eskimos, Rep. of the 5th Thule Expedition 1921-24, VI-I (Copenhagen, 1928), 209.

Cf. D. PRYDE, Nunaga (Paris, 1974), 158.

Cf. J. GABUS, Vie et Coutumes des Esquimaux Caribou (Lausanne, 1944), 139.
} 
Cette pratique de la confession publique dirigée par le shaman, est également attestée sur la côte ouest du Groënland, mais semble inconnue sur la côte Est ${ }^{1}$. Elle se déroule de la façon suivante ${ }^{2}$ chez les Inuit Iglulik : la communauté réunie autour du suspect, le shaman dirige l'enquête en posant des questions au fautif, tout en dansant autour de lui. La communauté joue elle aussi un rôle actif car, sans accabler l'individu en cause, elle l'exhorte par des chants à se laver de sa faute et obtenir ainsi le pardon. Le coupable, petit à petit, avoue que le mal dont il souffre est dû à la violation d'un tabou qu'il a commise. Parfois la communauté complète ses aveux (dans des groupes restreints, les faits sont facilement connus de tous), en même temps qu'elle exhorte le shaman à absoudre le coupable. Celuici peut, cependant, estimer que la confession en elle-même lave le coupable de sa faute, ou énoncer certaines sanctions : abstention de certaines nourritures, obligation pour une femme d'avoir des relations sexuelles avec lui, obligation faite à un couple de se séparer, échange de conjoints ${ }^{3}$, etc.

On soulignera essentiellement deux points :

[p. 29]

- la communauté joue un rôle aussi actif que le shaman et est, en quelque sorte, l'intermédiaire entre lui et le coupable, puisqu'elle s'adresse aussi bien à l'un qu'à l'autre

- dans de nombreux cas, la sanction ne consiste pas dans l'application de mesures au fautif, mais dans la confession elle-même ${ }^{4}$, qui est censée l'absoudre de sa faute. Les mesures qui peuvent éventuellement suivre ont un caractère objectif : elles tendent beaucoup plus au rétablissement de l'ordre qu'au châtiment du coupable.

Ibid., 142.

Cf. E. ADAMSON-HOEBEL, op. cit., 71, n. 4.

Ibid., 71-74.

C'est le cas chez les Inuit Kangirsujjuaaq (Nouveau-Québec) (Cf. B. SALADIN D'ANGLURE, L'organisation sociale traditionnelle des Esquimaux de Kangirsujjuaaq (Québec, 1967), 155.

On retrouve - et personne ne s'en étonnera - des pratiques semblables dans les groupements humains et sociétés à tendance collectiviste : confession publique dans les communautés monacales ; pratique dite de l'Épreuve' dans les prisons de la Chine communiste : celui qui a commis une infraction au règlement de la prison ou s'est rendu coupable de déviation idéologique est soumis aux exhortations très pressantes de ses camarades de cellule (dirigés par un chef de cellule) à avouer ses fautes, après quoi on considère qu'il a fait le pas le plus important dans la voie de l'amendement. Souvent cette confession s'accompagne d'une absence de sanction corollaire (Cf. J. PASQUALINI, Prisonnier de Mao). L'auto-critique est d'ailleurs une pratique très répandue dans le monde communiste. 
On saisit donc par là tout le côté psychologique de la sanction, qui est bien réelle, mais à aucun moment n'a mis en jeu l'application de la force physique ${ }^{1}$.

\section{2) Leaders civils}

À l'intérieur de la famille, le père de famille peut décider de la sanction à appliquer: ainsi de l'ihumatar familial chez les Inuit Netsilik de Pelly-Bay (T.N.O.) ${ }^{2}$; également chez les Caribou ${ }^{3}$. Il semble d'ailleurs que ce principe soit d'application générale ${ }^{4}$.

[p. 30]

Si l'on se situe au niveau de la société globale, de nouveaux maïeutes apparaissent : les Vieux, les leaders "politiques".

- les Vieux : étant donné leur âge, on pense que c'est eux qui ont le plus d'expérience et qui se souviennent le mieux des anciennes coutumes. Leur intervention est attestée chez les Nunamiut (Alaska) ${ }^{5}$ : quand un différend n'a pu être réglé dans le cadre purement familial, il est porté devant la communauté, dans la Grande Maison (karigi). Les individus les plus âgés se mettent alors à parler et expliquent comment, dans le passé, des cas semblables ont été réglés. On voit qu'une fois de plus, c'est à la coutume qu'on fait appel : les Vieux ne disent pas le droit, ils le transmettent. Ils n'ont donc, pas plus que le shaman, de rôle créateur du droit. Ce ne sont pas non plus des juges, car c'est aux hommes du karigi, à la communauté, de décider des sanctions éventuelles. Cependant, les Vieux jouent un rôle actif : non seulement ils transmettent la coutume, mais ils exhortent le récalcitrant à reconnaître ses fautes. Leur intervention dénote déjà que le conflit revêt une certaine gravité, car on fait tout pour éviter que les différends ne montent jusqu'à l'échelon de la réunion dans le karigi. Quand un tel fait se produit,

1 Sur la confession publique chez les Inuit, cf. également : N.H.H. GRABURN, "Eskimo law in light of self and group interest", dans Law and society Review, IV-I (1969), 49.

Cf. G. VAN DEN STEENHOVEN, op. cit., (Legal Concepts), 36.

Cf. J. GABUS, op. cit., 139.

4 Cf. H. KUNIG, op. cit., 300 sq ; J. MALAURIE, op. cit. (Les civilisations esquimaudes). À Ammassalik, il semble également appartenir aux aînés de la famille (Cf. C.C. HUGHES, op. cit, 371).

5 Cf. Wendell H. OSWALT, Alaskan Eskimos (San Francisco, 1967), 178 ; N.J. GUBSER, The Nunamiut Eskimos. Hunters of Caribou (London, 1969), 186. Dans la région comprise entre le bas Yukon et le Kuskokwim, ces "anciens" sont nommés naskok (tête); chez les Unalit, anaiyukok (celui qui entend tout). Mais ces termes sont aussi appliqués aux individus qui ont acquis une position de leadership grâce à leur richesse (cf. L. FAINBERG, "On the question of the Eskimo kinship system", dans Arctic Anthropology, IV-1 (1967), 248. 
cela suppose que toute une série de moyens pour résoudre le conflit a été déjà utilisée, mais s'est révélée inopérante ${ }^{1}$;

[p. 31]

- leaders "politiques" : on sait qu'un individu, pour être reconnu comme le leader du groupe, doit constamment faire preuve d'un ensemble de qualités : succès à la chasse, supériorité physique, bon caractère, générosité, etc. On attend également de lui qu'il assure la transmission et l'interprétation de la Tradition, qui est l'ensemble des conduites sociales - juridiques et non juridiques - permettant la survie. En baie de Foxe (Canada), ce personnage est l'issoumatar (celui qui pense beaucoup) ; à Thulé (Nord-Ouest Groënland), c'est le nalaqaq (le terme indique plus l'autorité que la sagesse) ${ }^{2}$.

Dans les sociétés inuit où la structure du commandement est plus nettement affirmée qu'ailleurs, le leader joue un rôle très important dans la détermination de la coutume applicable et la procédure de sanction. Ainsi, chez les Inuit Chugach (Sud-Est Alaska), le chef du village peut convoquer le personnage jugé dangereux, lui faire des reproches devant la communauté, et le menacer d'un ostracisme temporaire ${ }^{3}$. Dans les autres sociétés alaskiennes, le rôle de l'umialik est également très significatif. L’umialik est un personnage considérable : propriétaire d'un umiak, l'embarcation qui permet la chasse à la baleine, il bénéficie de la supériorité économique et dirige les opérations de chasse ${ }^{4}$. Il est aussi un leader "politique", bien que son autorité, à l'instar de celle de tous les leaders inuit ${ }^{5}$, soit essen-

1 On notera à cette occasion une certaine similitude avec la justice africaine : "C'est donc seulement quand le contrôle social, c'est-à-dire l'équilibre des structures fondamentales est menacé que l'individu se trouve être justiciable. En outre, le recours à la justice est considéré comme une chose à éviter au maximum. Il y a en effet des risques de conflits insolubles. C'est pourquoi la procédure judiciaire est d'abord précédée de mises en garde, de pressions sociales, prévenant l'individu sur la conséquence d'actes éventuels. L'individu est ainsi mis en face de ses responsabilités et des exigences du contrôle social. S'il persévère dans la conduite, le mécanisme judiciaire est alors mis en marche". (E. LE ROY, Introduction aux institutions politiques africaines, Paris I, Séminaire d'anthropologie politique, document pédagogique no 2, 150).

2 Cf. J. MALAURIE, op. cit., (Les derniers rois ...), 150 ; op. cit. (Les civilisations esquimaudes...), 82.

3 Cf. K. BIRKET-SMITH, The Chugach Eskimo (Copenhagen 1953), 98.

4 Cf. par exemple, le rôle de l'umialik, chez les Tareumiut : cf. W.H. OSWALT, op. cit., 180181. (Cf. aussi N.J. GUBSER, op. cit., 180-184).

5 Cf. J. MALAURIE, op. cit. (Les Civilisations Esquimaudes...), 83. Ainsi la notion de leadership est-elle toujours subordonnée à celle d'intérêt public : "Non seulement sa générosité (celle du leader), le caractère égalitaire de la répartition des prises rendent impossible toute thésaurisation individuelle, toute différenciation par la richesse ; mais c'est surtout par les dons que ce chef sanctionne la nature quasi-délégataire de sa puissance et restitue, en fait, à la collectivité les biens qu'il n'a pu et su accumuler qu'au prorata des moyens d'action qu'elle lui a consentis. À lui, le prestige et l'autorité. À chacun et en parts égales, les bénéfices". (J. MALAURIE, ibid.). 
tiellement précaire, car toujours susceptible d'être remise en question par le groupe.

L. Fainberg note que, dans le Nord-Ouest de l'Alaska, en 1944, c'était une assemblée du village, comprenant les hommes et les femmes, [p. 32] qui décidait des questions importantes : mais, au cours de ces assemblées, c'était pratiquement l'umialik qui "faisait" la décision ${ }^{1}$. Au niveau plus proprement judiciaire, celui de la résolution des conflits internes au groupe, nous possédons un certain nombre de données sur la fonction de l'umialik chez les Inuit Nunamiut, qui sont des chasseurs de caribou, et vivent dans le Nord de l'Alaska. Le terme d'umialik est donc ici dévié par rapport à sa signification première : il ne désigne plus, comme chez les populations alaskiennes côtières, le propriétaire d'un umiak. Mais il qualifie toujours la fonction du leader, qui est avant tout un bon et riche chasseur. Deux fois par an, lors des migrations saisonnières des troupeaux de caribous, les groupes Nunamiut, jusqu'alors dispersés, s'agglomèrent en bandes dirigées par les umialiks (chaque bande possède au moins un umialik ${ }^{2}$ ). Il doit aussi sa position prééminente à sa richesse, que sa famille l'a aidé à accumuler ${ }^{3}$, mais il doit se montrer très généreux ${ }^{4}$. Son seul rival possible est le shaman ${ }^{5}$. À côté de ses fonctions d'organisateur des expéditions de chasse et de guerre (contre les Indiens) ainsi que des cérémonies de la communauté ${ }^{6}$, l'umialik joue un rôle judiciaire : il juge les querelles internes ${ }^{7}$, et doit pour cela avoir une connaissance étendue du droit coutumier ${ }^{8}$.

La compétence judiciaire de l'umialik chez les Nunamiut n'est pas générale, mais limitée à des matières d'une certaine nature, en général celles dont la portée concerne, non seulement tel ou tel individu ou un groupe familial donné, mais surtout au premier chef, les perturbations graves de l'ordre public, les infractions aux règles de chasse, les délits à caractère économique (vols, atteintes aux biens) ${ }^{9}$. À un échelon [p. 33] inférieur, l’umialik peut connaître des causes de conflit internes à la famille (divorce, adultère, droit de correction sur les enfants), mais seulement dans la mesure où les autorités familiales n'ont pu, dans un premier stade, apporter de solution valables ${ }^{10}$. Ces distinctions ratione materiae sont fort intéressantes :

\footnotetext{
Cf. L. FAINBERG, op. cit., 248.

Cf. L. POSPISIL, op. cit., (Law and societal structure), 397 ; "Kinship terminology and kindred among the Nunamiut Eskimo", dans Ethnology, 1-2 (Ap. 1963), 191.

Cf. L. POSPISIL, op. cit., (Kinship terminology), 187-188.

Cf. supra, p. 26 ; L. POSPISIL, ibid., 181.

Cf. L. POSPISIL, op. cit, (Law and societal), 358.

Cf. supra, p. 31.

Cf. L. POSPISIL, op. cit., (Law and societal), 397.

Cf. L. POSPISIL, op. cit., (Kinship terminology), 181.

Cf. L. POSPISIL, op. cit., (Law and societal), 426.

Ibid. Son rôle s'inscrit donc à ce niveau dans le même cadre que celui des Vieux : cf. supra, p. 30 et n. 1.
} 
- elles révèlent un rapport entre la structuration juridique et la structure sociale de la société considérée ${ }^{1}$ : à chaque niveau sociologique correspond un système juridique :

à la famille appartiennent les différends d'ordre interne, qui sont réglés par les leaders de la famille ; à l'umialik appartient tout ce qui peut perturber l'équilibre de l'ensemble du groupe :

- $\quad$ soit les comportements réagissant directement sur la vie du groupe : le criminel récidiviste, par la répétition d'actes graves et imprévisibles atteint, non seulement ses victimes, mais également la collectivité, qui ne peut supporter ni l'affaiblissement en potentiel humain, ni la menace qui pèse sur chaque membre du groupe. De même, la chasse aux caribous étant collective, toute infraction à ses règles affecte immédiatement la vie de l'ensemble de la communauté ;

- $\quad$ soit les comportements réagissant indirectement sur la vie du groupe : un conflit non réglé à l'échelon inférieur perturbera les niveaux supérieurs. Ainsi l'adultère peut entraîner une extension des conflits par le système de la vendetta : la préservation de l'intérêt public exigera l'intervention de l'umialik pour empêcher cet enchaînement néfaste.

On constatera, d'ailleurs, que l'échelle des sanctions correspond à celle des systèmes juridiques, leur degré de gravité allant croissant au fur et à mesure qu'on passe de la sauvegarde d'intérêts privés à celle de l'intérêt public. Ainsi, les formes relativement "douces" d'ostracisme (mise à l'écart temporaire) sont décidées par le chef de la [p. 34] famille étendue, le bannissement ressortissant, au contraire, à l'activité judiciaire de l'umialik ${ }^{2}$.

- On comprend mieux, par là que l'activité judiciaire de l'umialik, chez les Nunamiut, n'est qu'un corollaire de sa prééminence politique : son leadership lui assigne comme devoir essentiel la sauvegarde du groupe ${ }^{3}$, sous tous les aspects. Au niveau de la résolution des conflits, cette mission se formalise en l'accomplis-

Cf. L. POSPISIL, op. cit., 396, 424-427 ; N. ROULAND, op. cit., (Approche du phénomène juridique...), 30-32.

Cf. L. POSPISIL, op. cit., 426.

Cf. supra, p. 33. 
sement d'une fonction maïeutique, que lui permettent et son prestige ${ }^{1}$, et sa connaissance technique du droit coutumier ${ }^{2}$.

- Remarquons, enfin, qu'au point de vue technique, la juridiction exercée par l'umialik sur les matières lui parvenant des niveaux juridiques familiaux ne peut être assimilée à un droit d'appel, car aucune décision n'a pu être arrêtée dans le cas du groupe familial, qui n'a pas commis non plus un déni de justice, puisqu'il est supposé avoir mis en œuvre les moyens dont il disposait. On ne peut non plus parler de droit d'évocation de l'umialik, puisque celui-ci ne se "saisit" pas de l'affaire portée devant lui, comme nous allons le voir. En fait, il [p. 35] s'agit d'une procédure sui generis, irréductible à nos catégories procédurales.

Dans le même sens, ce n'est qu'avec de grandes précautions qu'on doit employer l'expression de "rôle judiciaire de 1'umialik", comme nous l'avons fait plus haut. Il ne s'agit, en effet, que d'une commodité terminologique, dont il nous faut maintenant quelque peu éclairer la signification exacte. En quoi consiste, en effet, l'activité de l'umialik chez les Nunamiut lorsqu'il se voit appelé à intervenir dans la résolution d'un conflit ? ${ }^{3}$

On notera d'abord qu'elle s'inscrit dans un cadre délibérément public, qui manifeste bien que c'est la communauté entière qui est concernée, et qui va décider, l'umialik n'étant que son interprète. En effet, c'est dans le karigi, la maison commune, que va se tenir la délibération ${ }^{4}$. L'umialik joue d'abord un rôle d'enquêteur, se comportant comme une sorte de juge d'instruction : il collecte les informations afin d'établir les faits et d'élucider les motivations des parties. Puis, au bout d'un

1 C'est moins en tant qu'homme de droit que leader politique qu'intervient l'umialik dans les conflits : "... he is first a businessman and, as the effect of this, he acquires a position of influence and respect and he assumes informal leadership in settling legal controversies" (N.J. GUBSER, op. cit., 185).

2 Chez les anciens Germains, le leader intervient dans la résolution des conflits d'une façon très proche de celle de l'umialik (Cf. E.A. THOMPSON, The early Germans (Oxford, 1965), 61), ce qui s'explique par une certaine communauté des traits fondamentaux d'organisation sociopolitique : les leaders, chez les Germains, en temps de paix, ne sont pas des "chefs", mais usent seulement de leur prestige pour régler les conflits et réconcilier les parties ; ce ne sont que des médiateurs, qui ne disposent pas d'un pouvoir de coercition formalisé, et ne sont compétents que pour les conflits internes à leur tribu (ibid., 10). Dans le même sens, on remarquera que l'allotissement périodique des terres entre les différents groupes familiaux (comment ne pas penser à la rotation quinquennale des territoires de chasse chez les Inuit Polaires de Thulé ?) entraînait chez les Germains une continuelle redistribution des moyens de subsistance, empêchant la formation d'inégalités sociales et l'accumulation de richesses entre les mains des leaders. En observateur avisé, César note d'ailleurs que si cette accumulation avait été possible, elle aurait produit des tensions sociales et la concentration du pouvoir dans une autorité centrale (ibid., 8-9).

3 Cf. la description qu'en donne N.J. GUBSER, op. cit., 184-186 ; ainsi que celle de L. POSPISIL, op. cit. (Law and societal structure...), 423.

4 La karigi est l'édifice construit par la bande, qui peut abriter plus de cent personnes. C'est le centre de l'activité de la communauté : on y prend les repas en commun ; les hommes s'y rencontrent, y discutent des chasses collectives aux caribous, y dansent et chantent (Cf. N.J. GUBSER, op. cit., 168). 
certain temps, chacun va exprimer ses idées sur le conflit. Il arrive souvent qu'un ou plusieurs Vieux - qui peuvent avoir été umialik, mais ce n'est nullement obligatoire - appellent le récalcitrant à la raison, le menaçant de se séparer de lui s'il ne se rend pas aux arguments développés par l'umialik. Si, cependant, l'individu demeure récalcitrant, la communauté, dirigée par l'umialik et les Vieux, qui forment une sorte de conseil autour de lui, vont mettre en œuvre des sanctions psychologiques, n'impliquant pas le recours à la force physique : réprimande publique, avertissement, sobriquet qui peut demeurer attaché au coupable toute sa vie. Ce n'est que dans les cas les plus graves (meurtres et récidive) que la sanction sera physique : ostracisme, exécution. Ces quelques éléments appellent plusieurs remarques :

- stricto sensu, l'umialik n'a pas de fonction judiciaire dans le sens que nous donnons à ce mot en droit positif : l'umialik ne juge pas, [p. 36] il n'est qu'un maïeute qui accomplit une mission publique dans un cadre communautaire. En effet, comme nous venons de le voir, ce n'est pas lui qui prend la décision finale, ou du moins il n'est pas seul à le faire ; de même, chaque membre du groupe donne son avis, et les plus âgés peuvent autant que lui enjoindre au coupable de s'amender. D'ailleurs, le fait qu'il importe peu d'avoir ou non été umialik pour le Vieux qui prend la parole est déjà en lui-même significatif. Nous avons ainsi la confirmation du principe énoncé plus haut ${ }^{1}$ suivant lequel le maïeute n'est pas un juge : le seul véritable juge est l'opinion publique, la communauté ${ }^{2}$.

- Cependant, l'umialik n'en participe pas moins activement au processus judiciaire, dans lequel il accomplit un rôle déterminant. Il symbolise la communauté devant laquelle est porté le conflit. Sa connaissance des coutumes lui permet d'éclairer et préciser les options offertes au groupe ${ }^{3}$. Il joue un rôle nettement inquisitoire : il collecte les informations, invite le coupable à s'amender. Il fait partie des personnages qui vont éventuellement décider de la sanction finale. Enfin, son prestige politique et sa position économique lui permettent, plus qu'à tout autre, de faire avancer le débat : pour conquérir sa position d'umialik, il a dû étendre au maximum ses réseaux parentaux, et pour la conserver il a été dans l'obligation de pratiquer un évergétisme à la taille du groupe. Il dispose donc de nombreux appuis, sur lesquels il peut jouer pour déterminer le coupable à avouer et s'amender, notamment en le menaçant de lui retirer sa protection ${ }^{4}$.

- Le moyen de pression principal employé contre le coupable est toujours la référence au groupe : les Vieux et l'umialik le menacent, en lui retirant leur pro-

Cf. supra, p. 25.

Cf. J.W. LAPIERRE, Essai sur le fondement du pouvoir politique (Aix-en-Provence, 1968), 493.

3 Certains indices terminologiques confirment que la notion de leader exprime bien simultanément l'expérience (la connaissance des coutumes) et le prestige politico-économique : les Anciens sont souvent qualifiés des mêmes termes (naskok, anaiyukok) que ceux qui ont acquis une position de leadership. Cf. supra, p. 30, n. 1.

4 Cf. L. POSPISIL, op. cit., 423 : la seule menace de la soustraction de protection par l'umialik suffit, en général, à faire rentrer le coupable dans le droit chemin. 
tection, de le priver en fait de celle de la collectivité. On notera également le caractère très psychologique de ces sanctions.

- Enfin, l'intervention de l'umialik et des Vieux semble fréquente : sur 35 conflits relevés par L. Pospisil, 18 furent réglés par ces derniers ; 2 cas de peu d'importance ne furent pas jugés ; 6 furent réglés par les familles ; les 9 autres cas furent réglés, soit par négociation - 4-, par 1'umialik - 1-, ou par vendetta - 4 1 .

Ces analyses reposent, évidemment, sur un cas spécifique, celui de la société inuit Nunamiut. Il semble, toutefois, qu'il ne s'agisse là que d'un reflet de structures fondamentales des sociétés inuit, qui ne connaissent pas d'autorités judiciaires dans le sens que nous sommes accoutumés à donner à ce mot. (Les Nunamiut font d'ailleurs partie des sociétés alaskiennes qui connaissent, avec celles de Behring, les structures de commandement les plus différenciées et les plus "autoritaires". A fortiori, le raisonnement est-il donc vrai pour des populations aux structures socio-politiques plus lâches et diffuses).

Il paraît, en revanche, plus juste de parler de maïeutes, comme nous l'avons fait : individus qui, par la position particulière qu'ils occupent, aident la communauté à formuler son jugement. Il n'y a d'ailleurs pas à s'étonner de ce caractère maïeutique du rôle judiciaire des leaders : il n'est que le reflet dans une fonction socio-juridique déterminée - la résolution des conflits - des grands principes d'organisation politique des sociétés inuit : le leader n'est pas un chef souverain à la façon de nos monarques européens : il n'est que l'instrument temporaire du groupe, qui le contrôle et peut le déposer, et dont il aide la volonté à se former et s'exprimer. Comme l'écrit fort justement Jean Malaurie "Sociologiquement communautaire, la société arctique est fonctionnellement aristocratique" 2 .

\section{B) TYPES DE PROCÉDURES DE SANCTION DU DROIT SOCIO-PSYCHOLOGIQUES}

\section{Retour à la table des matières}

L'idée d'une sanction "psycho-sociologique" du droit a de quoi éveiller l'étonnement - sinon la suspicion - du juriste occidental, [p. 38] habitué à des procédures judiciaires normalisées et institutionnalisées. Cependant, si l'idée de contrainte demeure fondamentale à l'essence du juridique, cette contrainte peut, dans les sociétés archaïques, se réaliser suivant des modalités plus informelles que dans les sociétés étatiques, mais non moins efficaces, bien que la force physique ne soit pas automatiquement mise en jeu. Avant d'en arriver à ce stade ultime, on préfére-

\footnotetext{
Ibid.

J. MALAURIE, op. cit. (Raids et esclavage), 8.
} 
ra jouer sur l'honneur du coupable ${ }^{1}$, tout en lui laissant la possibilité de s'amender. La finalité profonde de ces démarches est toujours la même : avant d'en arriver à des décisions impliquant un recours à la force brutale, ou à l'élimination, on met en œuvre des moyens qui tendent à rectifier les comportements déviants en entraînant le moins de perturbations possibles à l'équilibre du groupe. L'intérêt privé du fautif et celui de la collectivité se rejoignent donc.

La forme de la sanction importe donc beaucoup moins que son effet: en ce sens nous souscrivons pleinement à l'opinion de L. Pospisil lorsqu'il écrit - contre la théorie classique des juristes "occidentaux" - que la meilleure définition de la norme juridique est fonctionnelle et non pas formelle ${ }^{2}$. Cet auteur exprime, d'ailleurs excellemment, l'idée que la sanction du droit peut être de nature psychologique :

"... I suggest that social control is the important qualification of a legal sanction. Some psychological actions, although of non physical nature, exercise as strong a control as do physical sanctions. Ostracism, ridicule, avoidance, or a denial of favors - sanctions that are sometimes very subtle and informal - nevertheless may become more drastic that the corporal punishment to which we tend to attach over importance even in our own culture" ${ }^{3}$.

[p. 39]

Applicables à une grande partie des sociétés archaïques ${ }^{4}$, ces principes le sont sans nul doute aux sociétés inuit, sociétés de refoulement où, pour l'individu, tout

1 HOLM (cité par C.C. HUGHES, op. cit., 366) rapporte qu'à Ammassalik (Côte Est du Groënland) est en vigueur un code non écrit accepté tacitement par tous et sanctionné par le déshonneur.

2 Cf. L. POSPISIL, op. cit., (The Nature of Law), 751.

3 Ibid., 751. Cf. aussi : T. PARSONS, "The Law and social control", dans Law and Sociology. Exploratory essays (edit. W.M. Evan, New York, 1962), 59-60 ; "A legal order shall rather be said to exist wherever coercive means, of a physical or psychological kind, are available ; i.e. wherever they are at the disposal of one or more persons who hold themselves ready to use them for this purpose in the case of certain events ; in other words, wherever we find a consociation specifically dedicated to the purpose of "legal coercion" (Max WEBER, Law in economy and society (Cambridge, 1954), 17, cité par W.M. EVAN, "Public and private legal systems", dans Law and Sociology (op. cit., 166).

4 "La plupart des sociétés sans écriture sont caractérisées par l'absence d'une force organisée pour infliger les peines, et par le recours à une application fréquente de la sanction diffuse que constituent la réprobation du groupe et son acquiescement à la vengeance privée" (A. DORSINFANG-SMETS, "Réflexions sur les modes de preuve dans l'action judiciaire des sociétés dites primitives", dans Recueils de la Sté Jean Bodin : La preuve, XVII - 3 (Bruxelles, 1963, 20). 
est contrainte psychologique, théâtralité ${ }^{1}$. Les ethnographes de l'Arctique savent combien le sourire quasi-perpétuel de l'Inuk est l'effet d'un processus de sublimation $^{2}$. Il est donc normal qu'au niveau de la résolution des conflits, on assiste à la mise en œuvre de ces mécanismes socio-psychologiques. On se souvient d'ailleurs de la nature très psychologique des sanctions chez les Nunamiut ${ }^{3}$. On comprend d'ailleurs fort bien que la menace de la sanction soit déjà en elle-même une sanction. E.W. Nelson rapporte ainsi que, dans la région de Behring, celui qui a commis un meurtre est dans une situation intenable, car redoutant la revanche, il est sans cesse aux aguets. Nelson ajoute même que l'individu est reconnaissable à la seule expression de son regard ... ${ }^{4}$ La nature abstraite de la sanction est d'ailleurs d'autant plus prononcée que, comme nous le [p. 40] signalions plus haut ${ }^{5}$, il y a liaison entre l'ordre social et surnaturel.

Quels sont les principaux types de sanctions socio-psychologiques ? ${ }^{6}$ On peut en distinguer plusieurs, dans l'ordre d'une contrainte de plus en plus élevée, qu'on retrouve dans de nombreuses sociétés inuit, dispersées sur toute l'étendue de l'Arctique.

- Une première série repose sur la dérision et la réprimande :

- chez les Inuit du Caribou, les fautes les moins graves sont traitées par la dérision. L'individu paresseux qui chasse insuffisamment pour sa famille, laquelle doit être alors prise en charge par le groupe, devient l'objet d'un chant satirique

1 L'aspect ritualiste des procédures judiciaires a pour but de donner à la solution dégagée une autorité qui frappe l'opinion publique : même dans nos sociétés modernes, on peut sans peine constater cet effet (Cf. A. DORSINFANG-SMETS, op. cit., 35).

2 Cf. l'ouvrage de J.L. BRIGGS, si bien nommé Never in anger. Portrait of an Eskimo family (London, 1971).

Cf. supra, p. 36.

4 Cf. E.W. NELSON, The Eskimo about Behring Strait (Washington, 1899), 293. Dans une optique similaire, on peut penser à la coutume du "baiser de mort", en vigueur chez les membres de la Mafia : celui qui a trahi la Mafia vit dans la crainte d'une vengeance qu'il sait inéluctable, mais qui peut être différée de plusieurs années. Ce retard constitue déjà en lui-même une partie de la sanction. Au point que lorsque l'individu reçoit d'un de ses anciens congénères le "baiser de mort" - qui signifie que la vengeance est imminente - il en est souvent soulagé.

5 Cf. supra., pp. 26-27. Les Inuit Polaires pensent ainsi que la maladie est toujours la conséquence de la violation d'un tabou par le malade (Cf. E. HOLTVED, op. cit., 151). V. STEFANSSON (op. cit., 272-273) rapporte qu'un jour un homme, pour se soustraire aux règles de partage collectif du gibier - qui touchent plus particulièrement les animaux d'une taille relativement importante - n'avertit pas ses camarades de chasse au moment où il tua la bête - ainsi que l'exigeait la coutume - et leur dit par la suite qu'il avait seulement tué un petit phoque. Mais il fut découvert, sa viande lui fut confisquée après une séance publique de reproches, et il devint aveugle. D'ailleurs, dans les sociétés archaïques, la maladie ou la mort du coupable peuvent résulter d'une autopunition de ce dernier, qui peut s'estimer condamné par les forces surnaturelles (Cf. G. SAWER, Law in Society, Oxford, 1965, 39).

On voit, par ces quelques exemples, combien la sanction, tout en étant psychologique et abstraite, peut être sociologiquement efficace.

6 N.H.H. GRABURN, op. cit., 49-56, en cite plusieurs. 
lors des séances de danses, et encourt de nombreuses plaisanteries. Ces actions ne cesseront qu'à partir du moment où il modifiera son comportement ${ }^{1}$.

- Chez les Inuit de Kangirsujjuaq (Nouveau-Québec), le recours à la dérision précède également la mise en œuvre de sanctions plus dures (ostracisme, privation de parts de gibier, liquidation physique) ${ }^{2}$.

[p. 41]

- Les Inuit du district du Keewatin (T.N.O.) usent aussi très fréquemment de la moquerie : dans leurs rapports quotidiens, dans des chants improvisés lors des réunions dans la grande maison d'hiver, dans des compétitions de chants formelles dans le même cadre ${ }^{3}$. Les Netsilik recourent d'ailleurs plus volontiers encore que les Caribou à la moquerie, ces derniers préférant la fuite devant le perturbateur, auquel ils appliquent ainsi une forme d'ostracisme ${ }^{4}$.

- On a vu que chez les Nunamiut la peur d'être coupé de la protection des leaders du groupe suffisait souvent à amender le coupable ${ }^{5}$.

- Chez les Inuit de la région de Behring, le voleur subit un blâme public dans la maison commune, le kashim $^{6}$. La même règle s'observe chez les Inuit Chugach ${ }^{7}$.

- Une seconde modalité consiste dans l'adresse au coupable en termes d'injure.

- Une forme subtile d'injure - qu'on peut également assimiler à une sorte d'ostracisme - consiste à ne plus s'adresser à l'individu par son terme de parenté (Nord-Ouest de la baie d'Hudson) ${ }^{8}$.

- À Ammassalik, K. Rasmussen rapporte que celui qui tenterait de se soustraire aux règles de partage collectif du gibier serait appelé "le cupide", ce qui est une injure que personne ne saurait supporter ${ }^{9}$.

1 Cf. F. MOWAT, Mours et Coutumes des Esquimaux Caribous (Paris, 1953), 181, et J. GABUS, op. cit., 140.

Cf. B. SALADIN D'ANGLURE, op. cit., 187-188.

Cf. M. GLUCKMANN, op. cit., 303.

Cf. G. VAN DEN STEENHOVEN, "The Eskimos", dans Recueils de la Sté Jean Bodin : Gouvernés et gouvernants, XXII-I (Bruxelles, 1969), 348-351.

Cf. supra, p. 36.

Cf. E.W. NELSON, op. cit., 293.

Cf. K. BIRKET-SMITH, op. cit. (The Chugach Eskimo), 98. (Sur les séances de réprimande en général, cf. N.H.H. GRABURN, op. cit., 53).

Cf. J.L. BRIGGS, op. cit., 215.

9 Cf. "Knud Rasmussen's Posthumous notes on the life and doings of East Greenlanders in olden times", M.o.G., 109-1 (Copenhagen, 1938), 48. 
- On a vu ${ }^{1}$ que chez les Nunamiut, le coupable peut aussi se voir affublé d'un sobriquet durant toute sa vie. Chez ces mêmes Nunamiut, le voleur peut être désigné par le nom de l'objet qu'il a volé ${ }^{2}$.

[p. 42]

- Chez les Inuit Polaires de Thulé, par la voie des cancans, le coupable se voit affublé de qualificatifs très cruels) ${ }^{3}$.

Chez certaines populations Inuit, l'amendement du coupable peut se concrétiser sous une forme particulière, qui revêt elle aussi un aspect très nettement sociopsychologique, et même psycho-thérapeutique ${ }^{4}$ : la confession publique. Elle est attestée chez les Inuit centraux ${ }^{5}$ (notamment Caribou) ${ }^{6}$, ceux de la côte Ouest du Groënland ${ }^{7}$, mais est absente sur la côte Est du Groënland ${ }^{8}$. K. Rasmussen en donne une description détaillée chez les Inuit Iglulik : ${ }^{9}$ pressée par le shaman et l'assistance, la femme avoue petit à petit les fautes qu'elle a commises. Elle commence par une déclaration d'ordre général, disant que ses pensées et ses actions ont été mauvaises. Puis le shaman lui fait avouer, les unes après les autres, les violations de tabous qu'elle a commises : elle a fumé une pipe qu'elle ne devait pas fumer, mangé du caribou dans une période tabou, travaillé une tête de harpon en touchant certaines parties de l'animal à un moment où cela était interdit, peigné ses cheveux après avoir accouché - ce qui est tabou -, etc. Parfois, c'est l'assistance elle-même, sans attendre l'aveu de la coupable, qui indique au shaman le tabou qu'elle a enfreint.

Chez les Inuit Polaires de Thulé "...si le causeur de tort ne s'humilie pas en reconnaissant ses faiblesses, son impuissance - et il lui suffira de le faire devant quelques témoins - force lui sera de s'isoler et de vivre à part". ${ }^{10}$

Tous ces types de sanction psychologiques paraissent universellement répandus au sein des sociétés Inuit, puisqu'on les trouve aussi [p. 43] bien chez les riches sociétés behringiennes que dans les communautés archaïques (Caribou et Iglulik). Cependant, toutes ces formes ne se trouvent pas au complet partout : on a vu ainsi que les Inuit de Est du Groënland ne connaissent pas la confession publique.

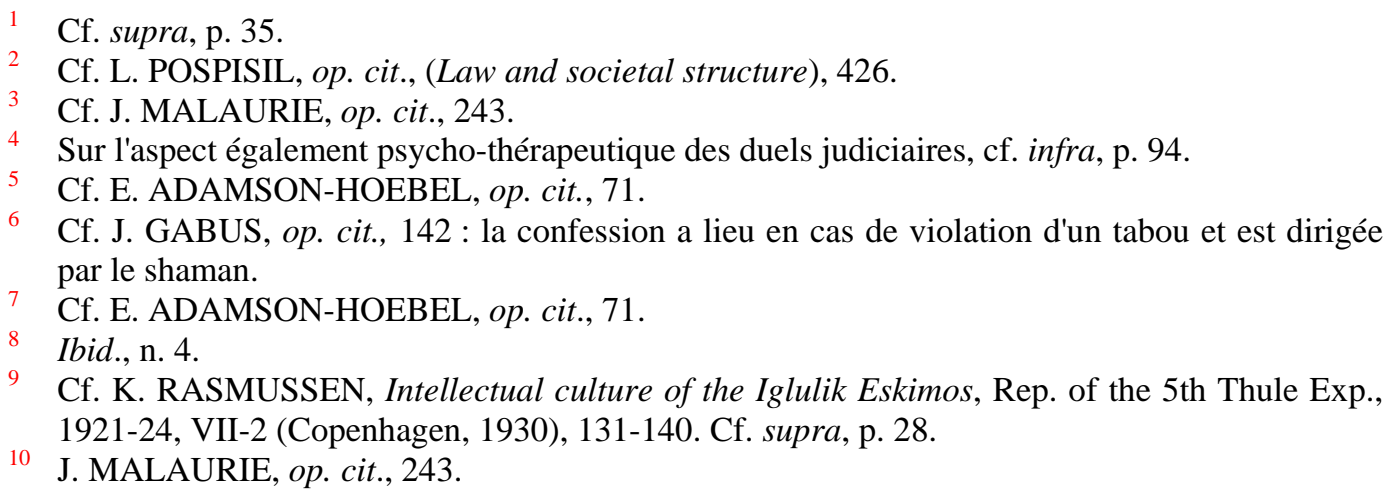


Pour les cas plus graves, où ceux dans lesquels les procédés décrits ci-dessus n'ont pu apporter une solution au conflit, on en vient à l'ostracisme, appliqué selon des variantes plus ou moins "dures". L'ostracisme, au moins dans sa forme la plus brutale - l'abandon ou le bannissement - n'est certes pas qu'une sanction psychologique : dans l'Arctique, l'individu abandonné par le groupe a peu de chances de survivre, et c'est donc dans sa chair que cette mesure l'atteindra.

Néanmoins, l'ostracisme, surtout dans ses formes "douces" a incontestablement une portée psychologique : privé de la protection du groupe, l'individu se retrouve désorienté ${ }^{1}$, cela d'autant plus que toute la vie sociale inuit, comme nous l'avons déjà dit, est axée sur la soumission de l'individu au groupe.

L'ostracisme comporte donc toute une gamme de procédés :

- Nous avons vu que le fait de ne plus s'adresser à un individu par son terme de parenté (Nord-Ouest de la baie d'Hudson) peut être considéré comme une forme subtile d'ostracisme ${ }^{2}$.

- L'ostracisme peut consister dans une mise à l'écart temporaire : l'individu est mis au ban du groupe, mais il en fait encore partie. C'est une sorte de sursis avec mise à l'épreuve. Ainsi, chez les Inuit Netsilik, si deux individus en conflit n'arrivent pas à trouver une [p. 44] solution de compromis, l'un d'entre eux peut avoir à aller s'installer à l'autre extrémité du camp ${ }^{3}$. Chez les Nunamiut (Nord-Alaska), la communauté réunie dans le karigi peut décider de ne plus parler pendant un certain temps à celui qui a un comportement asocial ${ }^{4}$. Chez les Inuit Polaires de Thulé, celui qui refuse la confession de ses torts devra s'isoler et vivre à part ${ }^{5}$.

- Si la mise à l'écart temporaire ne réforme pas le coupable, ou dans le cas d'infractions graves ${ }^{6}$, l'ostracisme revêt alors une forme "dure" et devient un véritable bannissement :

Dans nos sociétés modernes, l'emprisonnement du délinquant revêt incontestablement cet aspect. Il constitue d'abord une mesure de défense sociale, c'est-à-dire qu'il met la société à l'abri des actes délictueux que pourrait commettre de nouveau le coupable. Mais l'incarcération est aussi un châtiment : non seulement en ce qu'elle prive l'individu qui en est l'objet de sa liberté d'action, mais parce qu'elle le coupe du milieu social dans lequel il évoluait avant son emprisonnement. C'est la raison pour laquelle certaines réformes pénitentiaires échouent : on sait bien que dans les établissements carcéraux "modèles", où le prisonnier dispose d'un confort réel mais vit dans un isolement très prononcé, le taux de suicides est beaucoup plus élevé que dans les anciennes prisons, insalubres, mais où peut se reconstituer, dans le milieu carcéral, une hiérarchie "parallèle", fondée sur le prestige, l'expérience, etc ...

Cf. supra, p. 41.

Cf. A. BALIKCI, The Netsilik Eskimo (New York, 1970), 192.

Cf. W.H. OSWALT, op. cit., 178.

Cf. J. MALAURIE, op. cit., (Les derniers rois de Thule), 243 ; et supra, p. 43.

"The violation of a tabu is a sin supernaturally sanctioned ; willful and repeated sinning becomes a crime legally punished. Penalty : exile, in the Arctic" (E. ADAMSON-HOEBEL, op. cit., 74). 
- Chez les Inuit de Sugluk (Nord de la péninsule d'Ungava), les paresseux, ou ceux qui séduisent les femmes mariées sont incités par la communauté à s'en aller. S'ils ne le font pas, les leaders du groupe décident leur exécution ${ }^{1}$.

- Chez les Nunamiut (Nord-Alaska), le groupe peut décider l'ostracisme 2 .

- Les Inuit du Caribou pratiquent une forme originale d'ostracisme : au lieu de bannir le coupable en l'expulsant du groupe, c'est le groupe lui-même qui l'abandonne ${ }^{3}$, en levant le camp par surprise, durant la nuit, sans l'emmener, ou en l'excluant d'une expédition.

On peut enfin se demander, au terme de l'exposé de ces divers moyens de sanction socio-psychologiques, comment le groupe, avant de décider de leur mise en œuvre, a pu être informé de l'identité du coupable et de la nature de ses infractions. Dans ces communautés de taille restreinte, l'information circule vite :

"(Dans ces sociétés) la liberté n'existe pas (...) il y a des espions partout : (...) les petits enfants, qui transmettent ce qu'ils [p. 45] voient d'une maison à l'autre" ${ }^{4}$. Le phénomène des "cancans", dans son aspect psycho-sociologique et son rôle dans la résolution des conflits, a d'ailleurs bien été noté par J. Malaurie à propos des Inuit Polaires de Thulé :

"Si le dire est informatif et répressif, s'il sanctionne les conflits, le propos est le plus souvent de caractère exploratoire : on lance une information pour en savoir davantage, mais aussi par désir de se distraire. Dans la société d'ennui qu'est la société inuit, le cancan est une pulsion susceptible de créer l'événement désiré : on est à l'affût d'un bruit, d'une rumeur qui, pour un temps, changera le goût de la vie ${ }^{5}$ (...) Dans cette société qui s'oblige à la cohésion, la parole est un instrument de contrainte obligeant l'homme à n'agir que dans le sens de la tradition et à se dépasser. La société de groupe est répressive ; la parole - je l'ai déjà dit - expression du groupe-arbitre (on ne dit jamais : je pense, mais les Inuit pensent, le je étant proscrit dans la pensée même) est une arme puissante. L'Inuit doit toujours dire non pas ce qu'"il" ressent personnellement, mais ce que ressent le groupe tout entier. La pensée inuit est ainsi comme le plus petit dénominateur commun à ces diverses fractions familiales, parfois individuelles. La parole par les cancans, les propos, corrige et redresse. Les mous, les faibles, les parasites, les fourbes sont critiqués comme dans une société communiste; les mauvais travailleurs sont marqués du doigt et mis au pilori : "Ils nous apportent le malheur, ils ne respectent pas les règles, méprisent les tabous..." "Par des qualificatifs d'une extraordi-

\footnotetext{
Cf. N.H.H. GRABURN, Eskimos without igloos (Boston, 1969), 61.

Cf. W.H. OSWALT, op. cit., 178.

Cf. G. VAN DEN STEENHOVEN, op. cit.

J. MALAURIE, émission Radioscopie.

J. MALAURIE, op. cit., (Les derniers rois de Thulé), 237.
} 
naire cruauté, les carences de ces atypiques sont dénoncées dans tout le territoire" ${ }^{1}$.

[p. 46]

Cette analyse a été faite pour les Inuit Polaires de Thulé. Mais elle est valable aussi pour les Netsilik : chez ces derniers, afin de prévenir les conflits, chaque individu dont le comportement paraît atypique est entouré d'un cercle qui le surveille, et commente ses agissements ${ }^{2}$.

Le cancan, le commérage, sont donc élevés dans les sociétés inuit à la hauteur d'une institution : ce sont eux qui diffusent les informations, exercent une pression sur l'atypique, et préparent la communauté à pouvoir au mieux remplir ultérieurement son rôle de juge. Dans ces sociétés restreintes démographiquement, et où l'individu est constamment sous la surveillance du groupe ${ }^{3}$, ces commérages épargnent la nécessité de règles ayant pour but d'assurer la publicité des actes juridiques, dont on connaît l'importance chez nous.

On ne saurait manquer de souligner ici combien, sur ce point précis, il y a de ressemblance entre ces sociétés inuit et les communautés villageoises de notre Moyen-Âge français : dans un livre récent E. Leroy-Ladurie, se penchant sur la vie d'un village de montagne ariègeois aux $\mathrm{XIII}^{\mathrm{e}}$ et $\mathrm{XIV}^{\mathrm{e}}$ siècles, a montré là aussi l'importance des commérages féminins dans la vie sociale : "...elles (les servantes) sont l'un des piliers du système d'informations et l'un des canaux de la transmission culturelle descendante, dans le village (...) Borborygme géant, la rumeur féminine monte de chaque village (...) Dense et diurne, la sociabilité féminine est en outre répétitive, voire quotidienne ... les paysannes occitanes entre elles éprouvent un irrépressible besoin de saisir et garder la parole (...) La sociabilité féminine comme telle ne vise pourtant que de façon marginale à récupérer des pouvoirs et des biens... Elle se situe au niveau de l'information et de la communication, plus qu'à celui de la puissance proprement dite (...). Espionnage actif, donc. Mais aussi manie du questionnaire. Curieuses, les femmes de Montaillou, surtout les jeunes, procèdent, entre elles, [p. 47] par feu roulant de questions" ${ }^{4}$. (Un des moments où

1 Ibid., 242-243. N.H.H. GRABURN, op. cit. (Eskimo law) 53, fait observer que les commérages ne sont pas réellement une réaction à un conflit, car ils touchent constamment des individus coupables, mais aussi innocents. Il est vrai que les commérages ne sont pas en eux-mêmes une procédure judiciaire : mais ils constituent un des moyens par lesquels la communauté peut connaître, puis juger des conflits quand ceux-ci se révèlent réels, et ne procèdent pas de l'imagination : le commérage n'est pas a priori la médisance. D'autre part, il serait illusoire de croire que les procédures judiciaires ne touchent que les coupables...

Cf. A. BALIKCI, op. cit.., 185.

3 L'habitat et les usages facilitent cette surveillance : un iglou n'est jamais fermé, et le visiteur doit toujours être bien accueilli. À côté de la promiscuité sexuelle chez les Inuit - dont une certaine littérature a trop parlé - il faut souligner l'importance de cette promiscuité psychologique qui est un des ressorts de la vie sociale.

4 E. LEROY-LADURIE, Montaillou, village occitan de 1294 à 1324 (Paris, 1975), 379, 382, 383, 384, 386. 
s'épanouit tout particulièrement cette sociabilité féminine est celui des séances d'épouillage collectif) ${ }^{1}$.

(Durant le Haut Moyen-Âge, on notera que la justice étatique fait une large place aux informations et au jugement des gens du $\mathrm{cru}^{2}$ : on estime, en effet, qu'en raison de leurs conditions de vie, ils étaient les mieux à même de connaître exactement les faits reprochés et de porter une appréciation sur les responsabilités en cause).

On serait tenté de déduire de tout ce qui précède que les modes de sanction juridique inuit, par leur nature psychologique, sont comme insaisissables par la mentalité juridique occidentale. On doit faire observer que ces sanctions sociopsychologiques, pour importantes qu'elles soient, ne voient pas moins fonctionner à côté - ou en dessus - d'elles des modes institutionnels de sanctions plus formalisés, et de ce fait plus accessibles au juriste occidental. Distincts des précédents, ils n'en présentent pas moins une caractéristique commune : la préservation des intérêts du groupe par l'intervention de ce dernier. Ce sont ces problèmes qu'il nous faut maintenant étudier dans le second chapitre de cette étude.

Ibid., 382.

Citons-en deux exemples : - la juridiction des rachimbourgs, à l'époque mérovingienne : dans le cadre du mallus (le tribunal comtal de droit commun), la justice a un caractère très populaire. En effet, si ce tribunal est présidé par le comte ou son remplaçant, il est pour le reste composé d'hommes libres choisis sur place par le comte lors de chaque session : les rachimbourgs. - "enquête de pays" (époque carolingienne) : en cas de crime grave commis par des individus que personne n'osait dénoncer, le roi ordonnait au comte de réunir un jury d'hommes de bien, résidant sur les lieux des faits délictueux, et de les sommer de dénoncer les coupables. (Sur ces questions, cf. N. ROULAND, Cours d'Histoire du Droit Français (Aix-en-Provence, 1976), $51,57)$. 
[p. 49]

\section{CHAPITRE DEUX}

\section{ACTION DU GROUPE ET INTÉRÊT PUBLIC DANS LES PROCESSUS JURIDIQUES INSTITUTIONNELS DE CONTRAINTE}

\section{$\underline{\text { Retour à la table des matières }}$}

Dans les sociétés archaïques élémentaires, il serait illusoire de déduire de la primauté du groupe qu'il intervient sans cesse directement dans la résolution des conflits qui peuvent s'élever entre ses membres. Le règlement de certains conflits est laissé à l'initiative familiale, qui s'exerce dans le cadre de la vendetta. Cependant, le groupe s'efforce de limiter les conséquences néfastes de l'enchaînement de vengeances qui peut en résulter : il intervient alors indirectement.

Dans d'autres cas, le groupe intervient directement en décidant la liquidation du perturbateur, ou en institutionnalisant des modes de règlement pacifique par le biais des duels judiciaires. Dans ces deux derniers cas, on peut parler d'intervention directe du groupe, ou d'action publique.

Nos propos s'ordonneront donc autour de cette subdivision :

Section I : Mode privé de solution des conflits : la vengeance familiale et sa limitation par le groupe

Section II: Modalités de l'action publique : intervention directe du groupe dans le règlement des conflits 
[p. 50]

\section{Section I : $\quad$ MODE PRIVÉ DE SOLUTION DES CONFLITS : LA VENGEANCE FAMILIALE ET SA LIMITATION PAR LE GROUPE ${ }^{1}$}

\section{$\underline{\text { Retour à la table des matières }}$}

La vengeance par le sang était une obligation fondamentale pour la parenté la plus proche. À cette loi, nul ne pouvait se soustraire" 2. Cette affirmation, énoncée par l'auteur quant aux populations behringiennes, est valable pour l'ensemble des sociétés inuit ${ }^{3}$. Il est d'ailleurs quasi général que, dans les sociétés a-étatiques, ou bien dans celles où les institutions étatiques sont trop faiblement développées, ou encore dans celles où les structures politiques ne se sont pas dégagées des hiérarchies parentales, certaines fautes soient soumises à la loi de la vengeance privée, exercée dans un cadre familial. On en trouve sans peine des exemples dans les droits antiques et médiévaux :

- À Athènes, la vengeance du meurtre peut s'exercer sur tous les membres du groupe familial du meurtrier. L'interdiction lancée contre le meurtrier dans l'Agora peut être prononcée par tous les parents de la victime jusqu'au degré de cousin germain exclusivement, les autres pouvant cependant concourir à la poursuite. Ce privilège est aussi un devoir, car il a un caractère obligatoire ${ }^{4}$.

- Dans la Rome archaïque, les gentes règlent elles-mêmes leurs conflits : seuls les crimes qui étaient aussi des fautes religieuses relevaient du roi, chef de la communauté ${ }^{5}$.

- Le Moyen-Âge féodal a vécu, comme on le sait, sous le signe de la vengeance privée ${ }^{6}$ et ce n'est qu'au $X V^{\mathrm{e}}$ siècle que le roi a pu établir [p. 51] en France sa paix générale, et abolir ainsi le régime de la guerre privée.

Pour une étude générale de la vengeance sanglante chez les Inuit, cf. H. KONIG, op. cit., (Der Rechtsbruch...), 771-772. Sur les caractères juridiques généraux de la vengeance privée, cf. G. STEFANI - G. LEVASSEUR, Droit Pénal Général (Paris, 1966), 55.

J. MALAURIE, op. cit. (Raids et esclavage), 5.

Dans le même sens, cf. H. KUNIG, op. cit., 508.

4 Cf. L. BEAUCHET, Histoire du droit privé de la République athénienne, I (Amsterdam, 1969), 14-16.

Cf. J. GAUDEMET, op. cit., 271.

6 Cf. M. BLOCH, La société féodale (Paris, 1968), 185-186 ; "...dès que surgit un péril, malheur à l'homme seul". (R. FOSSIER, Histoire sociale de l'Occident médiéval (Paris, 1970), 123). Pour une optique comparatiste, cf. N. ROULAND, op. cit., 58-60. 
La vengeance privée familiale semble donc bien constituer un mode de sanction fondamental des sociétés à dominante archaïque ${ }^{1}$. Nous en examinerons successivement les causes et les modalités.

\section{A) LES CAUSES}

Retour à la table des matières

Chez les Inuit, dans l'immense majorité des cas, la cause de la vengeance sanglante réside dans des conflits tournant autour de l'appropriation de la femme, de relations maritales ou adultérines ${ }^{2}$. La vengeance suit d'autant plus fréquemment que ces conflits entraînent souvent un meurtre ${ }^{3}$. Les occasions de déclenchement de cette vengeance peuvent, en effet, se ramener à une série de cas :

- Rapt de la femme ${ }^{4}$ : un cas Iglulik montre que le mari, non content de tuer celui qui avait commis le rapt, essaya aussi de tuer ses deux frères ${ }^{5}$, ce qui semble bien confirmer que c'est autant le crime qui doit être vengé que le criminel puni. Ce principe est le ressort de la vengeance familiale, qui repose sur une responsabilité objective du groupe familial, plus que sur celle du criminel. En effet, bien que les premières manœuvres de vengeance se portent de préférence vers le coupable, si ce dernier est hors d'atteinte, les membres de sa famille devront payer pour lui, car les Inuit considèrent que l'âme de la victime n'est pas en repos tant que le sang du criminel ou d'un de ses proches n'a pas coulé ${ }^{6}$. Il y a donc responsabilité objective et collective. On [p. 52] peut citer d'autres cas de rapt. Chez les Inuit centraux ${ }^{7}$ : chez les Netsilik, qui sont d'autant plus fréquemment obligés de recourir au mariage par rapt que le taux élevé d'infanticides des filles entraîne chez eux une rareté des femmes ${ }^{8}$. G. Van den Steenhoven confirme cette donnée

1 Qui peut évidemment subsister à titre résiduel dans des sous-groupes localisés d'une société globale étatique : il en est, par exemple, ainsi de la vendetta corse.

2 H. KONIG, (op. cit., 509) voit plus dans le meurtre que dans les femmes la cause de la vengeance sanglante.

Cf. K. RASMUSSEN, Du Groënland au Pacifique, 285.

Cf. E. ADAMSON-HOEBEL, op. cit., 85.

Cf. H. KÖNIG, op. cit. (Das Recht der Polarvölker), 115.

6 Cf. H. KÖNIG, op. cit., 512. Chez les Inuit d'Ammassalik tout au moins, la vengeance, en effet, n'est pas seulement une question d'honneur familial, mais comporte une dimension religieuse : si les parents du mort ne s'y livraient pas, le mort lui-même s'en chargerait, et les conséquences en seraient encore plus terribles. D'ailleurs, le meurtrier, après avoir commis le crime, découpait le cadavre de sa victime et en dispersait les membres afin d'éviter son retour, et sa vengeance (Cf. K. RASMUSSEN, op. cit., (Life and doings of East Greenlanders...), 9091).

7 Cf. E. ADAMSON-HOEBEL, op. cit., 88.

8 Cf. L. GUEMPLE, "Eskimo band organization and the "DP Camp" hypothesis", dans Arctic Anthropology, IX-2 (1972), 101. 
en notant que le meurtre a souvent pour raison l'accaparement d'une femme ${ }^{1}$. On observe aussi des cas analogues à Ammassalik (Côte Est Groënland) ${ }^{2}$.

- Relations maritales : R. Gessain note qu'à Ammassalik (Côte Est Groënland), la brutalité du mari envers la femme peut être source de vendettas ${ }^{3}$.

- Refus de la famille de la femme désirée de consentir au mariage : un prétendant ainsi éconduit, Igjugarjuk, leader chez les Padlermiut (Inuit Caribou), tua toute la famille pour avoir celle qu'il convoitait ${ }^{4}$.

- Adultère ${ }^{5}$ : les Asiagmiut (Inuit du Cuivre) doivent décider l'extermination complète d'une famille (1905) pour éviter l'enchaînement des vendettas ; le fait générateur résidait dans le refus du mari de consentir à ce qu'un tiers ait des relations sexuelles régulières avec sa femme ${ }^{6}$. K. Rasmussen rapporte un conte des Inuit Caribou dans lequel un homme qui avait voulu coucher avec la femme de son frère est tué par ce dernier ; mais le meurtrier est ensuite tué par le frère de sa victime ${ }^{7}$. [p. 53] Ce cas est intéressant, car il montre bien qu'ici c'est l'appartenance familiale qui joue de façon quasi automatique, et que l'acte criminel est apprécié de façon objective : au moins dans ce cas, le seul accomplissement du crime a suffi, que les motifs en aient ou non paru justiciables. Cette constatation ne semble cependant pas être de portée générale : W.A. Oswalt note, à propos des Inuit Tareumiut (Alaska) que, chez eux aussi, les femmes sont les causes les plus fréquentes des meurtres, mais que la famille de la victime, sauf si elle veut en faire une opération de prestige en montrant sa force, ne déclenche pas de représailles si elle estime que le meurtre est justifié ${ }^{8}$ : la responsabilité est donc là appréciée de façon subjective. L'observation d'Oswalt est confirmée - à propos du rapt - de façon plus générale pour tous les Inuit du Nord de l'Alaska par R.F. Spencer : le rapt de la femme n'est pas une cause de vengeance sanglante, encore que le mari lésé, pour récupérer sa femme, puisse faire appel à l'aide de ses parents ${ }^{9}$. Comment expliquer cette différence dans les observations ? La réponse est difficile à apporter, mais peut-être peut-on proposer une hypothèse. D'une façon générale, on constate dans l'histoire juridique que moins une population est archaïque, moins la responsabilité pénale est appréciée de façon objective : l'évolution va dans le sens de la subjectivité du délit, de la considération de l'intention à côté de l'élément matériel. On sait, d'autre part, que les Inuit Caribou sont parmi

1 Cf. G. VAN DEN STEENHOVEN, op. cit., (Legal concepts) 48. Cf. dans le même sens, D. PRYDE, op. cit., 60-62.

Cf. R. GESSAIN, Ammassalik ou la civilisation obligatoire (Paris, 1969), 56.

Cf. R. GESSAIN, op. cit., 41. Dans le même sens, cf. un cas pratique rapporté par P.E. VICTOR, Boréal (Paris, 1972), 196-197.

Cf. E. ADAMSON-HOEBEL, op. cit., 85.

Cf. R. KJELLSTRÖM, Eskimo Marriage (Lund, 1973), 176.

Cf. E. ADAMSON-HOEBEL, op. cit., 86.

Cf. K. RASMUSSEN, observations on the intellectual culture of the Caribou Eskimos, Rep. of the 5th Thule Exp. 1921-24, VII-2 (Copenhagen, 1930), 107.

Cf. W.H. OSWALT, op. cit., 182.

Cf. R.F. SPENCER, The North Alaskan Eskimo (Washington, 1959), 80. 
les plus archaïques des populations inuit, de taille très restreinte, et vivant dans des conditions économiques très précaires. Au contraire, les Tareumiut appartiennent aux Inuit alaskiens côtiers, qui vivent de la riche pêche à la baleine : leurs structures socio-politiques sont beaucoup plus formalisées. Parvenus à un stade culturel plus développé que les Caribou, du fait du contexte économique, il n'est pas impossible que leur appréciation des délits s'en soit ressentie. De plus, leur relative aisance démographique peut aussi expliquer que les affaires impliquant des femmes soient considérées comme moins graves que dans des sociétés plus réduites, et donc ne nécessitant pas la mise en œuvre des vendettas familiales.

[p. 54]

Cependant, les affaires de femme ne sont pas l'unique cause de la vengeance sanglante. Le meurtre, en dehors de tout rapport direct avec l'appropriation d'une femme, en est le second motif ${ }^{1}$ :

- Chez les Inuit Nunivak (Nord-Ouest Alaska), le meurtre n'est jamais sanctionné par la communauté, mais par le groupe familial de la victime. Le vengeur est le fils aîné qui, s'il est mineur au moment du crime, est élevé dans l'idée qu'il devra un jour venger son père ${ }^{2}$. (Cette règle de la sanction différée en cas de minorité du vengeur peut d'ailleurs s'appliquer à celui qui est l'objet de la vengeance : à propos des Inuit Caribou, K. Rasmussen rapporte un conte dans lequel les vengeurs attendent que le descendant du criminel soit parvenu à l'âge adulte pour se décider à exercer sur lui la vengeance, demandant même à son père adoptif s'il estime le moment venu ${ }^{3}$ ).

- Chez les Inuit Netsilik, K. Rasmussen rapporte un cas dans lequel les parents d'un homme tué par son compagnon de chasse ne furent retenus d'exercer la vengeance que par crainte de la justice des Blancs ${ }^{4}$.

- Chez les Nunamiut (Alaska), c'est aussi à la famille de venger le meurtre ${ }^{5}$.

- À Ammassalik également, en cas de meurtre d'un parent linéaire ${ }^{6}$.

Cependant, le meurtre, contrairement à certaines affirmations ${ }^{7}$, n'entraîne pas toujours la vendetta : il en est ainsi chez les Inuit du Cuivre, Iglulik, et ceux de

1 K. BIRKET-SMITH, op. cit., (Mœurs et coutumes) note que, d'une façon générale, le crime ne peut être réglé que par la vendetta.

2 Cf. W.H. OSWALT, op. cit., 183. Dans le même sens, cf. K. BIRKET-SMITH, op. cit., (Mours et coutumes), 178 ; E. ADAMSON-HOEBEL, op. cit., 87.

Cf. K. RASMUSSEN, op. cit., (Intellectuel culture of the Caribou Eskimos), 106-107.

4 Cf. K. RASMUSSEN, The Netsilik Eskimos. Social life and spiritual culture, Rep. of the 5th Thule Exp. 1921-24, VIII 1-2 (Copenhagen, 1931), 20-21.

Cf. N.J. GUBSER, op. cit., 151.

Cf. C.C. HUGHES, op. cit., 373.

Cf. supra, p. 51, n. 2. 
l'Est du Groënland. Chez ces dernières populations, la vendetta est facultative et dépend surtout de la force [p. 55] des parties en présence ${ }^{1}$. On peut penser que, là encore, la cause de ces exceptions réside dans l'archaïsme et la précarité économique et démographique des populations considérées : dangereuses pour l'équilibre du groupe par leurs effets dévastateurs, ces vendettas peuvent, en effet, être très mal tolérées dans les populations déjà très menacées par le seul milieu naturel. Peut-être ce raisonnement trouve-t-il une preuve a contrario dans le fait que les sociétés behringiennes, riches économiquement et démographiquement, voyaient au contraire les vendettas dégénérer en véritables guerres intra - et inter-tribales ${ }^{2}$.

Au vu de ces différents exemples, nous devons donc constater à la fois l'unicité et la diversité des causes de la vengeance familiale. Globalement, elles se rapportent à deux : les femmes, le meurtre. Mais, dès qu'on se penche sur les exemples concrets, on s'aperçoit que ce principe est susceptible d'amodiations régionales : chez certaines populations, le meurtre ou le rapt de la femme n'entraîne pas automatiquement la vengeance. Nous avons proposé des ébauches de solution, en ayant bien conscience de leur caractère très hypothétique. Il reste qu'à notre sens, cette diversité n'est que le reflet de celle des sociétés inuit, qui vivent dans des contextes historiques, économiques, climatiques et socio-politiques différents ${ }^{3}$. L'anormal serait donc qu'on ne constate pas également des divergences dans leurs règles juridiques, lesquelles sont, comme chacun le sait, le reflet des tensions socio-politiques et du milieu économique qui les engendrent. L'historien des sociétés à "histoire ouverte" saisit d'ailleurs diachroniquement cette réalité. En ce qui concerne les sociétés inuit - donc, de toute façon, l'histoire n'est pas "close", comme on l'a trop souvent répété - c'est dans l'espace que nous pouvons saisir cette action des structures socio-économiques sur le juridique.

Nous allons d'ailleurs en avoir d'autres exemples en étudiant les modalités de la vengeance familiale.

Cf. E. ADAMSON-HOEBEL, op. cit., 87.

Cf. J. MALAURIE, op. cit., (Raids et esclavage), 5, 6.

Sur la pluralité des sociétés inuit, cf. D. DAMAS, "The Diversity of Eskimo Societies", dans Man, the Hunter (Chicago, 1968), 111-117. Sur la nécessité d'une approche comparative interne des sociétés inuit, cf. N. ROULAND, op. cit. (L'ethnologie juridique des Inuit). 
[p. 56]

\section{B) LES MODALITÉS}

$\underline{\text { Retour à la table des matières }}$

Nous traiterons brièvement ici de trois problèmes : l'ordre des vengeurs et celui des individus objet de la vengeance ; la façon dont est exécutée la vengeance ; les effets annexes de la vengeance ${ }^{1}$.

1) L'ordre des vengeurs. Les vengeurs sont exclusivement les parents mâles : la femme est frappée d'une incapacité du point de vue du droit de vengeance ${ }^{2}$. Il s'agit des plus proches parents, mais leur distribution varie suivant les groupes considérés ${ }^{3}$. Le fils vient en premier au Groënland, au Labrador, en Alaska. En Alaska, si le fils n'est pas majeur, on différera la vengeance jusqu'à ce qu'il soit en mesure de l'accomplir ; en cas d'absence de fils ce sera, par ordre successif : frère, père, oncle ${ }^{4}$. Pour notre part, nous avons noté que ce sont les frères chez les Inuit Chugach ${ }^{5}$; des contes Iglulik font également allusion au frère ${ }^{6}$, mais rien, à vrai dire, ne permet d'en déduire une règle générale ; chez les Nunamiut (Alaska) ${ }^{7}$, le devoir de vengeance incombe aux enfants ; chez les Nunivak (Nord-Ouest Alas$\mathrm{ka}$ ), en principe les vengeurs n'appartiennent pas à la même génération que la victime, qui doit être vengée par son fils aîné, qu'on élève dans cette idée ${ }^{8}$. (On notera que dans certains cas, l'appartenance d'un même individu aux deux groupes en conflit l'excepte de la participation à la vengeance. Ainsi, chez les Inuit Arviligjuarmiut - qui appartiennent aux Inuit Netsilik de Pelly Bay - le membre de deux ilagiit en querelle ne doit pas participer au conflit ${ }^{9}$ ).

[p. 57]

- Chez les Inuit Centraux, le fils est, en revanche, absent de l'ordre des vengeurs et remplacé par les frères, le père ou les neveux de la victime ${ }^{10}$.

Pour plus de détails, cf. H. KUNIG, op. cit. (Der Rechtsbruch 510-514.

Cf. H. KONIG, op. cit., 510, 777.

Ibid., 510-512.

Ibid., 511.

Cf. K. BIRKET-SMITH, op. cit. (The Chugach Eskimo), 97.

Cf. K. RASMUSSEN, op. cit. (Intellectual culture of the Iglulik Eskimo), 297-299.

Cf. N.J. GUBSER, op. cit., 151.

Cf. M. LANTIS, "The social culture of the Nunivak Eskimo", dans Transactions of the American Philosophical Society XXV-3 (1946), 250.

9 Cf. A. BALIKCI, "Development of basic socio-economic units in two Eskimo communities", dans National Museum of Canada Bulletin, 202 (Ottawa, 1964), 27.

10 Cf. H. KÖNIG, op. cit.., 510. 
Il est difficile d'expliquer ces disparités régionales : les causes économiques ne paraissent pas ici déterminantes. En effet, on pourrait penser que chez les populations pauvres, on préférerait prendre comme vengeurs les membres de la même génération que la victime, afin d'épargner les futurs adultes, sur lesquels repose la survie du groupe. Mais ce raisonnement est démenti par les faits : si chez les Inuit centraux le fils est bien absent de l'ordre des vengeurs, en revanche il apparaît en Alaska ... Et, de toute façon, le point de départ de l'argumentation est incertain, puisque le groupe souffrira également de la perte d'adultes décimés par la vengeance.

On rencontre le même genre de problème quant à l'ordre de ceux qui sont susceptibles d'être atteints par la vengeance : au premier chef, bien entendu, est visé le coupable. Mais, à défaut, et au titre de la responsabilité collective, ses parents, et en priorité ses descendants : enfants, petits-enfants, et seulement après : frères et autres parents. (Si le coupable est une femme, elle sera objet de la vengeance, mais il ne semble pas qu'elle puisse être atteinte en tant que parente ${ }^{1}$ ). Cet ordre "privilégie" donc les parents issus du meurtrier : H. König en voit la raison dans le fait que les vengeurs veulent sanctionner ceux qui vivaient dans l'entourage du meurtrier au moment du crime ${ }^{2}$. Cette raison ne semble pas convaincante : les ascendants du meurtrier peuvent avoir, eux aussi, continué à cohabiter avec lui ; quant à ses descendants, ils peuvent l'avoir quitté, notamment en cas de mariage.

2) Les modalités d'exécution. Nous avons déjà vu que, dans certains cas, la vengeance pouvait être différée de plusieurs années ${ }^{3}$, notamment quand elle incombe au fils aîné et que celui-ci est encore en [p. 58] bas âge. Nous avons également noté que ce retard était déjà, au niveau psychologique, constitutif d'une partie de la sanction ${ }^{4}$.

Quant aux modalités concrètes, c'est, dans la plupart des cas, par surprise qu'est exercée la vengeance ${ }^{5}$ : au cours d'une expédition de chasse ; la nuit, durant le sommeil de la victime. Le coup est d'ailleurs en général porté dans le dos. Cependant, là encore, ce principe général souffre des exceptions :

- Sur la côte ouest du Groënland, le vengeur, avant de frapper, doit donner les motifs de son acte ${ }^{6}$.

- Chez les Inuit Netsilik, la vengeance est mise en œuvre selon des procédés destinés à limiter les pertes en vies humaines. Quand un individu est considéré

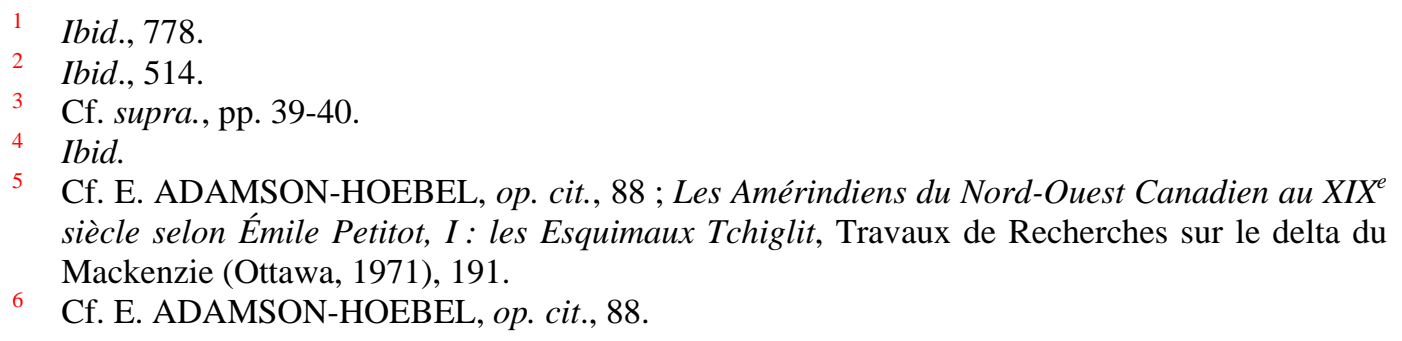


comme dangereux, ses compagnons de chasse discutent de l'affaire, puis, à l'hiver, entreprennent la vengeance. Généralement, ils sont apparentés, mais des nonparents peuvent se joindre au groupe. Lorsque d'un côté, sur une dizaine d'individus, trois ou quatre sont blessés ou morts, les survivants rentrent chez eux. Les vieux ne sont jamais attaqués. La résidence influe sur la participation à la vengeance : si un individu originaire de $A$ va vivre en $B$, et accompagne une expédition de A contre $\mathrm{B}$, les habitants de $\mathrm{A}$ le lui reprocheront ${ }^{1}$.

- Chez les Inuit Caribou (Padleïmiut) les incidences des conflits sont aussi limitées par le recours à des duels de héros : au bout de deux ou trois morts, les pourparlers de paix s'engagent ${ }^{2}$. La surprise n'apparaît pas non plus la règle générale chez les Caribou ${ }^{3}$. D'ailleurs, d'une façon générale, les cas de mise en œuvre de la responsabilité [p. 59] collective sont rares chez les Caribou ${ }^{4}$, (ainsi que chez les Netsilik ${ }^{5}$ ) et les conflits sont réglés avec le plus de discrétion possible. (Dans la même optique, on se souvient que lorsque les Caribou ostracisent un perturbateur, ils l'abandonnent par surprise plutôt qu'ils ne l'exilent ${ }^{6}$.

En ce qui concerne les Caribou, il paraît possible d'expliquer, une fois de plus, l'originalité de leurs solutions par la faible marge de survie dont dispose chez eux la communauté : très restreinte démographiquement et constamment à la limite de la survie, elle ne peut se permettre de subir les effets meurtriers des vendettas, et tend donc à limiter au maximum leur éventualité.

3) Effets annexes de la vengeance. Par la perturbation qu'elle cause dans les structures des familles considérées, la vengeance entraîne une série d'effets annexes chez certaines populations inuit :

- Une fois la vengeance assouvie, la famille de la victime, et plus particulièrement sa femme et ses enfants se retrouvent sans protection, et dans l'incapacité de subsister. Dans la plupart des cas, et même quand la femme ne se situait pas à la source de la vendetta, le meurtrier doit veiller sur la femme et les enfants de sa victime ${ }^{7}$.

\footnotetext{
Cf. G. VAN DEN STEENHOVEN, "Un récit historique du "bon vieux temps" chez les Esquimaux Netsilik", dans Eskimo, 61 (mars-juin 1962).

Cf. J. MALAURIE, op. cit., (Les derniers rois de Thule), 180-181.

K. RASMUSSEN (Intellectual Culture, 106-107) cite un cas dans lequel les vengeurs viennent avertir le père adoptif de leur victime présumée.

4 Cf. J. GABUS, op. cit.., 140, 142 ; G. VAN DEN STEENHOVEN, op. cit. (Caribou Eskimo legal concepts...), 6.

Cf. G. VAN DEN STEENHOVEN, ibid., 48, 55.

Cf. supra, p. 44.

Cf. E. ADAMSON-HOEBEL, op. cit., 87.
} 
- En Alaska, la vengeance concerne, non seulement la famille des parties en cause, mais également les partenaires d'échange et leurs familles ${ }^{1}$. Mais l'institution d'échange de conjoints peut, en sens inverse, limiter l'étendue de la vengeance : à Point Barrow (Alaska) si certains individus appartenant à deux familles en lutte au titre de la vengeance font échange de leurs femmes, les enfants issus de ces unions n'exerceront plus entre eux la vengeance ${ }^{2}$.

- Chez les Inuit du Nord de l'Alaska, la revanche sanglante entre [p. 60] deux familles entraîne la suspension des pratiques de partage et de vie communautaire ${ }^{3}$.

Notons enfin, pour terminer, que la vengeance sanglante familiale n'est pas systématique : il est des cas dans lesquels elle ne suit pas la survenance des faits qui en sont habituellement les motifs. C'est chez les Caribou ${ }^{4}$ et les Inuit du Cuivre ${ }^{5}$ que les auteurs font le plus fréquemment mention d'une telle absence de réaction. Les raisons de cette abstention peuvent être diverses : les vengeurs ne se sentent pas assez forts pour exercer des représailles, la famille de la victime estime que le meurtre a été commis à bon droit ${ }^{6}$, les individus impliqués sont liés par des échanges de femmes, etc. Il n'est pas sans importance que ce soit chez les Caribou et les Inuit du Cuivre que soit souvent signalée cette absence de réaction : nous avons déjà dit combien leur contexte démo-économique imposait à ces populations une limitation maximale des conflits.

De toute façon, l'absence éventuelle de vengeance familiale ne signifie nullement, comme le voudrait G. Van den Steenhoven pour les Inuit Caribou et Netsilik ${ }^{7}$, qu'il y ait chez ces populations absence de droit ou, à tout le moins, "rudimentary law in a primitive anarchy" (197) ${ }^{8}$. En effet :

- Si, dans la définition du droit, l'intention répétitive est bien fondamentale, il ne faut pas la confondre avec la survenance automatique de la sanction ${ }^{9}$.

1 Cf. L. HENNIGH, "Functions and limitations of Alaskan Eskimo Wife Trading", dans Arctic, XXIII-I (1970), 28.

2 Cf. A.J. RUBEL, "Partnership and wife-exchange among the Eskimo and Aleut of Northern North America", dans Anthropological Papers of the University of Alaska, X-1 (1961), 63. Certaines relations pré-existantes à la vengeance entre des membres des deux groupes familiaux en lutte peuvent également exclure ces individus de la participation à la revanche (Cf. $\mathrm{H}$. KÖNIG, op. cit.) Cf. R.F. SPENCER, op. cit., 72.

Cf. G. VAN DEN STEENHOVEN, op. cit., (Caribou Eskimos legal concepts...)

Cf. D. JENNESS, The life of the Copper Eskimos, Rep. of the Canadian Arctic Exp. 1913-18, XII (Ottawa, 1922), 95 ; G. VAN DEN STEENHOVEN, op. cit., (Legal Concepts), 48, 55.

Cf. E. ADAMSON-HOEBEL, op. cit., 85.

Cf. G. VAN DEN STEENHOVEN, op. cit., (Caribou Eskimo Legal Concepts 4, 6 ; Legal Concepts..., 62.

8 Refusant ce terme de "rudimentary law" aux Caribou, G. VAN DEN STEENHOVEN le concède aux Netsilik, op. cit., (Legal Concepts...), 63.

9 Cf. supra, pp. 16, 18. 
[p. 61]

- La crainte de la sanction est déjà une sanction ${ }^{1}$.

- A. Balikci fait remarquer, à juste titre, que les cas concrets sur l'analyse desquels G. Van den Steenhoven se base, pour appuyer son affirmation, mettaient en jeu des individus qui étaient des compagnons de camp, et que la communauté n'aurait pu tolérer entre eux une vengeance qui l'aurait mise en péril : les groupes exerçant la vendetta sont, chez les Netsilik, relativement distants au point de vue de leur résidence ${ }^{2}$.

- Enfin, on ne peut s'empêcher de prendre, une fois de plus, G. Van Den Steenhoven en flagrant délit d'ethnocentrisme. Pour corroborer son assertion sur la faiblesse des structures juridiques, l'auteur écrit que la notion de justice est également absente chez les Inuit Netsilik :

"For murder disturbs its 'peace', and it is the community's concern that this 'peace' be maintained, rather than justice - which as an abstraction is unknown to them" ${ }^{3}$.

C'est tout simplement oublier que, chez les populations archaïques, le terme de justice, pour recevoir une acception différente de celle que nous lui accordons dans nos sociétés, n'en est pas moins réel. ${ }^{4}$

On voit donc tout l'intérêt qui s'attache à une lecture comparative des institutions juridiques et judiciaires des sociétés inuit. À notre sens, seule une telle optique peut rendre intelligible les diversités et les disparités coutumières qu'on rencontre dans l'application des principes généraux. Dès qu'on entend atteindre un certain niveau de précision, il est, en effet, illusoire de parler d'un droit inuit, de même qu'il est vain de parler d'une société inuit ${ }^{5}$.

$$
\text { [p. 62] }
$$

L'historien du droit français comprendra d'ailleurs fort bien cette nécessité : que penserait-il, en effet, d'un auteur qui, prétendant étudier le droit médiéval coutumier, ne distinguerait pas entre les grands groupes géographiques de coutumes territoriales ?

\section{Cf. supra, pp. 16-17.}

Cf. A. BALIKCI, op. cit., 184.

G. VAN DEN STEENHOVEN, op. cit., (Legal concepts), 48. L'auteur reconnaît, d'ailleurs, dans une autre étude que "...en ce qui concerne la vengeance sanglante, en l'absence de toute autorité constituée, ces exécutions correspondaient à une certaine conception de la justice". (G. VAN DEN STEENHOVEN, op. cit., (Un récit historique du "bon vieux temps").

Cf. supra, pp. 13-14, 17.

C'est ce qu'a fort bien mis en relief J. Malaurie dans son étude sur les civilisations esquimaudes, op. cit., (Essai d'anthropogéographie). Cf. également du même auteur : Spécificité des sociétés esquimaudes, (op. cit.), 10-11. 
Les remarques que nous avons faites, dans ce sens, dans les lignes qui précèdent nous ont permis de montrer que, chez les populations inuit les plus précaires et les plus archaïques, des limitations étaient apportées à la vengeance familiale pour éviter une trop grande perturbation causée à l'équilibre de la société globale. Nous allons voir maintenant qu'en effet le groupe dispose de plusieurs moyens d'intervention plus ou moins directe dans ce mode privé de résolution des conflits pour éviter qu'il ne dégénère dans la disparition du groupe en son entier par l'enchaînement ad libitum des vendettas.

\section{C) LES LIMITATIONS APPORTÉES PAR LA COMMUNAUTÉ À LA VENDETTA}

\section{$\underline{\text { Retour à la table des matières }}$}

Le danger que nous signalions plus haut pour la communauté de se voir détruite par l'enchaînement des vendettas ${ }^{1}$ n'est pas théorique :

- Chez les Nunamiut (Alaska), aux niveaux de stratification sociale supérieurs à la bande, les vengeances se succèdent durant plusieurs générations car il n'existe aucune autorité politique supérieure capable de les limiter ou d'y mettre fin. En revanche, dans les groupes dirigés par une autorité (la bande et les groupements familiaux), la vengeance, quand elle survient, entraîne la désagrégation de la communauté ${ }^{2}$ et, en temps normal, tout est mis en œuvre pour empêcher son déclenchement par le leader du groupe familial ou l'umialik de la bande.

- Les Inuit de la Côte Est du Groënland étaient particulièrement sensibles aux dangers de la vendetta familiale eu égard à la faible taille démographique de leurs groupes, et disaient qu'en raison de ce dernier facteur les crimes devenaient de plus en plus fréquents, et que la proportion des vengeurs et des vengés allait croissant par rapport à l'ensemble de la population ${ }^{3}$.

[p. 63]

- J. Malaurie rapporte qu'en un lieu nommé Inouarfigssouaq (la baie du Grand Massacre) (Terre d'Inglefield, Nord-Ouest du Groënland), un grand-père intervint pour séparer deux enfants qui se battaient. Malencontreusement, il tue l'adversaire de son petit-fils. Aussitôt, les familles interviennent, et la tuerie devient générale, si bien que toute la population du village fut anéantie ${ }^{4}$.

\footnotetext{
1 "The feuds are said to have gone on endlessly, being remembered after several generations" (R.F. SPENCER, op. cit., 72).

Cf. L. POSPISIL, op. cit., (Law and societal structure...) 427.

Cf. K. RASMUSSEN, op. cit., (Life and doings of East Greenlanders...), 89.

Cf. J. MALAURIE, op. cit., (Les derniers rois de Thule), 408-409.
} 
Ces dangers, résultant de l'enchaînement des vendettas ont été perçus par bon nombre de sociétés qui les pratiquaient. Pour reprendre l'exemple de la Rome royale ${ }^{1}$, le roi intervient pour éviter la mise en œuvre de la vengeance privée tant que le bon droit de celui qui veut l'exercer n'est pas établi, et n'autorise cette vengeance que dans la mesure où elle revêt des formes codifiées et juridiques. Petit à petit, le roi limite les cas de vengeance privée et, en tant qu'autorité politique souveraine, oblige les particuliers à s'adresser à lui ${ }^{2}$. On retrouve le même genre de processus dans le Moyen-Âge français : progressivement le roi limite les recours à la guerre privée et impose sa justice ${ }^{3}$.

Il paraît donc que les sociétés archaïques, à quelque époque qu'elles se situent, et à des degrés divers, aient connu des mécanismes conçus pour limiter - ou éviter - le recours à la vendetta ${ }^{4}$.

Nous allons étudier plusieurs de ces limitations pratiquées par les sociétés inuit.

Auparavant, on observera que la fixation coutumière des modalités de la vengeance est déjà en elle-même une intervention du groupe : cette vengeance, en effet, ne s'exerce pas de n'importe quelle façon (ordre [p. 64] des vengeurs, place particulière de la femme, etc.), et les coutumes qui la règlementent sont l'expression d'un groupe qui inclut et dépasse les familles : la communauté du camp ${ }^{5}$.

La vendetta familiale ne dégénère pas forcément : elle peut s'arrêter avec la mort du coupable. Pour A. König, elle prend ainsi fin dans la plupart des cas ${ }^{6}$. Dans le même sens, R.F. Spencer note, pour les Inuit du Nord de l'Alaska, que la vengeance sanglante peut très bien se conclure rapidement, mais que son degré d'extension dépend du niveau auquel elle se déroule : à l'intérieur d'une même

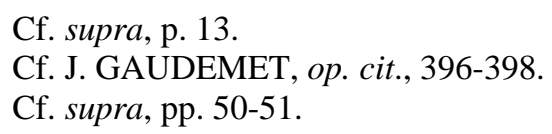

(Dans le même sens, cf. A. DORSINFANG-SMETS, op. cit., 18). Dans les tribus dotées d'une organisation socio-politique formalisée, le recours à la vendetta est proscrit : cf. E. ADAMSON-HOEBEL, op. cit., 330. Pour G. SAWER, op. cit., 40, la vengeance se répétant à l'infini est presque inconnue dans les sociétés les plus archaïques, où un seul acte de revanche mettrait fin au conflit. Dans l'éventualité où, malgré tout, la situation échapperait au contrôle des belligérants, il y aurait dans tous les cas une forme de réponse sociale pour restaurer la paix... Ce point de vue paraît quelque peu systématique.

5 "Elle (la vendetta familiale) marque seulement une étape où l'État n'a pas encore assez d'autorité pour prendre lui-même en main la fonction répressive, mais cela ne veut pas dire qu'elle soit laissée à l'arbitraire du groupe lésé. La coutume joue ici un rôle qui n'a pas été suffisamment reconnu. Expression d'un groupe social plus étendu, elle règlemente impérativement la répression familiale, désigne le vengeur de sang, flétrit les membres du groupe qui s'abstiendront de prendre part à l'expédition punitive. Bref, si l'exécution du châtiment est, dans une mesure variable, laissée aux parents de la victime, la répression elle-même est l'affaire de la collectivité et non de l'individu, et ne saurait dépendre de son caprice". (H. LEVY-BRUHL, op. cit., 11671168)

6 Cf. H. KÖNIG, op. cit., 786. Le rachat par composition pécuniaire semble inconnu des Inuit (ibid., 792), à la différence du Wehrgeld germain. 
communauté, le conflit peut être rapidement réglé et oublié ; en revanche, lorsqu'il survient entre deux communautés, il peut durer beaucoup plus longtemps ${ }^{1}$. La raison en est, certainement que, dans le premier cas, l'unité du groupe exige une fin rapide des conflits internes, toujours susceptibles de mener à la dislocation.

Cependant, la vendetta familiale est loin de se dérouler toujours suivant ces modèles idéaux. Aussi le groupe est-il parfois obligé d'intervenir de façon directe pour en limiter les effets perturbateurs ${ }^{2}$. Nous pouvons citer une série d'exemples de ces interventions :

o Le groupe peut entériner le meurtre s'il l'estime avoir été commis a bon droit : c'est donc une des modalités pour le groupe d'exercer une action judiciaire indirecte.

[p. 65]

- $\quad-$ G. Van den Steenhoven rapporte que, chez les Inuit Netsilik de Pelly Bay, si le meurtrier est un bon chasseur et si son acte paraît au groupe suffisamment justifié, ce dernier n'agit nullement pour le sanctionner, même si la famille de la victime ne se manifeste pas ${ }^{3}$.

- Chez les Inuit Caribou, si la communauté donne raison au justicier du meurtrier, il n'y a pas de vendetta familiale ${ }^{4}$.

o Mais souvent, il ne suffit pas au groupe d'entériner l'acte de revanche ou le meurtre pour que la vendetta s'arrête. Il doit intervenir de façon plus active :

- Le shaman peut décréter un échange de femmes ${ }^{5}$

- Plus souvent, le groupe va mandater un de ses membres : la communauté se réunit et décide l'exécution des membres d'un des groupes familiaux en cause ${ }^{6}$. Dans ce dernier cas, il est intéressant de remarquer que :

- Le groupe éliminé n'est pas forcément celui qui est dans son bon droit, mais celui qui dispose d'un moins grand prestige économique et social sur le reste de la bande. Autrement dit, comme nous l'avons déjà maintes fois constaté à propos des sociétés Inuit, le conflit est résolu, non pas tant par l'appréciation des responsabilités que par la prééminence accordée au maintien de l'unité du groupe. Laquelle sera assurée par la survivance du groupe familial qui contribue le plus à son unité.

Cf. R.F. SPENCER, op. cit., 98.

À cet égard, l'affirmation de E. WEYER paraît très exagérée : "The tribe or community is not consulted in the enforcement of blood vengeance ; it devolves upon the family or the kingroup" (E.M. WEYER, The Eskimos (Archon Books, 1969), 222).

Cf. G. VAN DEN STEENHOVEN, op. cit., (Legal concepts), 48.

Cf. J. GABUS, op. cit., 142.

Cf. L. GUEMPLE, op. cit., 101 (Nous avons déjà vu (cf. supra., p. 59 qu'à Point Barrow (Alaska) l'échange de conjoints peut limiter les effets de la vengeance.

6 Ibid. 
- Les exécuteurs de la sentence du groupe n'agissent pas en tant que personnes privées, mais comme mandataires du groupe : la preuve en réside dans le fait qu'ils sont pris parmi les plus proches parents du groupe à éliminer ${ }^{1}$, ce qui évite toute éventualité de résurgence ultérieure de la vendetta. Ces exécuteurs peuvent, également, être les [p. 66] personnalités les plus marquantes du groupe, qui agissent après avoir obtenu l'accord de la communauté ${ }^{2}$.

Cependant, il peut arriver que le groupe familial, objet de la sentence du groupe, ne l'accepte pas et continue la vendetta. Ainsi, V. Stefansson rapporte qu'à Point Atkinson, en 1880, un homme s'était rendu si intolérable par ses agissements que la communauté décida de s'en débarrasser. Trois des hommes les plus énergiques et les plus dévoués au groupe furent désignés comme exécuteurs et l'un d'entre eux poignarda effectivement le perturbateur. Mais la famille de ce dernier n'accepta pas de voir en cette exécution la fin du conflit et fit savoir qu'elle allait tuer les trois exécuteurs mandatés par le groupe. En l'espace de quatre ans, elle réussit à en tuer deux, et le troisième n'échappa à sa vengeance qu'en raison d'une épidémie qui décima cette famille ${ }^{3}$. On remarquera qu'ici nous avons à faire à un véritable acte de rébellion contre l'autorité du groupe ${ }^{4}$.

- Ce n'est pas tant la vengeance familiale qui se poursuit que le groupe qui est attaqué, dans la personne de ceux qu'il avait mandatés pour mettre fin au conflit.

- Ce point est confirmé par le fait que les trois individus désignés par le groupe sont visés par la vengeance, alors qu'en pratique un seul d'entre eux a exécuté la décision collective de liquidation physique du perturbateur.

o Il existe certaines procédures qui, tout en laissant le soin de leur mise en œuvre à l'initiative privée des parties en cause, n'en manifestent pas moins, de façon évidente, l'intervention du groupe par le biais de la coutume pour limiter l'étendue de la vengeance privée au nom de l'intérêt public :

- Chez les Inuit centraux, le meurtrier va vivre parmi les parents de sa victime. Au bout de quelques semaines l'un d'entre eux le provoque à une rencontre de lutte : si le meurtrier perd, il est mis à mort, s'il [p. 67] est vainqueur il peut tuer un des parents de sa victime ${ }^{5}$. Dans les deux cas, la vengeance s'arrête là.

- Chez les populations behringiennes "on signale des cas de transaction entre les parties ; la famille du meurtrier s'offrant à remplacer la victime ; traité en esclave, il sera le mari de la veuve et père de ses enfants" ${ }^{6}$. Ce dernier cas n'est pas sans rappeler le régime de l'abandon noxal en droit romain, qui remonte aux

Ibid. Dans le même sens, cf. J.W. LAPIERRE, op. cit., 494, n. 246.

Cf. J.W. LAPIERRE, op. cit., 50 et n. 246 ; v. STEFANSSON, op. cit., 365.

Cf. V. STEFANSSON, op. cit., 365-366.

Pour un cas similaire, cf. infra, pp. 73-74 (cas d'Aqsuq, de Bathurst Inlet).

Cf. A.S. DIAMOND, L'évolution de la loi et de l'ordre, (Paris, 1954), 81.

J. MALAURIE, op. cit., (Raids et esclavage), 5. 
plus anciennes conceptions des Romains en matière de délits privés ${ }^{1}$ et, à ce titre, le chef de famille du délinquant pouvait l'abandonner à la vengeance de sa victime et de ses parents, qui le réduisait à une condition quasi servile. Mais cet abandon constituait la solution du conflit.

o Enfin, dans certains cas marginaux, la vengeance sanglante peut être limitée par un effet d'acculturation : K. Rasmussen rapporte qu'à Ammassalik (Côte Est du Groënland), un meurtre ne fut pas vengé par la famille de la victime, probablement parce que ses parents avaient été baptisés et par peur de la justice des Blancs ${ }^{2}$.

En conclusion, on fera observer, une fois de plus, que si les règles énoncées dans les lignes qui précèdent constituent bien des normes juridiques, il faut se garder de croire en leur application systématique et générale : nous avons vu que, dans certains cas, les individus ou les groupes familiaux se rebellent contre la coutume $^{3}$. D'autre part, et surtout, le recours à la vengeance sanglante n'est pas d'une égale fréquence dans toutes les sociétés Inuit : là encore ${ }^{4}$, tout [p. 68] dépend du contexte socio-économique. Ainsi, elle semble inconnue chez les Inuit Iglulik $^{5}$. Dans les sociétés précaires de l'Arctique central et oriental, la vendetta familiale est relativement peu fréquente, et implique rarement toute une bande, car les relations sociales sont atomistiques et les querelles tendent à demeurer très circonscrites ${ }^{6}$. (Nous avons d'ailleurs vu que ces populations mettaient en œuvre des coutumes propres à éviter ou limiter la vendetta ${ }^{7}$ ). En revanche, chez les riches et démographiquement fortes populations behringiennes, la vendetta dégénère en guerres intra et inter tribales ${ }^{8}$. Il y a donc bien une anthropo-géographie juridique des sociétés inuit. Nous allons retrouver ces mêmes diversités coutumières régionales en étudiant, dans une seconde section, l'intervention directe du groupe dans le règlement des conflits.

1 Sur le régime de la noxalité et le fonctionnement des actions noxales, cf. J. MACQUERON, Histoire des obligations : le droit romain (Publications du Centre d'histoire institutionnelle et économique de l'Antiquité classique, Série Mémoires et Travaux, no 1 (Aix-en-Provence, 1975), 325-331.

Cf. K. RASMUSSEN, op. cit., (Life and doings of East Greenlanders...) 88.

Cf. supra, p. 66.

Cf. supra, pp. 61-62.

Cf. E.M. WEYER, op. cit., 222.

Cf. L. GUEMPLE, op cit., 101.

Cf. supra, p. 62 sq.

Cf. J. MALAURIE, op. cit. (Raids et esclavage), 5, 6. 


\section{Section II : MODALITÉS DE L'ACTION PUBLIQUE : INTERVENTION DIRECTE DU GROUPE DANS LE RÈGLEMENT DES CONFLITS}

\section{Retour à la table des matières}

En droit positif français, le terme d'action publique désigne la forme juridique et judiciaire que prend la réaction de la société à l'accomplissement de toute infraction à la loi pénale par un de ses membres. Cette action a pour but la répression de l'atteinte portée à l'ordre social et pour objet l'application d'une peine ou d'une mesure de sûreté au délinquant, et est exercée au nom de la société par ses représentants institutionnels. Elle diffère de l'action civile, qui a pour but d'obtenir la réparation du préjudice éprouvé par la victime ${ }^{1}$. Il va de soi qu'une comparaison trop poussée avec les modalités d'intervention du groupe dans le règlement des conflits chez les sociétés inuit [p. 69] serait vaine et ethnocentrique ${ }^{2}$. Cependant, il nous a paru légitime de regrouper ces processus d'intervention sous le nom d'action publique. En effet, nous y voyons clairement le groupe se manifester, et imposer au nom de l'intérêt public, des procédures de règlement qui ont avant tout pour but la défense sociale, l'équilibre et la survie du groupe, et ce au-delà même de la réparation du préjudice subi par une des parties en cause ; le but essentiel est la restauration de la paix sociale, plus que la détermination des responsabilités ${ }^{3}$. Ces traits fondamentaux nous paraissent suffisants pour être autorisé à parler d'une action publique légale ${ }^{4}$. Cependant, G. Van den Steenhoven nie que la décision de la communauté de se débarrasser d'un perturbateur ait un caractère judiciaire : il s'agirait d'une simple vengeance, n'impliquant nullement la mise en œuvre de procédés destinés à faire justice ${ }^{5}$. Nous ne pouvons ici que renvoyer le lecteur à nos précédentes observations montrant l'ethnocentrisme de telles affirmations ${ }^{6}$, et noter, avec M. Gluckmann, que le fait que les parents de l'homme

1 Sur ces notions générales, cf. G. STEFANI - G. LEVASSEUR, Procédure pénale (Paris, 1966), 71-72.

2 Pour un aperçu général de ces modes d'intervention, cf. H. KÖNIG, op. cit., (Der Rechtsbruch), 302-311 ; E.M. WEYER, op. cit., 224-228.

3 Cf. BIRKET-SMITH, op. cit., (Mœurs et coutumes), 177.

4 Dans le même sens, RADCLIFFE-BROWN considérait que l'emploi de ces termes était justifié dans la mesure où un certain nombre d'hommes expérimentés peut, dans une société donnée, déclarer indésirable un perturbateur et décider son exécution (Cf. M. GLUCKMANN, op. cit., 116). Dans le même sens, cf. aussi A. DORSINFANG-SMETS, op. cit., 19.

5 Cf. G. VAN DEN STEENHOVEN, op. cit., (Leadership and law...) 116. Les mêmes remarques peuvent être formulées contre l'appréciation - identique - de K. BIRKET-SMITH, op. cit., (Mœurs et coutumes), 140.

6 Cf. supra, pp. 12-14. 
dont la communauté a décidé de se débarrasser soient consultés avant la mise en œuvre de la sanction donne déjà un caractère judiciaire à cette procédure ${ }^{1}$.

L'expulsion ou la liquidation physique du perturbateur constitue donc la première modalité selon laquelle le groupe exerce l'action publique. Mais il peut aussi apporter aux conflits une solution moins [p. 70] brutale, d'aspect psychothérapeutique, où il intervient par une série de formes très codifiées, institutionnelles, et en exerçant une véritable juridiction : il s'agit des duels judiciaires. Nous étudierons successivement chacune de ces questions.

\section{A) LA DÉCISION COMMUNAUTAIRE DE LIQUIDATION PHYSIQUE DE L'ENNEMI PUBLIC}

$\underline{\text { Retour à la table des matières }}$

En droit positif français, la notion criminologique d'état dangereux se définit juridiquement essentiellement par :

- Un élément matériel : " ... la conviction qu'une infraction future est très probable doit reposer sur des faits antérieurs précis et contrôlables sur lesquels s'échafaudent ensuite le raisonnement et les prévisions, et dont le juge puisse faire état dans sa sentence" ${ }^{2}$.

- Un élément légal : "l'élément matériel ainsi précisé doit être contenu dans un texte qui constituera ainsi une sorte d'élément légal de l'incrimination de l'état dangereux, afin que l'individu ait pu savoir à l'avance que son comportement risquait d'autoriser la recherche d'un état dangereux éventuel susceptible de conduire, en cas de constatation, à une mesure de sûreté contre lui" ${ }^{3}$.

Mutatis mutandis, on peut, comme pour la définition de l'action publique ${ }^{4}$, retrouver certains aspects de ces principes dans le droit traditionnel inuit. En effet, pour que la communauté entreprenne une action d'exécution contre un perturbateur, celui-ci doit apparaître comme un ennemi public. Son état dangereux pourrait ainsi se définir :

- Élément légal : il ne faut évidemment pas le chercher dans un texte, puisque nous sommes dans des sociétés de droit oral, mais dans la coutume. Chaque

\footnotetext{
"I suggest that even a proto-judicial process is found only when evidence is examined and assessed (...). Usually, as among Eskimo, the consent of the villain's kin must be secured in order to exclude blood-revenge. This is definitely a proto-judicial process, involving rational discussion of the case in the light of sensory evidence" (M. GLUCKMANN, op. cit., 190).

Cf. G. STEFANI - G. LEVASSEUR, op. cit. (Droit Pénal...) 103.

Ibid., 113.

Cf. supra, p. 68.
} 
individu sait que la répétition de certaines infractions l'amènera tôt ou tard à un affrontement avec le groupe, d'autant plus que ce dernier, comme on le sait, essaie auparavant de l'amener à s'amender par divers procédés que nous avons énumérés plus haut.

- Élément matériel : le perturbateur doit :

- Soit avoir commis à plusieurs reprises des infractions graves laissant présager à la communauté qu'il est susceptible d'en commettre [p. 71] de nouvelles dans l'avenir. Au premier rang de ces délits, le meurtre ${ }^{1}$. Mais, également, les violations graves des règles de vie communautaire : les cruautés et mauvais traitements ${ }^{2}$; le mensonge et la paresse persistants ${ }^{3}$, les actes censés compromettre le succès de la chasse ${ }^{4}$, le refus du partage collectif du gibier ${ }^{5}$, l'adultère répété ${ }^{6}$. En ce qui concerne ces infractions, l'exécution n'a donc lieu qu'en cas de récidive.

- Soit s'être rendu coupable d'infractions mettant directement en jeu la survie de la communauté : il s'agit donc d'une catégorie d'infractions qu'on peut ranger sous la rubrique de délits publics, c'est-à-dire de délits mettant en jeu l'ordre social, justifiant la réaction de la société par la voie de l'action publique, et l'application d'une peine au coupable ${ }^{7}$. Ces infractions sont essentiellement :

a) La sorcellerie ${ }^{8}$ : dans toutes les sociétés archaïques, c'est un crime particulièrement grave ${ }^{9}$ car il perturbe les équilibres fondamentaux entre le monde concret et les formes surnaturelles. Il va de soi que les shamans sont particulièrement vigilants quant à ce genre d'infractions. Mais eux-mêmes peuvent être condamnés à mort par le groupe s'ils abusent de leurs pouvoirs ${ }^{10}$.

b) Les délits "politiques" : la mort sanctionne l'individu qui, dépassant les limites du leadership autorisé, essaie d'instaurer à son [p. 72] profit un pouvoir tyrannique; le refus de distribution au groupe de leurs richesses par les individus qui ont pu en amasser ${ }^{11}$.

1 Cf. H. KÖNIG, op. cit., 309. B. SALADIN D'ANGLURE, op. cit., 188, note à propos des Inuit de Kangirsujjuaaq (Nouveau-Québec),que lorsque le meurtre est commis avec des circonstances atténuantes (légitime défense, infanticide, et sénilicide, vengeance familiale, réaction à des actes de sorcellerie), il n'entraîne pas la réaction de la communauté.

Cf. E. ADAMSON-HOEBEL, op. cit., 90.

Cf. H. KONIG, op. cit., 311 ; A.S. DIAMOND, op. cit., 80.

Cf. A.S. DIAMOND, op. cit., 80.

Cf. H. KÖNIG, op. cit., 310 ; E.M. WEYER, op. cit., 225.

Cf. J. MALAURIE, op. cit., (Les derniers rois de Thule), 179.

Cf. G. STEFANI - G. LEVASSEUR, op. cit., (Droit Pénal...), 32.

Cf. H. KÖNIG, op. cit., 307-308 ; E.M. WEYER, op. cit., 226 ; A.S. DIAMOND, op. cit., 80 ; A. DORSINFANG-SMETS, op. cit., 31 ; K. BIRKET-SMITH, op. cit., (Mœurs et coutumes), 139.

9 Cf. H. LEVY-BRUHL, op. cit., (L'Ethnologie juridique...), 1169-1170.

10 Cf. J. MALAURIE, op. cit., (Les Civilisations Esquimaudes...).

11 Cf. E. WEYER, op. cit., 200. 
L'exécution qui suit l'accomplissement de tels actes revêt un caractère public qui la fait échapper au cercle des vendettas : c'est parce qu'avant d'agir le ou les exécuteurs ont obtenu l'accord de la communauté qu'ils n'encourent pas de représailles de la part des parents de leur victime. D'ailleurs, afin de couper court à toute éventualité de revanche familiale, la communauté désigne souvent comme exécuteurs les parents de la victime : en 1921, le leader des Arviligjuarmiut (Inuit Netsilik) dut ainsi exécuter son frère ; à Point-Barrow (Nord-Ouest Alaska), le frère et l'oncle tuent leur parent, qui avait lui-même tué une douzaine de personnes. Cependant, sauf sur la côte Ouest du Groënland, quand le shaman dénonce un individu coupable de sorcellerie, le fautif peut être tué par les parents de la victime de la sorcellerie.

Enfin, on notera que si l'exécution est la modalité la plus brutale d'intervention du groupe, celui-ci peut ainsi réagir par d'autres types d'action : abandon ou ostracisme du délinquant, qui ont souvent pour lui un effet synonyme. (D'ailleurs le mot employé pour désigner l'abandon - qivituk - signifie aussi "suicide"...)

Une série d'exemples peut illustrer ces principes généraux :

o Les plus nombreux concernent les Inuit Netsilik :

- À Pelly Bay, entre 1920 et 1922, sévissait une sévère famine. Un couple, accompagné de sa petite fille, rendit visite au père de l'épouse. Ce dernier, profitant d'une absence momentanée du couple, mangea sa petite-fille. Au retour, les parents comprirent ce qui s'était passé, mais ne manifestèrent aucune réaction. Trois semaines plus tard, alors qu'au cours d'une expédition de chasse un phoque venait d'être capturé, le grand-père s'avança pour prendre la part qui lui était coutumièrement due : à ce moment, les chasseurs le maîtrisent et, en silence, le dévêtent entièrement. Il mourut gelé au moment où il atteignait son iglou. Seule une confession publique devant le village rassemblé aurait pu le sauver ${ }^{1}$.

- Toujours à Pelly Bay, Uvdloriasugsuk, dont le frère était devenu [p. 73] un ennemi public, fut désigné comme plus proche parent par le village pour l'exécuter. Il dut aller trouver son frère, lui expliquer les raisons de la condamnation, et lui donna le choix du mode d'exécution. Puis il le tua ${ }^{2}$.

- En Terre du Roi Guillaume vivait un grand shaman, de surcroît très bon chasseur. Mais il prenait les femmes de ses compagnons, se faisait entretenir, et avait déjà tué sept personnes. La communauté décida son exécution, mais sans désigner officiellement de vengeur. Quelque temps après, un jeune homme tua le shaman ${ }^{3}$.

- Un individu s'était rendu coupable de sorcellerie : sous la pression de l'opinion publique, sa famille décida de l'exécuter. Il semble que les non-parents

Cf. J. MALAURIE, op. cit., (Les derniers rois de Thule), 179-180.

Cf. K. RASMUSSEN, op. cit., (Du Groënland au Pacifique), 232-233.

Cf. G. DE PONCINS, Kablouna (Paris, 1947), 170. 
ne prirent pas part à cette décision, mais il ne faut pas oublier que la délibération eut lieu sous une forte pression de la communauté, et que le cercle des parents peut être défini très extensivement : alliés, adoptés, amis, etc. Mais, en général, l'exécuteur est toujours le plus proche parent mâle de l'exécuté ${ }^{1}$, ce qui est bien le signe d'une décision de la communauté, au contraire d'une vendetta familiale. Ces données sont confirmées (pour les Netsilik) par d'autres observations similaires ${ }^{2}$.

D'une façon plus générale, G. Van den Steenhoven estime que, chez les Netsilik, il y a décision communautaire d'exécution du perturbateur lorsque trois catégories de règles sont enfreintes : partage collectif des plus importants moyens de subsistance ; obligation de participer à la chasse (sauf pour les infirmes); toute personne qui veut s'installer dans un camp doit préalablement obtenir le consentement de ceux qui s'y trouvent déjà ${ }^{3}$. (Seul le dernier cas paraît être une particularité Netsilik, non dans le contenu de la norme, mais dans le degré de gravité de la sanction qui frappe sa transgression.)

o Chez les Inuit du Cuivre : D. Pryde rapporte plusieurs cas ${ }^{4}$ :

- Aqsuq, chasseur de Bathurst Inlet, tuait les maris des femmes [p. 74] qu'il désirait, et mettait tout le village au défit de l'en empêcher. Pendant un certain temps, la communauté apeurée le laissa faire, puis décida son exécution. (D'une façon générale, avant que la décision ne soit prise, on laisse toujours le perturbateur aller jusqu'à la limite du tolérable). Alors que, dans la plupart des cas, l'exécuteur est le plus proche parent du condamné, celui d'Aqsuq fut un certain Uqsina, dont Aqsuq avait tué le père et le frère. Uqsina fut désigné par la communauté, mais ne brûlait pas d'envie de remplir sa mission. Il n'avait pas tort car une fois Aqsuq tué, les parents de ce dernier voulurent exercer la vendetta, ce qui, $a$ contrario, montre bien la sagesse du principe général d'exécution par le plus proche parent. D. Pryde rapporte, d'ailleurs, que dans les cas d'exécution décidée par la communauté qu'il a connus à Parry Island et Bathurst Inlet, l'exécuteur était, le plus souvent, le fils du condamné, ou son plus proche parent qui, de plus ou moins bon gré, accomplissait toujours sa mission. L'exécution, la plupart du temps, a lieu au cours d'une expédition de chasse (comme dans le cas d'Aqsuq), à l'aide d'un fusil, ou d'un couteau : dans ce dernier cas, il faut au moins deux exécuteurs, l'un soulevant les bras de la victime, l'autre lui enfonçant le couteau sous l'aisselle.

- D. Pryde rapporte également un cas de sorcellerie : une vieille femme avait l'habitude de souffler à la figure de ceux qu'elle rencontrait. Le groupe interpréta cette pratique comme un acte de sorcellerie et décida son exécution, qui fut assurée par son fils et un autre individu.

\footnotetext{
Cf. G. VAN DEN STEENHOVEN, op. cit., (Legal concepts), 51, 54-55.

Cf. A. BALIKCI, op. cit., (The Netsilik Eskimo), 192.

Ibid., 60-61.

Cf. D. PRYDE, op. cit., 62-69.
} 
o J. Malaurie rapporte qu'en Ungava (Nord de la province de Québec), un individu prenait systématiquement les femmes de ses congénères. Ces derniers l'obligèrent à construire son iglou à l'écart du camp, et allèrent l'y tuer ${ }^{1}$.

o Chez les Nunamiut (Alaska), l'individu qui s'est rendu coupable d'une faute grave et n'a pas voulu s'amender devant l'umialik et la communauté rassemblée dans le karigi ${ }^{2}$ peut encourir la peine de mort, décidée par le groupe ${ }^{3}$.

o [p. 75] Chez les Inuit Chugach, de façon quelque peu similaire, l'individu qui a subi un blâme public décerné par le chef du village devant la communauté et qui récidive peut se voir condamné à mort ${ }^{4}$.

o Chez les Inuit Centraux : Padlu, afin d'obtenir la femme qu'il désirait, avait tué son mari, et les parents qui avaient voulu le venger. Le leader des Akudmirmiut, informé de ces agissements, demanda alors à chaque membre du groupe si Padlu devait être tué. Ayant reçu l'accord de tous, il le tua dans le dos lors d'une expédition de chasse ${ }^{5}$.

Dans toute la série de cas que nous venons de citer ${ }^{6}$, l'action de la communauté est indéniable : il s'agit d'une décision concertée, nettement préalable à l'action qui n'a rien de spontané, s'exerçant suivant des formes codifiées. Ce mode d'intervention est évidemment très brutal : c'est l'ultime mesure de défense sociale, consistant dans la suppression du perturbateur. L'environnement ne laisse guère de choix : il est évident qu'il serait impossible chez les Inuit de mettre en œuvre une pratique similaire à ce qu'est chez nous l'incarcération. (Le seul "emprisonnement" pratiqué par les Inuit consiste dans des mesures psychologiques préventives qui, à divers degrés, isolent le coupable de la communauté).

Il existe cependant d'autres procédés d'intervention du groupe : plus souples, ils n'en sont pas moins directs et efficaces, et possèdent l'avantage d'économiser les vies humaines. II s'agit des duels judiciaires, dont on constate l'existence chez certaines populations, et qui sanctionnent certaines causes de conflits. C'est à leur étude que nous allons maintenant procéder, dans la dernière partie de ce chapitre.

\footnotetext{
Cf. J. MALAURIE, op. cit., (Les derniers rois de Thule), 178-179.

Cf. supra, p. 35.

Cf. W.H. OSWALT, op. cit., 178 ; L. POSPISIL, op. cit., (Law and societal structure), 423.

Cf. K. BIRKET-SMITH, op. cit., (The Chugach Eskimo...), 98.

Cf. F. BOAS, The Central Eskimos (Bureau of American Ethnology, Annual report, 6, 1888), 668.

6 L'exécution par décision de la communauté existe pratiquement chez toutes les populations Inuit, sauf sur la côte Est du Groënland (cf. E. ADAMSON-HOEBEL, op. cit.., 89). Peut-être cette exception est-elle due au faible degré de structuration politique de cette population et à sa faiblesse numérique?
} 
[p. 76]

\section{B) LES DUELS JUDICIAIRES ${ }^{1}$}

\section{$\underline{\text { Retour à la table des matières }}$}

Les duels judiciaires tels que les pratiquent les sociétés inuit constituent certainement un des terrains d'investigation les plus riches à la fois pour le juriste, le sociologue et l'ethno-psychiatre. En effet, nous allons voir qu'ils sont un des lieux de jonction entre le règlement des conflits privés et la préservation de l'intérêt public ; qu'ils existent surtout dans les sociétés inuit les plus pauvres en hommes et en ressources, ce qui montre qu'ils sont une réponse sociale à l'environnement ; et qu'enfin ils comportent un indéniable aspect psychothérapeutique, aussi bien pour les parties en cause que pour la communauté qui y assiste. Pour notre part, nous nous attacherons naturellement surtout à l'aspect socio-juridique de ces procédures, après avoir brièvement exposé leurs principes généraux.

\section{1- Principes généraux}

Le duel judiciaire est une des variétés de combat limité et réglé par la communauté qu'on rencontre dans les sociétés archaïques ${ }^{2}$. (On sait toute l'importance que revêtaient les ordalies dans le droit médiéval français ${ }^{3}$, et qu'on a pu parler d'ordalies dans le très ancien droit romain). La sanction qui y est mise en œuvre, tant au niveau psychologique que proprement judiciaire, joue une fois de plus sur la nécessité, pour l'individu, de se sentir relié au groupe, qui décide à la fin du combat qui est le perdant ${ }^{4}$. L'aspect psychologique est [p. 77] particulièrement sensible dans les duels judiciaires qui revêtent la forme de compétitions de chants. Ces chants satiriques ne font pas automatiquement référence aux motifs précis du conflit et comportent une large part d'éléments imaginaires, perçus comme tels par tous ${ }^{5}$ : ce ne sont donc pas des "preuves", mais plutôt des moyens psychothérapeutiques qui permettent aux individus de défouler leur agressivité, une sorte

1 Pour une étude générale, cf. surtout H. KÖNIG, op. cit., (Der Rechtsbruch...), 276-297. Cf. aussi, à titre accessoire : C.M. SMIDT - I.M. SMIDT, "Du chant au tambour aux cours de justice locales", dans Objets et Mondes XV - 2 (1975), 243-246 ; C.Y. CHARRON, "Le tambour magique : un instrument autrefois utile pour la quête d'un conjoint", dans Études/Inuit/Studies, vol. 2 no 1 (1978), 3-20 ; N. BEAUDRY, "Le katajjaq comme activité ludique de la société inuit", ibid., 5-53.

2 Cf. H. LEVY-BRUHL, "La preuve judiciaire chez les 'primitifs' ", dans Recueils de la Sté J. Bodin ; La Preuve, III (Bruxelles, 1969), 8 ; G. SAWER, op. cit., 42. On retrouve des compétitions de chant en Tasmanie : cf. H. KÖNIG, op. cit., 298.

Cf. A. DUMAS, Manuel d'Histoire du Droit français (Aix-en-Provence, s.d.), 49-51.

Cf. K. BIRKET-SMITH, op. cit, (Mœurs et coutumes...), 67-68.

Cf. infra, p. 82 sq. 
de psychodrame ${ }^{1}$. La communauté y trouve aussi son avantage : en plus de leur caractère judiciaire, ces compétitions sont un passe-temps social ${ }^{2}$, qui a lieu lors des longues soirées d'hiver, où l'ennui est pesant et l'environnement extérieur particulièrement dur.

Mais ces compétitions ont également une valeur socio-juridique : leur but principal est de permettre, par ce défoulement quasi-collectif, le rétablissement de la paix, par une autre voie que celle de nos systèmes judiciaires où la "justice" est établie d'après l'appréciation des responsabilités des parties en cause ${ }^{3}$. Modes de règlement pacifiques, ces duels ne peuvent évidemment sanctionner tous les types d'infractions ${ }^{4}$ : ils sont limités à celles d'un moindre degré de gravité, qui n'ont pas encore dégénérées en affrontements violents ${ }^{5}$.

Ces duels judiciaires obéissent à des formes codifiées ${ }^{6}$. Combat à coups de poing et de tête, lutte, compétition de chants. On trouve les combats à coups de poing chez les Inuit Centraux, le long du cercle polaire, de la baie d'Hudson au détroit de Behring ; ils sont inconnus chez les Inuit Polaires. La lutte est pratiquée en Sibérie, Alaska, Terre de Baffin, Nord-Ouest du Groënland. On trouve les compétitions de [p. 78] chants dans l'Est et l'Ouest du Groënland ${ }^{7}$, chez les Inuit Polaires, Iglulik, Caribou, dans l'Ouest alaskien et les îles Aléoutiennes, au Labrador $^{8}$. (Ces formes peuvent se mêler : ainsi, à Ammassalik (Côte Est Groënland), les chants s'accompagnent de coups de tête, alors que, sur la Côte Ouest, la compétition reste limitée à ces seuls chants ${ }^{9}$ ). La forme de ces compétitions est très précisément codifiée :

- Dans les combats de lutte, les deux combattants s'offrent successivement aux coups de leur adversaire sans lui résister, malgré la violence de ces attaques, portées en général au visage ; le "coup bas" est inconcevable ${ }^{10}$. Ces combats peuvent aussi revêtir la forme d'exercices d'endurance : une des parties tend son bras, pendant que son adversaire lui applique des coups répétés, toujours sur le même point, situé en haut du membre ${ }^{11}$. Dans le Nord-Est du Canada, les combattants

M. GLUCKMANN, op. cit., 306, les définit ainsi. "As in the joking partnerships analysed above, a standard pattern of abuse and derision is established between men, who indulge in it competitively for fun and prestige. It fits into the general derision used on all occasions".

Ibid., 305.

Cf. K. BIRKET-SMITH, op. cit, (Mœurs et coutumes), 178 ; E. ADAMSON-HOEBEL, op. cit., 99.

Cf. infra, p. 88.

Cf. G. SAWER, op. cit., 42 ; N.H.H. GRABURN, op. cit., (Eskimo Law...) 53.

Pour la description détaillée de ces formes, cf. H. KÖNIG, op. cit., 276-287 ; E. ADAMSONHOEBEL, op. cit., 92-99 ; E.M. WEYER, op. cit., 226-228.

7 Sur les compétitions de chants sur la côte ouest du Groenland, cf. Inge KLEIVAN, "Song Duels in West Greenland - Joking relationship and avoidance", dans Folk, 13 (1971), 9-36.

8 Pour ces localisations, cf. E. ADAMSON-HOEBEL, ibid., et la carte de H. KÖNIG, que nous reproduisons ci-contre.

9 Cf. H. KÖNIG, op. cit., 281-282, 287.

10 Cf. J. MALAURIE, op. cit., (Les derniers rois de Thule), 178.

11 Ibid. 
peuvent être armés d'un couteau ; s'il y a provocation, l'offensé se lève, poitrine en avant : ce n'est qu'après avoir été blessé qu'il ripostera ${ }^{1}$.

- Dans les compétitions de chants, les chants doivent obéir à des règles de composition et d'exposition très formalisées ${ }^{2}$.

Cependant, cette codification ne suffit pas à elle seule à en faire des procédures juridiques : toute règle de conduite sociale n'est pas juridique. Mais certains éléments, qu'il nous faut maintenant étudier, permettent de conclure à leur juridicité.

[p. 79]

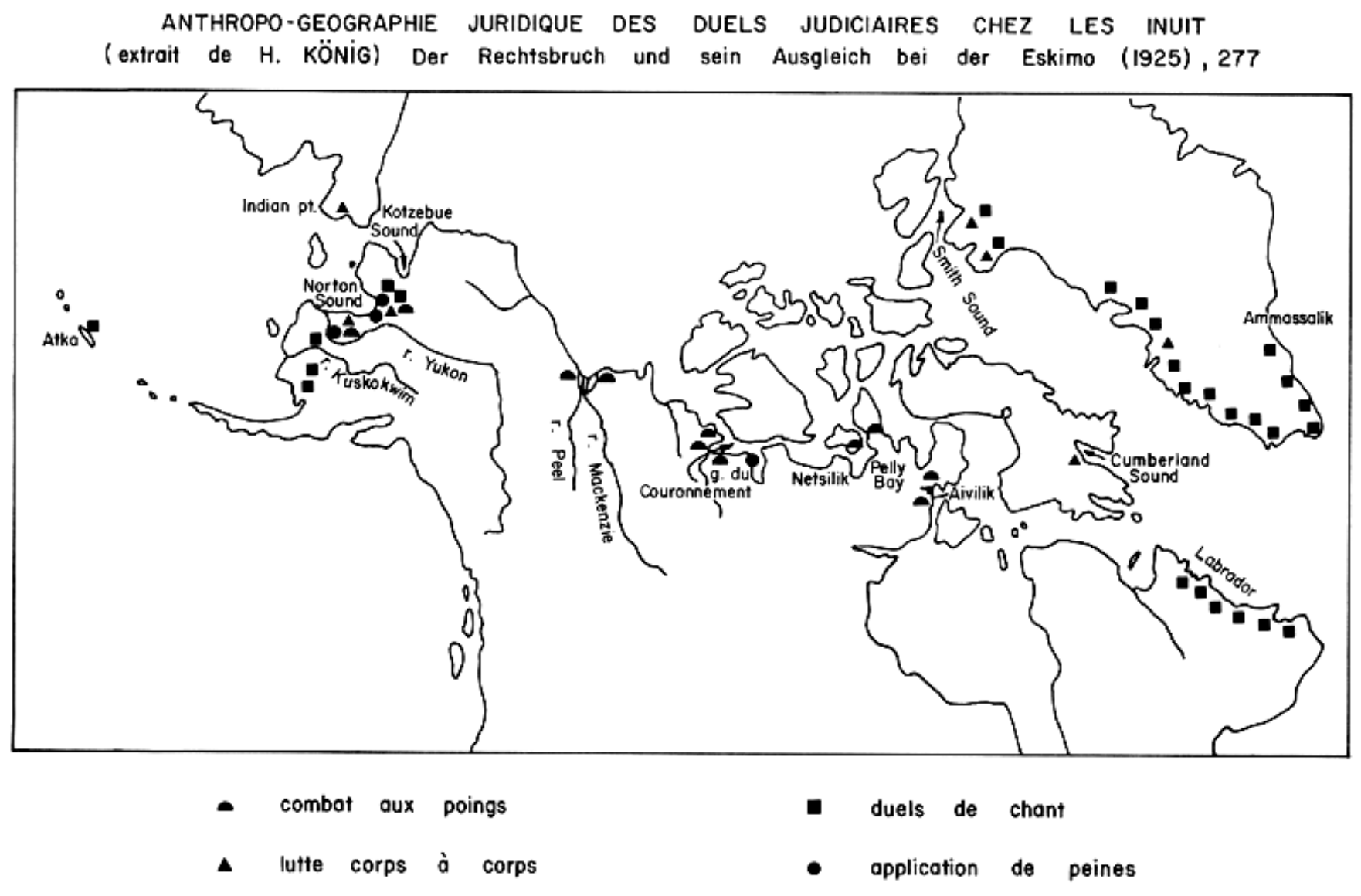

Ibid.

Cf. E. ADAMSON-HOEBEL, op. cit., 93 et supra p. 77 et n. 7. 
[p. 80]

\section{2- Juridicité des duels judiciaires chez les inuit : l'exemple fourni par les compétitions de chants ${ }^{1}$}

L'unanimité est loin d'être faite sur le caractère juridique et judiciaire de ces compétitions, ou beaucoup d'auteurs se refusent à voir autre chose qu'une forme non juridique de contrôle social ${ }^{2}$, ainsi qu'une sorte de diversion apportée à la monotonie de fa vie quotidienne. D'autres, en revanche, se prononcent pour leur juridicité ${ }^{3}$. La réponse n'est pas simple et ne saurait, à notre sens, être univoque : les compétitions de chants sont des procédés complexes, qui mêlent des éléments juridiques et non juridiques, judiciaires et non judiciaires : il n'y a qu'en les analysant de façon plus détaillée qu'on peut espérer parvenir à quelques données relativement claires. C'est ce à quoi nous allons consacrer les lignes qui suivent. On peut, afin d'arriver à mieux cerner ce phénomène, se poser trois questions : quel est le mécanisme par lequel le conflit est réglé dans ces compétitions, quel est le rôle de la communauté, quelle est la finalité de ces compétitions?

\section{a) Le mécanisme de la compétition de chants et le régime de la preuve}

Le problème qui se pose est celui de savoir quels éléments sont pris en considération pour déterminer qui sera gagnant, et perdant à l'issue de la procédure. Comme on le sait, dans nos systèmes judiciaires, l'acte juridictionnel consiste, à l'aide de divers moyens de preuve, à statuer sur les responsabilités des parties en cause à l'occasion [p. 81] d'un fait juridique précis, et prononcer en conséquence

1 Nous nous référerons surtout ici aux compétitions de chants pratiquées sur la côte Ouest du Groënland.

2 Ainsi M. GLUCKMANN, op. cit., 190 ; G. VAN DEN STEENHOVEN, op. cit., (Legal concepts...), 63.

3 Ainsi H. KÖNIG, op. cit., 281, 288-289. Mais KÖNIG y voit plus une forme de vengeance qu'une procédure judiciaire devant un "tribunal" : "Der Singstreit der Eskimo ist also nicht als prozessuales Mittel zur Feststellung des Rechtes oder Unrechtes, auf die erst die Sühne für den Rechtsbruch festgesetzt wird, anzusehen, sondern er ist eine durch das Herkommen geregelte Art der Rache und trägt als solche den Ausgleich schon in sich", (H. KÖNIG, op. cit., 290). Cf. aussi E. ADAMSON-HOEBEL, op. cit, 98 : "The song duels are juridical instruments insofar as they do serve to settle disputes and restore normal relations between estranged members of the community". A. DORSINFANG-SMETS, op. cit., 19 : "Il y a action légale puisque le redressement des torts, ou la sanction du coupable, est décidée et mesurée par une autorité qu'admet la société et que les mesures à prendre sont proposées selon des règles qui rencontrent l'adhésion du corps social", (cf. aussi, ibid., pp. 30-31). 
une sanction. Or, dans les compétitions de chants ${ }^{1}$, il y a un apparent manque de sanction. Le vaincu est, en effet, celui qui est à court de chants, ou dont les chants ont été jugés d'une qualité inférieure à ceux de son adversaire : après quoi le conflit est censé être réglé ${ }^{2}$ et ont souvent lieu un échange de cadeaux et une fête ${ }^{3}$. En réalité, la sanction est effective, mais doit être rangée dans la catégorie des sanctions psychologiques : étant donné le rôle de la communauté, qui est toujours l'autorité judiciaire et décide de l'issue du duel ${ }^{4}$, la sanction consiste dans la préférence qui a été donnée par le groupe aux chants de l'adversaire, et dans l'humiliation qui en résulte pour le perdant.

D'autre part, comme le note, à juste titre, H. König ${ }^{5}$ ces compétitions n'ont pas pour objet de déterminer quelle partie est dans son droit, mais laquelle est la plus habile à manier et composer les chants. Si bien que le vainqueur peut être celui qui, en regard des faits qui avaient nécessité le recours au duel judiciaire, avait transgressé une règle ... Nous saisissons bien là qu'il s'agit d'une procédure de règlement des conflits par le détour : les faits du litige ne sont pas directement examinés, et le conflit est vidé par le recours à un combat externe au litige luimême. On pense évidemment à une procédure de type ordalique, où les qualités montrées par le vainqueur dans un certain type de combat sont censées être un signe divin de son bon droit : dans [p. 82] l'impossibilité de preuves rationnelles, on suscite l'intervention du surnaturel ${ }^{6}$. Les auteurs se prononcent, en général, contre l'attribution d'un caractère ordalique à ces compétitions de chants ${ }^{7}$, au motif qu'elles ne mettent nullement en jeu le recours à des puissances surnaturelles. L'observation est juste, mais doit peut-être recevoir quelque nuance : les compétitions de chants ont au moins en commun avec les ordalies le fait qu'elles délaissent les moyens de preuve rationnels, et constituent un type de procédure par le détour, par la mise en jeu d'éléments extérieurs au litige ${ }^{8}$. C'est donc dire que,

1 Cf. 1. KLEIVAN, op. cit., 24 (Côte Ouest Groënland) ; G. VAN DEN STEENHOVEN, op. cit., (Legal concepts...), 30, 34, (Netsilik).

2 Pour G. VAN DEN STEENHOVEN, op. cit., 30, la réconciliation, chez les Netsilik, est loin d'être automatique. (Dans le même sens, cf. M. GLUCKMANN, op. cit., 305).

Cf. M. GLUCKMANN, op. cit., 190.

Cf. infra, p. 87 sq.

Cf. H. KÖNIG, op. cit., 281, 287.

Au Haut Moyen-Âge, les ordalies pouvaient être unilatérales, c'est-à-dire consister dans des blessures infligées à un justiciable (ordalies du fer rouge, de l'eau bouillante...) ou contradictoires : combat armé entre les deux plaideurs, ou leurs champions (preuve par bataille).Dans tous les cas, le vainqueur, ou celui dont les blessures s'étaient normalement consolidées - pour les ordalies unilatérales - était celui qui était dans son bon droit, puisque visiblement protégé par Dieu. Le sacramentum du très ancien droit romain résout aussi les litiges par le détour de l'interprétation augurale de la volonté divine (cf. supra p. 13).

7 "Von einem Vertrauen auf die Hilfe höherer Mächte, die dem Rechte zum Siege verhelfen werden, kann meines Erachtens gar keine Rede sein". (H. KÖNIG, op. cit., 287-288). Dans le même sens, cf. E. ADAMSON-HOEBEL, op. cit., 99.

8 Certains auteurs mettent, d'ailleurs, l'accent sur le caractère ordalique des duels judiciaires en général (cf. H. LEVY-BRUHL, op. cit., (La preuve judiciaire ...), 8 et chez les Inuit (cf. A. DORSINFANG-SMETS, op. cit., 30-31). 
dans ces types de débats, la preuve judiciaire n'est pas du tout mise au même rang que dans nos sociétés : la force probante est plus attachée à l'appréciation globale portée par la communauté sur l'ensemble de la personnalité d'un individu, appréciée dans une forme particulière : la compétition de chants ${ }^{1}$.

Ce caractère est d'autant plus affirmé si l'on remarque que, la plupart du temps, les chants n'évoquent pas les faite du litige, ou n'en traitent que très superficiellement ${ }^{2}$. Il en est notamment ainsi chez [p. 83] les Inuit Netsilik ${ }^{3}$, et ceux de la Côte Ouest du Groënland ${ }^{4}$ ainsi que ceux de la côte Est ${ }^{5}$. Cependant, cette règle, pour être générale, n'est pas absolue : certains chants montrent que les faits du litige sont cités.

L'analyse de quelques textes peut, à titre d'exemple, illustrer le principe ... et ses exceptions.

o Le premier chant (Est Groënland) montre comment les parties, après avoir pris comme point de départ les faits générateurs du conflit s'en éloignent de plus en plus, et mettent en jeu d'autres aspects de leur personnalité ${ }^{6}$ :

( $\mathrm{E}$ a pris la femme du vieux $\mathrm{K}$ qui l'avait quittée. $\mathrm{K}$ veut la reprendre mais $\mathrm{E}$ s'y oppose, d'où le recours à une compétition de chants).

$\mathrm{K}$ : "Maintenant je vais attaquer avec des mots, de des petits mots aiguisés, comme les éclats bois que je fends avec ma hache. Un chant de l'ancien temps, un souffle des ancêtres. Un chant destiné à ma femme. Un impudent, un sombre idiot me l'a volée, a essayé de la di-

1 Cette conception particulière du débat judiciaire est propre à beaucoup de populations archaïques : cf. H. LEVY-BRUHL, op. cit. (La preuve judiciaire...), 7 ; "Il peut à peine être parlé de preuve proprement dite, mais chacun utilise les moyens les plus propres à capter la faveur du groupe" (Ibid.). Dans le même sens, cf. M. GLUCKMANN, op.cit., 304, et A. BALIKCI, op. cit., (The Netsilik Eskimo), 189.

2 "Let it be remembered that "right" is immaterial to the singing or its outcome (...). As the court-room joust may become a sporting-game between spanning attorneys-at-law, so the juridical song contest is above all things a contest in which pleasurable delight is richly served, so richly that the dispute-settlement function is nearly forgotten. And in the forgetting, the original end is the better served" (E. ADAMSON-HOEBEL, op. cit., 99). Dans le même sens, cf. N.H.H. GRABURN, op. cit., (Eskimo law...), 53.

3 Cf. G. VAN DEN STEENHOVEN, op. cit., (Legal concepts...), 30, 31 ; M. GLUCKMANN, op. cit., 304-305 : Steenhoven ne cite qu'un seul cas où il y ait eu référence au fait précis générateur du litige (un Inuk avait réduit son neveu à la famine en utilisant sans réelle nécessité ses provisions de viande pour nourrir ses chiens). Cette observation est confirmée par A. BALIKCI, op. cit. (The Netsilik Eskimo), 189, qui insiste à juste titre, sur la valeur cathartique de cette procédure.

4 "It does not emerge from the primary sources of the 18th Century that there was any endeavour to let justice be done. It has rather been a case of the man getting the most applause who could advance some good points in a convincing way" (I. KLEIVAN, op. cit., 25).

Cf. le texte cité infra.

6 Cité par E. AJDAMSON-HOEBEL, op. cit., 94-96. 
minuer. Un scélérat qui aime la chair humaine, un cannibale des jours de famine.

$\mathrm{E}:$ C'est une insolence qui soulève le cœur, une arrogance ridicule et de l'effronterie. Quel [p. 84] chant satirique ! Dire qu'il est censé montrer ma faute. Tu voudrais mettre la crainte dans mon cœur. Je ne me soucie pas de la mort. Eh ! Tu chantes à propos de ma femme qui était ton amie. Tu ne l'aimais pas tant que cela, car elle était très seule. Tu as oublié de lui donner de la valeur dans le chant, dans les intrépides duels de chants. Maintenant elle est à moi. Et jamais plus elle n'ira chez des amoureux illusoires, qui chantent. Séducteur de femmes dans d'étranges maisons".

Dans cette première phase, les deux parties se tiennent strictement au fait du litige : le rapt de la femme. Il est intéressant de noter que E donne au chant une valeur judiciaire, puisqu'il cherche à ridiculiser le chant de K qui "est censé établir sa faute". Cependant, il ne s'agit pas d'un exposé froid et objectif des faits : l'injure et le ridicule jouent leur rôle, et d'autres faits commencent à apparaître : K accuse E d'être un anthropophage, comportement qu'acceptent difficilement les Inuit, d'une façon générale.

Dans la seconde phase des chants, le rapt de la femme n'est pratiquement plus incriminé : chacun des deux adversaires emploie pour attaquer l'autre une méthode différente. $\mathrm{K}$ - celui qui s'est vu privé de sa femme - accuse $\mathrm{E}$ de tentative de meurtre. Il s'agit sans doute par là de montrer que le rapt de la femme s'inscrit dans la logique d'un comportement : E est un individu dangereux, prêt à n'importe quel forfait :

$\mathrm{K}$ : "Laisse-moi aussi suivre l'umiak en kayakeur ! Suivre le bateau avec les chanteurs. Comme si j'étais effrayé, comme si j'étais à genoux, lorsque je poursuis le pagayeur. Ce n'est pas étonnant qu'il soit si content : c'est lui qui a presque tué son cousin, c'est lui qui a failli harponner son cousin. Ce n'est pas étonnant qu'il soit si satisfait, qu'il montre tant de joie".

$\mathrm{E}$ rétorque non en répondant sur ce fait précis que $\mathrm{K}$ lui reproche, mais en lui renvoyant la balle, exposant quel est selon lui le motif de son attitude calomniatrice :

E- "Mais je trouve cela risible. Je suis content, car c'est toi, K. qui est un meurtrier ; c'est toi qui es jaloux de tout cela, qui excite ton envie. Car tu n'as pas plus de trois femmes, et c'est trop peu à ton goût, c'est pourquoi tu es jaloux. Tu devrais les donner [p. 85] en mariage à d'autres hommes. Ainsi tu pourrais avoir ce que leurs maris leur donneraient". 
$\mathrm{E}$ répond donc par la dérision. Mais $\mathrm{K}$ reprend sur le thème qu’il a choisi : "Ceci ne te regarde pas (...) tu as entrepris de tuer tes compagnons".

On voit donc que si le conflit particulier n'est jamais oublié, les éléments mis en jeu lui sont de plus en plus extérieurs. Il faut cependant bien noter qu'il ne s'agit pas là de digressions gratuites : par l'appel à des faits extérieurs, ou des remarques sur la psychologie de son adversaire, chacun cherche à montrer qu'il s'agit d'un mauvais individu, ce qui explique donc qu'il soit le fautif dans la cause du conflit. On voit donc que le lien avec l'objet précis du débat est loin d'être perdu. Au moins, dans ce chant, la compétition a bien un caractère judiciaire : le régime particulier de la preuve ne doit pas nous tromper sur ce point. I. Kleivan fait d'ailleurs observer que, sur la Côte Ouest du Groënland, la mise en jeu de griefs purement imaginaires est considérée comme une infraction aux règles du duel ${ }^{1}$. (Il reste vrai que, dans ce cadre, on utilisera tous les moyens pour ridiculiser l'adversaire, ainsi que l'auto-satisfaction. ${ }^{2}$ )

Mais on trouve sans peine des exemples de chants qui sont en relation directe avec l'objet du conflit ${ }^{3}$ :

o Côte Ouest du Groënland :

- A "Tu m'as volé un biscuit l'autre jour, pendant que le marchand répartissait les provisions".

- B "Tu as raison, j'ai volé un biscuit l'autre jour, mais j'avais faim. Toi, tu as volé la seule ligne de pêche d'Illamut, qui lui servait à nourrir [p. 86] toute sa famille. Qui a le plus mal agi, de toi ou de moi ?" ${ }^{4}$.

A restant coi, l'assistance donna raison à $\mathrm{B}$. Outre le fait que le chant et la cause du conflit sont directement liés, il est intéressant de noter que l'acte judiciaire accompli par la communauté est au moins autant le jugement d'une compétition que celui de l'élément matériel du délit : B obtient les suffrages de l'assistance peut-être parce que la faim a été jugée comme une excuse absolutoire de son larcin, mais surtout parce qu'il a su mettre son accusateur en difficulté.

1 "...it was considered a breach of ethics to manipulate the facts and assert things for which there was no foundation in truth, but whether or not the statements made were in keeping with the facts, the contests have been an occasion for the demonstration of the prevailing moral code" (I. KLEIVAN, op. cit., 11).

2 "It is apparent from the examples of satirical songs that you not only ridiculed your opponent for his deficiencies, but could also belittle him by drawing attention to yourself at his expense" (Ibid., 21).

3 G. VAN DEN STEENHOVEN (op. cit., 32) reconnaît que chez les Netsilik certains chants font, en effet, référence à l'objet précis du conflit, mais avoue ne pas parvenir à donner une explication satisfaisante de ce phénomène.

4 Cité par I. KLEIVAN, op. cit., 11. 
o Un autre chant consiste tout entier dans une menace (Ouest Groenland) : A a induit B, la femme de $\mathrm{C}$, a saboter le kayak de son mari afin qu'il (C) se tue et qu'elle puisse l'épouser (A). Mais l'attentat échoue et $C$ s'adresse à $A$ et $B$ en ces termes ${ }^{1}$ (on notera l'ironie que $\mathrm{C}$ emploie envers lui-même) :

0

\begin{abstract}
"Ah, combien je me sens plein de doutes à ce sujet ! Combien mon faible esprit me rend incertain dans mon chant ! Cependant il pourrait m'arriver de faire un chant d'accusation contre lui. Comme il est stupide que maintenant j'ai vraiment à faire du bruit à son sujet. Lorsque nous étions vers le Nord, à Kialineq, il arrivait fréquemment qu'elle me mette en colère si bien que j'avais l'habitude de la battre. Je n'avais pas tort d'être fâché. J'étais comme d'habitude mécontent de son travail, car le dessus de mon kayak était déchiré. Il vint à s'ouvrir (...) Combien j'ai été stupide de ne pas le traiter de la même façon, j'aurais dû lui donner un coup de couteau. Quel malheur que je sois si indulgent avec toi, quel malheur que je te marque de la considération. Tu es une canaille sans cervelle qui mérite ma colère".
\end{abstract}

o D'après E. Kent Kane ${ }^{2}-$ mais $H$. König reste très réservé et croit plutôt à une erreur d'interprétation de sa part ${ }^{3}$ - à Upernavik (Ouest Groenland) la compétition de chants fonctionne comme un véritable tribunal : les chants terminés, les angakok se concertent et prononcent une peine à l'encontre de l'accusé, ou de l'accusateur, s'il n'a pu prouver la véracité de ses dires.

[p. 87]

Pour conclure sur ce point, on voit donc, d'après les exemples (évidemment non exhaustifs) que nous avons cités, que si les griefs précis motivant le conflit ne sont peut-être pas, en général, évoqués, les chants ne perdent jamais tout lien avec eux. Ils ont seulement pour but de faire porter une appréciation globale sur le comportement des parties, mais tout en montrant l'habileté de chacune dans cette forme de compétition, habileté dont dépend évidemment l'issue du conflit : c'est là l'élément qui inclinerait le plus le juriste à la réticence. Mais croit-on que dans nos systèmes judiciaires, le mécanisme des plaidoiries ne participe pas au moins en partie du même esprit ? Pour déterminer la responsabilité du délinquant, son défenseur cherchera à expliquer son acte, mais par la prise en compte d'éléments très antérieurs : son éducation, milieu familial, traits de caractère, etc. D'autre part, le succès d'une plaidoirie dépend, non seulement du bon droit dans lequel se trouve éventuellement le plaideur, mais également de l'intelligence et du talent oratoire

Cité par E. ADAMSON-HOEBEL, op. cit., 96-97.

Cf. E.K. KANE, Arctic Explorations (Philadelphia and London, 1856).

Cf. H. KÖNIG, op. cit., 290. 
de son avocat. On voit donc que, malgré les apparences, les compétitions de chants peuvent revêtir une forme processuelle.

Leur caractère juridique et judiciaire nous est confirmé par d'autres éléments, qu'il nous faut maintenant examiner.

\section{b) Caractère "public" des compétitions de chants : le rôle de la communauté}

La communauté intervient à deux niveaux :

- Indirectement, par la coutume, qui est son expression, et régit les compétitions de chants, qui sont des formes de règlement des conflits codifiées ;

- directement, en se manifestant au cours de la compétition.

Situons-nous successivement à chacun de ces deux niveaux :

\section{- La codification coutumière}

Rien de moins anarchique, de plus réglé qu'une compétition de chants : la coutume, expression du groupe, s'impose aux parties et les oblige à vider leur conflit en des formes pré-établies ${ }^{1}$. Le rite joue d'ailleurs un rôle précis : non seulement il sacralise, et donc frappe les participants ${ }^{2}$, mais [p. 88] il joue aussi le rôle de mémoire. (C'est une des raisons pour lesquelles le droit des époques archaïques très ancien droit romain et Haut Moyen-Âge - est très formaliste). Les combattants ne doivent user que de moyens définis par avance ; la communauté, toujours présente, est réunie dans des cadres différents : dans le karigi en Alaska, en plein air au Groënland ${ }^{3}$. Hans Egede, au XVIII ${ }^{\mathrm{e}}$ siècle, donne les principales règles concernant les Inuit de la côte Ouest du Groënland : celui qui se croit victime d'un préjudice défie son adversaire à un duel devant la communauté ; chaque partie prépare des chants en vue de cette assemblée. Le jour venu, chacun est assis : le demandeur se lève et commence à chanter en s'accompagnant d'un tambour, le défendeur se tient près de lui et n'intervient pas avant la fin du chant. Puis, il chante à son tour. La scène se reproduit autant de fois que nécessaire, et le perdant est celui qui se trouve à court de chants ${ }^{4}$. Il est de la plus haute importance que, quel que soit le contenu du chant qui l'attaque, la partie silencieuse ne manifeste aucune réaction, et ne chante qu'à son tour ${ }^{5}$. D'après $\mathrm{H}$. Egede, il semblerait donc que l'assistance n'exerce pas de rôle judiciaire : le vaincu est celui qui ne trouve

\footnotetext{
Cf. supra, p. 77 et n. 7.

Cf. A. DORSINFANG-SMETS, op. cit., 35.

Cf. H. KÖNIG, op. cit., 288-289.

Cf. I. KLEIVAN, op. cit., 10.

Ibid., 17.
} 
plus rien à dire ${ }^{1}$, soit par manque d'imagination, soit parce que les thèmes développés par son adversaire sont trop accablants pour lui ${ }^{2}$.

Nous allons voir en fait que la communauté intervient très activement dans ces duels ${ }^{3}$. Mais, auparavant, citons encore un point important à propos de la règlementation coutumière de ces compétitions : la matière des cas qu'elles peuvent régler. En effet, tout différend n'est pas susceptible de donner lieu à un duel judiciaire. Le pugilat et les coups de tête peuvent sanctionner toutes les querelles, sauf les homicides, ainsi que les compétitions de chants ; la lutte peut sanctionner les crimes de sang ${ }^{4}$. Cependant, sur le Côte Est du Groënland, pauvre en hommes et en moyens de subsistance, la compétition de chants peut, contrairement à la règle générale, se substi-[p. 89] tuer à la revanche sanglante en cas de meurtre : celui qui est trop faible pour se venger, ou particulièrement bon chanteur, peut la déclencher ${ }^{5}$.

\section{- Rôle de la communauté au cours de la compétition}

C'est ce rôle qui donne à la compétition de chants son caractère d'action publique exercée par le groupe, et la distingue d'une forme particulière, pacifique, de vengeance privée. Plusieurs observations mettent en relief l'intervention de la communauté.

- Nous venons de voir ${ }^{6}$ que, sur la Côte Ouest du Groënland, le premier acte du demandeur à suivre le défi qu'il adresse au défendeur est de le convoquer à une assemblée ultérieure de la communauté. D'ailleurs, chaque partie, avant la date de la réunion, prépare ses chants.

- Chez les Netsilik, les chants peuvent être improvisés, ou préparés longtemps à l'avance : mais dans les deux cas, ils se déroulent devant la communauté, et dans le kadgek, la grande maison d'hiver ${ }^{7}$. Comme sur la Côte Ouest du Groënland, chaque chanteur doit attendre son tour ${ }^{8}$. Les familles des participants les aident à composer leurs chants ${ }^{9}$, et les femmes forment d'ailleurs un chœur qui accompagne chaque partie ${ }^{10}$. Il est important de noter, du point de vue processuel, que c'est au cours de la préparation des chants que l'enquête se fait: les parents de chaque partie lui disent tout ce qu'ils savent sur son adversaire afin qu'elle inclue ces faits dans son chant et remporte ainsi plus facilement la victoire ${ }^{11}$. La compé-

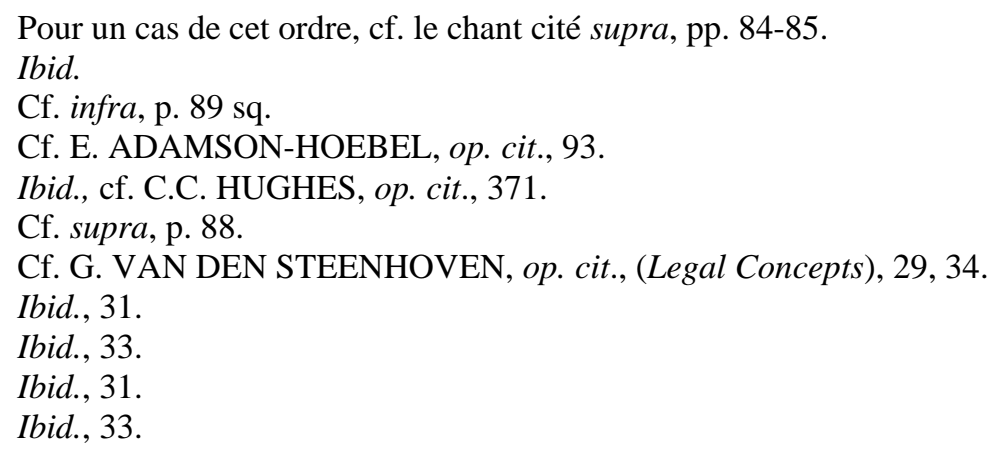


tition se termine de la même façon que sur la Côte Ouest du Groënland : celui qui se trouve à court de chants a perdu ${ }^{1}$. On pourrait donc croire que tout dépend de la seule habileté de chaque compétiteur, et que la communauté assemblée ne joue au mieux qu'un rôle de témoin. En fait, il n'en est rien : tout au long du duel elle se manifeste, et influe ainsi sur le comportement, [p. 90] le "moral" de chaque partie. G. Van den Steenhoven écrit ainsi, à propos des Netsilik :

"The intensely interested audience acts as arbitrator by giving its applause, and gradually withholding it from the loser" ${ }^{2}$.

K. Rasmussen fait lui aussi observer que :

"...if one of the opponents starts showing more and more that he feels ashamed (through the applauding accusations of the other), his contentedness decreases, and so does the number of his supporters" ${ }^{3}$.

Sur la Côte Ouest du Groënland, à Upernavik, E. Kent Kane rapporte des faits similaires :

The accused meanwhile is silent ; but, as the orator pauses after a signal hit or to flourish a cadence on his musical instrument, the whole audience, friends, neutrals, and opponents, signalize their approval by outcries as harmonious as those which we sometimes hear in our town-meetings at home. Stimulated by the applause, and warming with his own fires, the accuser renews the attack..." ${ }^{4}$.

I. Kleivan note (pour la Côte Ouest du Groënland) que cette participation de l'assistance peut être un facteur d'inégalité entre les parties, celui qui dispose de partisans et parents les plus nombreux dans l'auditoire étant évidemment avantagé ${ }^{5}$, ce qui peut même tourner à l'affrontement entre des communautés différentes, par l'intermédiaire de leurs membres parties au duel ${ }^{6}$.

Cf. G. VAN DEN STEENHOVEN, op. cit., 31.

Ibid. Dans le même sens : ibid., 33-34.

Cité par G. VAN DEN STEENHOVEN, op. cit., 33.

E.K. KANE, op. cit., 11, 128-129 (cité par E.M. WEYER, op. cit., 226). Cf. aussi E. ADAMSON-HOEBEL, op. cit., 96.

Cf. I. KLEIVAN, op. cit., 25.

Ibid., 14. 
On objectera que, si ces diverses observations prouvent bien l'intervention de la communauté, elles n'établissent pas son rôle judiciaire ou arbitral : en effet : l'évocation des faits objets du litige n'est pas obligatoire ; la communauté n'est pas seulement juge, [p. 91] mais partie, puisque dans une certaine mesure des "clans" soutiennent leurs champions ; il s'agit surtout d'un passe-temps plus que d'un jugement ${ }^{1}$; et leur efficacité n'est pas certaine ${ }^{2}$.

Il est vrai que, si on cherche à retrouver dans ces compétitions de chants les mêmes aspects que revêtent les sessions de nos institutions judiciaires, on sera immanquablement conduit à dénoncer leur non-juridicité. Mais, à notre sens, il y a bien pourtant là phénomène juridique et judiciaire, sous une forme particulière à une société archaïque. En effet :

- La justice se présente ici sous une forme plus passionnelle, trait commun à de nombreuses autres sociétés archaïques: "Il peut à peine être parlé de preuve proprement dite, mais chacun utilise les moyens les plus propres à capter la faveur du groupe..." ${ }^{3}$. Les "garanties" dont jouit le plaideur peuvent donc paraître peu étendues en regard de la souplesse de la procédure arbitrale ${ }^{4}$. Mais on doit bien comprendre ${ }^{5}$ - et répéter - que l'essentiel réside dans l'autorité du groupe qui, s'exprimant dans son approbation ou sa désapprobation, est censée mettre un point final au conflit, plus que dans la satisfaction donnée aux prétentions d'une des parties à la suite d'une analyse rationnelle des faits : encore une fois, l'individu doit s'effacer devant le groupe. En ce sens, l'acte arbitral peut aussi être arbitraire : le principal est qu'il mette fin au conflit. Il est donc une manifestation particulièrement puissante de souveraineté, un acte public par excellence.

[p. 92]

- Si l'évocation des faits du litige n'est pas obligatoire, c'est parce que, souvent, elle n'est pas nécessaire : en effet, dans des petites communautés, tout le monde est au courant de la vie de chacun. D'autre part, le régime de la preuve est différent de celui de nos systèmes juridiques : il passe beaucoup moins par l'appréciation de ces faits déterminés que par la prise en considération du total de la personnalité des parties en litige ${ }^{6}$. C'est la raison pour laquelle les chants contiennent des allusions à des actions des parties extérieures au litige. Le procé-

1 G. VAN DEN STEENHOVEN, op. cit., 32, rapporte "My informants all used expressions like 'great fun', or 'quite amusing' when talking about these kadgek songs".

2 G. VAN DEN STEENHOVEN, ibid., 36, doute que toutes les compétitions de chants mettent réellement fin à l'état conflictuel entre les parties.

Cf. H. LEVY-BRUHL, op. cit. (La preuve judiciaire), 7.

"...les procès (dans les sociétés archaïques) se déroulent aussi suivant des règles plus souples ce qui a, du reste, pour contrepartie, l'inconvénient de donner moins de garanties aux justiciables. Cela est presque évident dans les sociétés les moins évoluées, où il n'existe pas de tribunaux organisés. Les contestations s'y présentent souvent sous forme d'arbitrages, sous cette réserve que, par ce terme, on entende, non pas un acte privé, mais un véritable jugement rendu au nom du groupe social". (H. LEVY-BRUHL, op. cit. (L'Ethnologie juridique), 1162.

Cf. infra, p. 92.

Cf. supra, p. 87. 
dé n'a en lui-même rien d'irrationnel et, contrairement à ce que pensent beaucoup d'auteurs, n'ôte pas à ces compétitions leur caractère judiciaire : il est normal d'apprécier un fait à la lumière d'un comportement global. Le fait étant bien connu de tous, c'est ce comportement global qu'il faut expliciter : un élément d'appréciation est constitué par l'habileté que nous montre chacune des parties à révéler les travers de son adversaire dans la forme codifiée du chant. Quand la communauté se prononce sur cette aptitude, elle exerce donc bien un rôle judiciaire, dépassant le simple divertissement social. (Et, encore une fois, croit-on que chez nous le jugement rendu par la Cour ne dépend que du bon droit des parties, et non aussi du talent de leurs orateurs?).

- Si chaque partie compte des supporters dans l'assistance, il n'en reste pas moins vrai, d'après la quasi-totalité des observations, que l'autorité arbitrale ne consiste pas dans la victoire d'un "clan" sur un autre : c'est la communauté tout entière qui s'exprime dans la solution finale donnée au conflit. D'ailleurs, s'il en était autrement, le conflit ne pourrait se régler ainsi, les concepts arithmétiques (majorité relative, absolue, etc. ...) de la démocratie n'ayant pas de sens chez les Inuit. Ce qui ne signifie évidemment pas que le conflit soit automatiquement réglé : il se peut que, dans certains cas, cette procédure de règlement échoue. Mais, encore une fois, ce n'est pas parce qu'un mode de règlement des conflits s'avère parfois inopérant qu'il perd, pour autant, son caractère normatif, institutionnel, de procédure, que le groupe social, par le biais de la coutume, adopte et sanctionne : le phénomène juridique - et judiciaire - se définit plus par son intention répétitive que par une absolue régularité d'application, que ne possèdent même pas obligatoirement les faits purement [p. 93] physiques. Tout ce qui est humain comporte une part d'aléatoire, et le droit n'échappe pas à la règle.

- D'autre part, il est vrai que ces compétitions de chants sont aussi - mais pas seulement - des phénomènes sociaux de divertissement. Ce qui ne suffit nullement à leur enlever leur caractère judiciaire. La faveur que montre le public, dans nos sociétés, aux grands procès criminels - dont l'objet est pourtant en général tragique - ne témoigne-t-elle, pas qu'ils ont aussi valeur de divertissement ? ${ }^{1}$ Le psychiatre dira mieux que nous les raisons profondes de cet intérêt : il nous suffit de constater que divertissement et judiciarité ne sont nullement exclusifs l'un de l'autre. Ceci est d'autant plus vrai que, chez les Inuit, les compétitions de chants font très fréquemment appel à la moquerie, la dérision ${ }^{2}$, procédés propres à provoquer l'amusement de l'assistance. Enfin, n'oublions pas que, dans ces mêmes sociétés, tout doit être au maximum sublimé dans le sourire ...

- Enfin, la finalité de ces compétitions réside, avant tout, dans le rétablissement de la paix sociale : c'est ce que nous allons étudier dans les lignes qui suivent.

Cf. supra, p. 91 n. 1.

Cf. G. VAN DEN STEENHOVEN, op. cit., 29 ; E. ADAMSON-HOEBEL, op. cit., 96 ; I. KLEIVAN, op. cit., 17. 


\section{c) La finalité des compétitions de chants}

"...les observateurs paraissent d'accord pour déclarer que les procès ordinaires n'ont pas pour but principal d'appliquer une sanction, mais de réparer les déséquilibres sociaux qui peuvent se produire à cette occasion, car tout procès suppose qu'une règle coutumière est violée ou contestée (...) sans doute notre système judiciaire doit-il apparaître à beaucoup de peuples primitifs comme trop rigoureux. Cela s'explique fort bien, à mon sens, par leur souci très puissant d'éviter à tout prix une rupture ou une diminution de la cohésion sociale, tandis que cette préoccupation étant moins vive dans les États modernes, plus fortement structurés, ils peuvent exiger de leurs membres une plus stricte obéissance aux règles qu'ils prescrivent" ${ }^{1}$.

[p. 94]

Écrites à propos des sociétés archaïques en général, ces lignes expriment fort bien la finalité de la "justice" chez les Inuit, telle que nous la révèlent les compétitions de chants : à la différence de la vendetta familiale, elles constituent un mode public de règlement pacifique des conflits. Leur but n'est donc pas de permettre à une des parties d'annihiler, de déterminer strictement les responsabilités, mais de rétablir la paix sociale par l'accomplissement de deux objectifs corollaires :

- Permettre aux parties de défouler leur agressivité dans leurs chants : c'est l'aspect psycho-thérapeutique de cette procédure qui, comme le note fort justement Hoebel ${ }^{2}$, constitue un avantage par rapport à bien d'autres systèmes judiciaires. (On sait la valeur qu'attachent les traitements psychiatriques aux psychodrames pour la résolution des conflits psychologiques.)

- Mais aussi apprécier la valeur globale du comportement de chaque partie ${ }^{3}$ : cet élément, trop souvent oublié par les auteurs, leur donne aussi un caractère

H. LEVY-BRUHL, op. cit., (L'Ethnologie juridique)., 1162.

"The song duels are juridical instruments insofar as they do serve to settle disputes and restore normal relations between estranged members of the community. One of the contestants receives a "judgment" in his favor. There is, however, no attempt to mete justice according to rights and privileges defined by substantive law. It is sufficient that the litigants (contestants) feel relieved - the complaint laid to rest - a psychological satisfaction attained and balance restored. This is justice sufficient unto the needs of Eskimo society as the Eskimos conceive it. It is, so far as here achieved, an element in which "higher" cultures often fail" (E. ADAMSONHOEBEL, op. cit., 98-99). Dans le même sens, cf. I. KLEIVAN, op. cit., 9 : "This material (of West Greenland) provides evidence that satirical song duels were not, as they are usually considered to be, primarily a punitive measure available to persons with a specific grievance, but were associated with more permanent conflict-laden relationships. By bringing inter-personal and intergroup antagonisms out into the open in this formalised way, more overt forms of hostility were avoided" (Cf. aussi, ibid., 12).

3 Cf. nos analyses, supra, pp. 67-68, 72. 
éminemment juridique et judiciaire, qui cependant, ne réside pas dans l'attribution d'une peine afflictive à une des parties ${ }^{1}$.

[p. 95]

On évite ainsi que les antagonismes s'enveniment en conflits ouverts, dangereux pour l'ensemble du groupe ${ }^{2}$. La preuve en est que, sur la Côte Ouest du Groënland, tout au moins, ces compétitions de chants mettent très souvent en présence des individus appartenant à des communautés différentes ${ }^{3}$, rassemblées par la nécessité d'une chasse collective ${ }^{4}$ : en l'absence d'une autorité politique, la compétition de chants permet de réduire les conflits éventuels entre les groupes forcés de coexister pour un temps donné. C'est ainsi qu'en dernière analyse, cette forme de duel judiciaire peut aussi revêtir un aspect politique.

En conclusion, nous voudrions mettre l'accent sur la polyvalence des compétitions de chants: nous avons tenté de démontrer qu'elles pouvaient revêtir une forme juridique, et constituer de véritables duels judiciaires, compte tenu de la signification particulière de ces concepts quand on les applique à des sociétés archaïques. Mais il ne s'agit là que d'un des aspects de ces compétitions. Nous avons vu qu'elles ont aussi une valeur psychothérapeutique, politique, et sont une espèce de divertissement. Elles peuvent aussi intervenir, non dans un cadre conflictuel, mais pour préserver des relations d'amitié ${ }^{5}$ : chacun sait que la même observation, selon la forme dans laquelle elle est énoncée, peut prévenir un conflit ou, au contraire, l'ouvrir. Mais, dans les deux cas, l'objectif ultime reste bien le respect de l'intérêt public, que manifeste dans les formes que nous avons examinées, l'intervention directe et codifiée de la collectivité.

[p. 96]

Cette diversité peut aussi s'apprécier d'un point de vue anthropogéographique : les duels judiciaires revêtent des formes et ont un contenu diffé-

\footnotetext{
Nous sommes en ce sens d'accord avec I. KLEIVAN lorsqu'elle porte une accusation d'ethnocentrisme contre certains auteurs qui fondaient la judiciarité de ces compétitions sur ce seul caractère : "The words used by these two authors (Glahn and Thorhallesen) show clearly that they compare the institution of satirical singing in Greenland with what they consider to be its equivalent in their native countries, Denmark and Iceland respectively. Because the Europeans had this model on which to base their appraisal, the satirical songs were considered to be juridical songs, and more importance was attached to their function as punitive remedies for violation of the norms than to their role in preventing a breach in social relations" (I. KLEIVAN, op. cit., 13).

Cf. I. KLEIVAN, op. cit., 16.

Cf. I. KLEIVAN, op. cit., 12-13.

Ibid., 12.

"Friendships are vulnerable, if expectations are not lived up to. Envy can arise if one has not done as well as one's friend, or one can feel the need to boast about one's feats. In a satirical song, much can be said without spoiling the friendship. Joking relationship thus serves both to avert open conflicts and to strengthen friendships". (I. KLEIVAN, op. cit., 20. Dans le même sens, cf. aussi ibid., 33).
} 
rent selon le type de société inuit qui les pratique. C'est ce problème que nous voudrions étudier dans le dernier paragraphe de ce chapitre.

\section{C) ÉLÉMENTS D'UNE ANTHROPO-GÉOGRAPHIE DES COMPÉTITIONS DE CHANTS}

Retour à la table des matières

On a déjà noté la diversité des sociétés inuit et l'influence de cette diversité sur leurs structures sociales et le contenu de leurs normes juridiques ${ }^{1}$. Cette diversité, à notre sens, provient de la différence de leur contexte économique et de leur histoire ${ }^{2}$. J. Malaurie a ainsi montré que, parmi les sociétés inuit, c'est chez les populations behringiennes, favorisées par leur économie baleinière et leur force démographique, que la guerre et l'esclavage se sont manifestés avec le plus de force, avec une vigueur inconnue dans d'autres régions arctiques où l'environnement réduisait considérablement plus la marge de survie du groupe ${ }^{3}$. Comme l'écrit l'auteur :

"...l'agressivité déclarée dégénérant en conflit organisé implique en effet des moyens économiques, une certaine densité démographique, une organisation sociale élaborée" ${ }^{4}$.

Il est tentant d'essayer de voir si les modes de règlement pacifiques des conflits - et plus particulièrement la compétition de chants, qui est le sujet de cette partie de notre étude - obéissent à cette hiérarchie démo-économique des sociétés inuit.

H. König a dressé une carte de localisation des diverses formes de duels judiciaires qui a été reproduite à la page 79. D'après ces données, [p. 97] il semblerait que les compétitions de chants soient pratiquées dans l'Arctique oriental (Groënland Est, Ouest, Nord-Ouest, Cumberland Sound et Côtes du Labrador) et occidental (région de Behring et Nord-Ouest Alaska de Kotzebue à Cook Inlet, Îles Aléoutiennes). König ne les signale en revanche nulle part dans l'Arctique Central

Cf. J. MALAURIE, op. cit., (Les civilisations esquimaudes...), 46.

On ne peut en ce sens que souscrire à l'opinion de J. MALAURIE, qui affirme : "... histoire close ne veut pas dire histoire uniforme. Notre ignorance ne peut s'accommoder de telle conclusion. L'histoire est globale et doit expliquer. Les peuples archaïques ont une histoire. Des changements de climat, des crises démographiques, des famines, des contacts culturels aussi, ont fait connaître à ces populations des temps d'expansion, de réduction et d'étiage démographique, des crises techniques". (J. MALAURIE, op. cit., (Raids et esclavage...), 26.

Ibid., 8.

Ibid., 27. 
canadien. Il semble bien que cette carte pèche par omissions ${ }^{1}$. S'il est vrai qu'on trouve effectivement des duels de chants dans toutes les régions citées par König, plusieurs observations les attestent dans l'Arctique Canadien, à des degrés divers : ils sont, en effet, pratiqués par les Inuit Netsilik ${ }^{2}$ et les Caribou ${ }^{3}$. Mais ils sont moins fréquents chez les Netsilik ${ }^{4}$ que sur la Côte Ouest du Groënland, et encore plus rares chez les Caribou ${ }^{5}$. En revanche, D. Jenness semble conclure pour la négative en ce qui concerne les Inuit du Cuivre ${ }^{6}$. Remarquons aussi que certaines diversités régionales apparaissent au Groënland ${ }^{7}$ : sur la Côte Ouest, les compétiteurs usent volontiers de l'ironie à l'égard d'eux-mêmes, au contraire de ce qui se passe sur la Côte Est ${ }^{8}$; E. Holtved prétend que, chez les Inuit Polaires, les compétitions de chants n'interviennent que comme des distractions entre amis, mais jamais pour régler des conflits ${ }^{9}$. Cette observation est implicitement démentie par P. Freuchen, qui rapporte que de tels duels ont lieu lorsqu'un individu en offense un autre, ou se montre paresseux ou voleur ${ }^{10}$.

\section{[p. 98]}

Quoi qu'il en soit du cas du Groënland, la gradation signalée par G. Van den Steenhoven pose déjà des problèmes quant à sa signification, dans la mesure où l'on suppose le critère économique déterminant : puisque les sociétés de l'Ouest Groënlandais sont plus riches que les Netsilik, eux-mêmes plus privilégiés que les Caribou, on s'attendrait à ce que ces compétitions soient les plus fréquentes chez les Caribou, puis les Netsilik et, enfin, l'Ouest Groënlandais. Or, c'est exactement le contraire qui se produit... On pourrait alors essayer d'incriminer un autre facteur : le degré de structuration "politique" de la collectivité, la fréquence des compétitions de chant, manifestation de l'action directe du groupe, correspondant à ce degré ${ }^{11}$. Mais, là encore, il ne peut s'agir que d'un faux critère : nous avons vu que, si on trouve effectivement des compétitions de chants dans les sociétés fortement hiérarchisées de la région de Behring et des îles Aléoutiennes, elles sont

Cf. supra, pp. 77-78.

Cf. G. VAN DEN STEENHOVEN, op. cit., (Legal concepts...), 30-36. A. BALIKCI, op. cit. (The Netsilik Eskimo), 185-189.

Ibid., 30.

4 Chez les Netsilik, les Utkuhikjalingmiut, les duels à coups de poing sont beaucoup moins fréquents que chez les autres groupes Netsilik, car ils considèrent que ce n'est pas un critère suffisant pour prouver la valeur d'un homme (Cf. K. RASMUSSEN, op. cit. (The Netsilik Eskimo), 489).

Cf. G. VAN DEN STEENHOVEN, op. cit. (The Eskimos), 354.

Cf. D. JENNESS, op. cit., 94.

M. MAUSS prétendait abusivement, qu'au Groënland, la compétition de chants et la danse du tambour étaient, en hiver, les seuls modes de sanction (Cf. M. MAUSS, "Essai sur les variations saisonnières des sociétés eskimos", dans L'Année sociologique (1905), 111).

Cf. E. ADAMSON-HOEBEL, op. cit., 96.

Cf. E. HOLTVED, op. cit., 153-154.

Cf. P. FREUCHEN, Book of the Eskimos (Greenwich, Conn., 1961), 124.

11 Par exemple, G. VAN DEN STEENHOVEN, op. cit. (The Eskimos), 350, note que, d'une façon générale, la communauté s'exprime d'une façon plus active chez les Netsilik que chez les Caribou. 
encore plus fréquentes sur la Côte Est du Groënland, caractérisée par une structure socio-politique beaucoup plus "archaïque" et diffuse ${ }^{1}$. En tout cas, un fait demeure certain : les compétitions pacifiques, et plus particulièrement les duels de chants, sont attestées dans les sociétés inuit démo-économiquement pauvres ${ }^{2}$. Donnons-en quelques exemples :

- Les Inuit d'Ammassalik (Côte Est du Groënland), qui vivent dans des conditions très dures et dont les communautés sont de faible effectif [p. 99] numérique, sont ceux qui ont le plus développé les compétitions de chants et de tambour pour le règlement des conflits ${ }^{3}$. Ces compétitions sont un des moyens d'expression privilégiés de la communauté ${ }^{4}$, qui exerce à cette occasion une véritable autorité judiciaire ${ }^{5}$. Les duels peuvent se répéter durant des années ${ }^{6}$, les individus peuvent être propriétaires de leurs chants ${ }^{7}$.

- Chez les Caribou, les compétitions de chants sont très rares ${ }^{8}$. J. Gabus note cependant que ces Inuit recourent aux chants satiriques ${ }^{9}$.

- Nous avons vu qu'elles existaient aussi très probablement chez les Inuit Polaires de Thulé ${ }^{10}$.

Il n'est pas inintéressant de noter que certaines des populations pratiquant les compétitions de chants appliquent des principes plus souples qu'ailleurs en matière de répression de l'homicide :

- Sur la Côte Est du Groënland, alors que partout ailleurs (sauf chez les Inuit du Cuivre et d'Iglulik) la vengeance familiale est la sanction quasi générale du meurtre, l'homicide n'est vengé que selon la force des parties en présence ${ }^{11}$.

1 J. MALAURIE (op. cit. (Raids et esclavage...), 27) s'est heurté au même genre de difficultés en constatant que, logiquement, on aurait pu s'attendre à retrouver chez les populations de la Côte Sud-Ouest du Groënland, caractérisées par l'abondance économique (chasse à la baleine) et de gros effectifs démographiques, un niveau d'activités guerrières comparable à celui des sociétés behringiennes. Or, il n'en est rien, en dépit de circonstances démo-économiques identiques. L'auteur se tourne alors vers une explication d'ordre historique : "...peut-être peut-on éclairer le mystère en considérant que ce secteur d'occupation peu ancienne (le Sud-Ouest Groënlandais) a bénéficié d'une unité linguistique, alors qu'en Alaska, un temps historique plus long s'est traduit très tôt par une balkanisation linguistique se soldant par une méfiance entre isolats et des guerres endémiques" (J. MALAURIE, ibid.) L'explication est, certes, pertinente s'agissant du phénomène guerrier, mais nous ne saurions l'utiliser quant à notre objet.

Cf. J. MALAURIE, op. cit., (Les civilisations esquimaudes...), 46 ; (Raids et esclavage...), 27.

Cf. J.W. LAPIERRE, op. cit., 496, n. 249.

Cf. C.C. HUGHES, op. cit., 362-363, 371.

Ibid., 371. Dans le même sens, cf. R. GESSAIN, op. cit., 60 ; P.E. VICTOR, op. cit., 165, n. 1.

Contra, cf. M. GLUCKMANN, op. cit., 306, qui y voit surtout un divertissement social.

Cf. R. GESSAIN, op. cit., 57 ; M. GLUCKMANN, op. cit., 305.

Cf. R. GESSAIN, op. cit., 42.

Cf. G. VAN DEN STEENHOVEN, op. cit. (Legal Concepts...), 30 ; (Caribou Eskimo...), 6.

Cf. J. GABUS, op. cit., 140.

Cf. supra, p. 97.

1 Cf. E. ADAMSON-HOEBEL, op. cit., 87. Contra, cf. K. RASMUSSEN, op. cit. (Life and doings...), 85. 
- Chez les Inuit Caribou, le meurtre n'entraîne pas de représailles si l'ensemble de la communauté donne raison au meurtrier ${ }^{1}$.

- Chez les Netsilik, la vengeance familiale connaît des limitations qui ont pour but d'assurer la préservation du capital humain : quand sur 20 ou 40 vengeurs, 4 individus ont été blessés ou tués, les hostilités [p. 100] s'arrêtent ${ }^{2}$ : les jeunes et bons chasseurs, au cours des raids de vengeance, sont tout particulièrement protégés par leurs compagnons ${ }^{3}$.

Tout ceci incline à penser que ces sociétés, plus menacées que d'autres en raison de leur faible marge de survie, recourent volontiers à des solutions évitant de trop grosses pertes en vies humaines : compétition de chants, limitations de la vengeance privée. Alors, qu'au contraire, les sociétés riches connaissent un véritable déchaînement des activités guerrières, entraînant une peur généralisée ${ }^{4}$. Il reste, cependant, que l'énigme du déterminant principal, que nous constations plus haut ${ }^{5}$, est loin d'être totalement élucidée, puisqu'il faudrait expliquer l'existence des compétitions de chants dans des sociétés plus riches (Behring et Alaska), et leur absence chez les populations guère moins pauvres (Inuit du Cuivre). Si l'économie n'est donc pas le critère absolu - bien que ce facteur demeure à notre sens déterminant - sans doute faudrait-il chercher dans l'histoire de ces sociétés la raison de ces anomalies régionales. Faute d'éléments, nous ne pouvons ici qu'exprimer un vœu pieux, en souhaitant que d'autres chercheurs puissent répondre à notre attente.

On voit donc que, dans tout ce qui précède, le champ de l'hypothèse est encore largement ouvert. Nous n'avons pas voulu, pour notre part, donner une allure trop systématique à nos réflexions : le droit classe, mais il fige aussi, et les mailles de son filet peuvent laisser échapper la réalité de la vie sociale. Nous espérons, en tout cas, avoir convaincu le lecteur de la subtilité des processus de sanction du droit dans les sociétés Inuit, notamment dans leurs aspects socio-psychologiques. Le juriste sera peut-être gêné par l'éclatement de certains de nos concepts qu'une telle démarche implique, alors que l'historien, plus habitué à l'effort d'adaptation que lui impose la prise en compte d'époques déterminées par d'autres principes que les nôtres, aura reconnu en ces sociétés certains traits de notre Antiquité ou de notre passé médiéval. Gageons, cependant, qu'à tous sera apparu, dans le champ nécessairement déterminé de notre étude, que [p. 101] dans des sociétés où font défaut les formes les plus élémentaires des institutions étatiques, l'intérêt public peut, néanmoins, être encore plus fortement protégé qu'ailleurs, où ne manque pas un appareil répressif et institutionnel spécialisé. On conviendra également qu'alors que certains penseurs liaient abusivement le phénomène du droit (et de sa sanction) à l'existence de l'État, on voit le premier fort bien se passer de l'autre : la

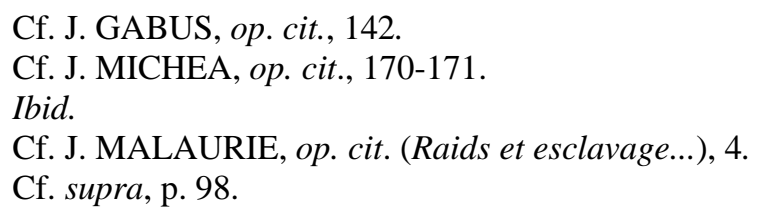


vision paradisiaque de sociétés où tout est adhésion spontanée en l'absence d'un État par nature coercitif appartient au monde séduisant et souvent fallacieux de l'hypothèse ou de l'acte de foi. 
[p. 103]

\title{
CONCLUSION DE LA $1^{\text {re }}$ PARTIE
}

\author{
"Il faut éclairer l'Histoire par les \\ lois, et les lois par l'Histoire". \\ Montesquieu
}

\section{DE L'HISTOIRE DU DROIT À L'ETHNOLOGIE : MOI ET L'AUTRE}

\section{$\underline{\text { Retour à la table des matières }}$}

C'est le propre des objets de luxe d'être réservés à une minorité, et de procurer du plaisir sans avoir de réelle utilité. À voir le sort fait à l'ethnologie - sans même parler de sa branche juridique - dans l'enseignement supérieur français à l'heure actuelle, tout porte à croire que beaucoup continuent à n'y voir qu'une curiosité marginale, dont l'exotisme excuse peu la gratuité. Nous vivons pourtant une époque où on nous parle beaucoup de "mondialisation" : mais la vision de l'Autre se heurte cependant à des barrières mentales toujours bien dressées. Nous n'avons guère changé depuis le $\mathrm{XVI}^{\mathrm{e}}$ siècle européen, qui vit en même temps la naissance de l'idéal humaniste, et le début des grandes colonisations, avec leur cortège d'ethnocides. Du touriste naïf, qui réduit la différence au pittoresque, au scientifique enfermé dans des concepts ethnocentristes, l'attitude est en fin de compte la même. Et pourtant, dans un monde où, de plus en plus, la spécialisation tient lieu de formation intellectuelle, le visage de l'Autre nous renvoie sans cesse à notre propre image, pour peu qu'on veuille bien le dévisager sans le défigurer. L'historien sait bien, lui aussi, que sa démarche n'est pas gratuite : comme l'a si bien dit André Malraux, "ceux qui prétendent ignorer le passé se condamnent à le revivre". Mais l'Histoire appréhende l'Autre dans son passé. Alors que l'ethnologue a pour champ d'étude le présent passé : même altérées par l'acculturation, les populations qu'il étudie sont - pour combien de temps ? - encore vivantes : mais leur éloignement géographique les repousse également loin dans notre passé. Et c'est ici, en ce point où le temps et l'espace se conjuguent pour donner au visage de l'Autre cette altérité que s'efforce de scruter l'ethnologie, que cette dernière et 
l'Histoire pourraient se rencontrer ${ }^{1}$. Nous avons, dans cette étude et ailleurs, essayé de montrer, sans [p. 104] tomber dans les excès d'un comparatisme abusif et de ce fait insignifiant, une certaine identité dans les structures juridiques des Inuit et celles de peuples de notre passé européen ${ }^{2}$ Il est hautement improbable qu'il ne s'agisse là que de pures coïncidences. D'une part, parce qu'on peut penser que certaines sollicitations de l'environnement produisent chez des hommes séparés par le temps et l'espace des besoins souvent voisins, qu'ils s'efforcent de satisfaire par des productions socio-juridiques dont la parenté n'est que le reflet d'une identique nécessité ${ }^{3}$. Ensuite, parce que l'apport des civilisations nordiques dans la formation de la culture européenne a été systématiquement sous-évalué. Le prestige de l'antiquité gréco-latine, sa valorisation - non dépourvue d'ambiguïté - par l'Église, des sources écrites et relativement accessibles, l'acculturation de ces peuples germains et scandinaves, sont autant de facteurs qui expliquent que, dans le creuset que fut l'Europe du IV au $\mathrm{X}^{\mathrm{e}}$ siècle, l'élément boréen ait été compté pour peu. Certains - peu nombreux - s'en sont aperçus, et ils sont trop rares pour qu'on ne les cite pas :

"L'étude des grandes migrations qui ont secoué l'Occident depuis le II siècle de notre ère constitue sans doute l'un des dossiers les plus importants de la chute de l'Empire Romain et de la fin du monde antique. Mais elle est intrinsèquement lourde d'une signification beaucoup plus riche. Elle concerne, en effet, la couche d'humus ethnique qui s'est déversée sur le vieux sol démographique romano-grec usé. L'Europe [p. 105] chrétienne et tout ce qu'on entend par civilisation occidentale ont poussé sur ces deux strates superposées, sinon intimement mêlées. Certes, le Haut Moyen-Âge a subi aussi de grands remuements de peuples, mais qui - les Scandinaves exceptés - n'ont déposé sur l'Europe que de l'écume. L'Occident chrétien d'aujourd'hui est né au Bas-Empire d'une romanité adultérée de germanisme. L'histoire de

1 Ce qui suppose aussi, comme l'a bien vu J. POIRIER, que les deux disciplines empruntent l'une à l'autre certains de leurs caractères, l'Histoire devenant plus culturelle (au sens large du terme) et l'ethnologie plus diachronique (l'ethnologie concernant un ensemble humain, elle ne peut décrire un éternel présent, qui n'appartient qu'au mythe). (Cf. J. POIRIER, "Ethnologie diachronique et histoire culturelle", dans Ethnologie Générale, op. cit., 1457-1460). Cf. aussi : Ethnologie et histoire, Forces productives et problèmes de transition (Paris, 1975).

Cf. N. ROULAND, op. cit., (Approche du phénomène juridique).

Cf. pour l'étude des sociétés chasseresses, les très riches données comparatives fournies par : Man, the Hunter (edited by R.B. LEE and I. DE VORE ; Chicago, 1975). J. GAUDEMET a fort bien exprimé le point de vue auquel nous souscrivons pleinement : "Il est sans doute toujours possible et souvent facile de relever des analogies de détail entre sociétés profondément différentes et séparées par les siècles et les océans. Un tel catalogue n'est qu'un jeu, brillant et sans conséquences. Mais, lorsque les analogies touchent à des données fondamentales de l'organisation sociale (...) elles sont plus qu'une convergence due au hasard". (J. GAUDEMET, "Droit Romain et coutumes malgaches", dans Mél. H. Lévy-Bruhl, (Paris, 1959), 371). 
cette rencontre rude et de cet accouplement difficile a pour substance essentielle le devenir de ce qui fut alors engendré" ${ }^{1}$.

Or - et il est particulièrement important de le noter - l'ethnologue fait ici écho à l'historien du droit : J. Malaurie a sans cesse souligné dans ses travaux que l'apport des civilisations nordiques à notre culture devait être réévalué :

- "De plus en plus (...) les sciences de l'homme soupçonnent que le rôle des cultures hyper-boréales dans la formation des civilisations classiques a été sous-estimé. La plupart des grands mouvements d'invasion qui ont, dans les temps anciens, submergé l'Europe, semblent avoir eu pour aire de départ les zones où paraît s'être constituée, entre autres, l'ethnie esquimaude. Dans cette perspective, les cultures arctiques d'aujourd'hui pourraient fort bien constituer le dernier vestige, l'ultime expression de très anciens systèmes de valeurs, notamment sur le plan de la propriété, renvoyant à une conception déterminée de l'homme et du groupe, et partiellement incorporés, par l'action même des envahisseurs, dans l'infrastructure profonde des sociétés des régions tempérées" ${ }^{2}$.

- "La civilisation européenne elle-même n'a pas échappé à ces apports asiatiques et boréaux qui en constituent le substrat le plus profond. Et l'on ne saurait ainsi minimiser le rôle important qu'à travers les migrations doriennes et scythes les peuples nordiques ont joué dans les cultures proto-méditerranéennes" ${ }^{3}$.

- "Il est remarquable que l'historien et l'ethnologue, dans leurs exposés généraux, ignorent presque toujours les sociétés hyperboréennes, ce qui est d'autant plus fâcheux que ces dernières sont l'illustration unique et [p. 106] vivante de ce qui a été en Europe les trois quarts de notre histoire ; elles permettent de saisir sur le vif des passages sociologiques fondamentaux : famille, clan, rang, classe" ${ }^{4}$.

La dette de la culture occidentale aux civilisations nordiques dépasse d'ailleurs les frontières de l'Europe :

"Si l'on note que les deux tiers de l'Amérique du Nord, le tiers de l'Eurasie ont été englacés, si l'on considère que tous les peuples ayant im-

L.R. MENAGER, La chute de l'Empire romain (C.R.D.P., Marseille, 1966), Ch. II, p. 1.

J. MALAURIE, op. cit. (Spécificité des sociétés esquimaudes...) 11-12.

J. MALAURIE, Préface à J.L. GIDDINGS, 10000 Ans d'Histoire arctique (Paris, 1973), 19. Dans le même sens, cf. J. MALAURIE, Thèmes de recherche géomorphologique dans le Nord-ouest du Groenland (Paris, 1968), 9.

4 J. MALAURIE, op. cit. (Raids et esclavage...), 4. 
migré en Amérique ont connu, sur le pont de Behring et en Alaska, un temps arctique prolongé lors de leurs déplacements, temps dont ils ne peuvent manqué d'avoir gardé des traces dans leurs systèmes de vie et leurs croyances, l'on saisit qu'une partie de l'humanité a été dans sa haute histoire un peuple du froid, et l'on mesure la nécessité d'une appréciation nouvelle du fait boréal dans l'histoire de l'évolution de la vie et le déroulement des civilisations ${ }^{1}$.

Arrêtons là ces citations, suffisamment éloquentes : le froid a beaucoup moins figé qu'on ne le pense l'histoire des sociétés arctiques et nordiques. Non seulement cette histoire n'est pas close d'un point de vue interne, puisqu'informée, selon les lieux, par les contacts culturels et les changements climatiques, mais elle déborde largement - et plus qu'on ne le soupçonne - sur notre passé. Bien sûr, l'inventaire de ces apports reste quasiment à dresser. Encore faut-il en sentir la nécessité, et trop peu de voix s'élèvent pour la souligner.

Mais il est un autre point que celui de la formation de notre civilisation où l'intérêt du juriste pour l'ethnologie - qu'il soit historien ou positiviste - devrait être éveillé : non seulement, en effet, l'ethnologie peut éclairer l'histoire, mais aussi le droit. Or, au ciel des disciplines considérées comme fondamentales, l'étoile de l'ethnologie juridique ne brille pas d'un éclat particulier et rejoint dans ce déclin l'astre de l'histoire, dont l'éclipse semble se confirmer alors que, paradoxalement, les "sciences humaines" connaissent une vogue sans précédent. Curieuses sciences humaines qui nient ainsi l'altérité de l'Homme dans l'espace et dans le temps ... L'aspect trop souvent rhétorique de ces prétendues sciences ne viendrait-il pas justement en partie de cette méconnaissance fondamentale, qui participe plus d'un jugement a priori que d'une attitude véritablement scientifique ? Comme celle du monde physique, la connaissance de l'homme ne peut se baser [p. 107] que sur des faits concrets : sans quoi on se perdra toujours plus foin dans l'univers incertain du logos, et on aura au bout du compte une nouvelle métaphysique, sans doute encore moins fondée que celle que le matérialisme prétendu détruire. Encore au moins la métaphysique religieuse avait-elle pour mérite de dire clairement qu'elle se situait au-delà des preuves rationnelles, dans le domaine de l'acte de foi... Mais que l'on sache, les sciences humaines, au moins officiellement, se veulent laïques et objectives. Encore faudrait-il pour cela que les diverses sociologies et politologies, avant de formuler des dogmes, les appuient sur plus de faits : or l'ethnologie (comme l'histoire) nous en fournissent. C'est donc dire qu'une véritable science $d u$ droit, pour nous cantonner au domaine qui est plus précisément le nôtre, ne saurait nullement borner son étude aux normes juridiques du droit positif des sociétés dites "modernes". Sans quoi, on se condamne à n'appréhender qu'une partie du champ juridique, et à passer sous silence la fonction socio-politique du droit. Nous pensons, en effet, que l'examen des similitudes et différences - dues au

J. MALAURIE, op. cit. (Préface à 10000 Ans d'Histoire arctique...) 11. 
temps ou à l'espace - des systèmes juridiques constitue la voie privilégiée ${ }^{1}$ par laquelle on peut saisir la liaison du droit au social, ainsi que sa véritable fonction : une étude chronologiquement ou spatialement limitée ne peut conduire qu'à une vision abstraitement statique, à un point de vue particulier. Il ne faut pas se dissimuler les difficultés de l'entreprise : la dilatation des concepts qu'entraîne l'altérité des situations envisagées rend l'approche ardue. (Nous en avons eu un exemple dans les lignes qui précèdent en tentant d'analyser la notion et les modalités de la sanction juridique dans des sociétés différentes des nôtres). La vision de l'Autre est donc essentielle à la compréhension du Moi : on retrouve, sur le plan purement intellectuel, le précepte posé au niveau moral par les grandes religions.

Or, que constatons-nous ? Qu'à cette exigence fondamentale répond une orientation exactement inverse. Les matières historiques sont progressivement effacées des programmes de l'enseignement secondaire et supérieur ; quant à l'ethnologie, elle n'est qu'un luxe, de plus en plus cher, et donc de plus en plus rare. Une telle évolution n'est innocente ni dans ses tenants, ni dans ses aboutissants ${ }^{2}$.

[p. 108]

Il serait cependant injuste et inexact de ne pas mentionner l'existence de tout un courant d'auteurs bien conscients de la nécessité d'une approche s'exprimant dans les termes que nous rappelions plus haut. Mais une certaine tradition, ainsi que la conjoncture socio-économique propre à notre temps empêchent que leurs voix soient entendues comme elles le mériteraient. Attardons-nous un peu sur ces quelques points.

Tout d'abord, quelques mots sur les idées exprimées par ces différents auteurs. Certains, comme R. Aron, mettent à juste titre l'accent sur les défauts d'un certain enseignement pour expliquer la désaffection éprouvée actuellement à l'égard de ces matières : si certains sont indifférents à l'étude du passé, c'est en partie à cause de l'ethnocentrisme des historiens qui ont trop privilégié l'étude de l'antiquité gréco-latine, dans laquelle ils voyaient la seule source de notre passé. Cette attitude n'est cependant pas entièrement négative car, par réaction, les contempteurs de cette histoire traditionnelle font volontiers appel à l'ethnologie pour l'étude des civilisations extérieures à l'Occident ${ }^{3}$.

Cf. T.S. BOTTOMORE, op. cit., 53-56.

Nous faisons référence à la situation actuelle en France.

"... les historiens au sens étroit et académique du terme, tout en reconnaissant pour la plupart le caractère européo-centrique de la connaissance historique transmise par les universités à l'époque même où s'épanouissait la conscience historique, tout en acceptant d'enlever à l'antiquité gréco-latine ou au passé occidental sa valeur exemplaire, continuent de privilégier en fait ceux qui passent pour nos ancêtres. La prétendue indifférence au passé s'accompagne bien souvent d'un intérêt accru pour les civilisations étrangères que la révolte contre l'Occident et la colonisation a rendues à elles-mêmes (...). Quant à l'autre forme du refus de la culture historique, celle qui dérive d'un souci exclusif du présent (...), elle se fonde, implicitement au moins, sur le postulat de la singularité de notre destin". (R. ARON, Postface), L'historien entre l'ethnologue et le futurologue (Paris, 1972), 267. 
C. Lévi-Strauss, à sa façon, plaide pour un rapprochement entre l'histoire et l'ethnologie: il voit entre elles de nettes différences de démarches, mais il n’"évacue" pas l'Histoire, comme on l'a beaucoup trop souvent dit et écrit :

"...elles (l'ethnologie et l'histoire) se distinguent surtout par le choix de perspectives complémentaires : l'histoire organisant des données par rapport aux expressions conscientes, l'ethnologie par rapport aux conditions inconscientes de la vie sociale" ${ }^{1}$.

[p. 109]

Il est difficile de souscrire entièrement à ce point de vue : l'histoire prend de plus en plus en compte les attitudes mentales et les données de l'inconscient dans l'appréciation portée par les hommes sur le réel et, d'autre part, l'ethnologie ne saurait se réduire à l'exploration de l'inconscient social. Cependant, l'auteur poursuit :

"Tout bon livre d'histoire...est imprégné d'ethnologie (...). Véritable Janus à deux fronts, c'est en tout cas la solidarité des deux disciplines qui permet de conserver sous les yeux la totalité du parcours" 2 .

D'autre part, J. Poirier a bien montré que l'histoire - telle qu'elle est faite $a c$ tuellement : il est trop facile de condamner une discipline sur son passé, ce qui est en soi-même une attitude anti-historique - est très proche de l'ethnologie ${ }^{3}$ :

"...à partir du moment où, par un mouvement convergent, l'ethnologie est devenue diachronique et où l'histoire est devenue culturelle, la situation change. Aujourd'hui, en effet, l'histoire a rencontré l'ethnologue (...) au terme de la recherche... l'histoire et l'ethnologie vont s'unir dans la même entreprise de reconstitution totale qui implique la mise à nu des structures des squelettes sociaux" ${ }^{4}$.

De l'ethnologie à la sociologie la différence n'est pas d'essence : les phénomènes observés sont les mêmes, seul de champ d'étude diffère ${ }^{5}$ : il est donc logique,

C. LÉVI-STRAUSS, Anthropologie structurale (Paris, 1958), 24-25.

Ibid., 31-32.

Le récent livre d'E. LEROY LADURIE, Montaillou, village occitan (op. cit.), est un exemple de ce que peut donner l'union entre la vision ethnologique et historique.

J. POIRIER, op. cit. (Histoire culturelle et ethnologie diachronique), 1457, 1459.

Cf. J. CARBONNIER, op. cit. (Sociologie Juridique), 29. 
là encore, et contre tout un courant hostile à l'histoire, de trouver des auteurs favorables au contraire à une collaboration entre l'histoire et la sociologie. Ainsi T.S. Bottomore écrit que :

"...plus les distinctions qui rendent compte du travail propre à l'historien et au sociologue deviennent minutieuses, plus il apparaît clairement qu'il est impossible de séparer radicalement historiographie et sociologie (...). Il importe beaucoup, pour le progrès des sciences sociales, que les deux restent intimement en [p. 110] rapport, et que chacune puisse largement puiser dans l'autre..." ${ }^{1}$.

Certains, comme P. Veyne, vont même jusqu'à penser que, loin de rendre l'histoire inutile, l'ethnologie et la sociologie manifestent sa nécessité en ce qu'elles sont elles-mêmes des formes particulières d'histoire :

"La première convention nous a habitués à opposer le présent, qui serait la chose même, et le passé, qui est affecté d'un indice historique qui le rend à demi irréel. Cette fausse opposition est à l'origine de deux pseudo-sciences, la sociologie et l'ethnographie, qui se partagent l'histoire des civilisations contemporaines, l'une prenant celle des civilisés et l'autre celle des primitifs (...) n'étant pas affectées de l'indice historique, ces deux disciplines évoluent en liberté dans un éternel présent ${ }^{2}$.

(...) Or cette histoire complète rend inutile la sociologie : elle fait tout ce que fait celle-ci et le fait mieux qu'elle. La frontière entre les deux disciplines est déjà devenue insaisissable ; depuis un bon tiers de siècle, les historiens font, sous le nom d'histoire non événementielle, ce qu'on aurait appelé auparavant de la sociologie ${ }^{3}$ (...) la sociologie naît et vit des incomplétudes de l'histoire... Il convient donc que les historiens prennent conscience que la sociologie est de l'histoire qu'ils négligent d'écrire, et dont l'absence mutile celle qu'ils écrivent, et que les sociologues et ethnographes comprennent qu'ils ne peuvent faire plus scientifique que les historiens" ${ }^{4}$.

Le ton peut surprendre par sa vigueur : il vient après tout en réponse à des attaques non moins violentes formulées contre l'histoire. Il n'en soutient pas moins des arguments épistémologiques dont la justesse, en dépit de certaines outrances marginales, nous paraît pour l'essentiel évidente. (Nous nous en sommes d'ailleurs

T.B. BOTTOMORE, op. cit., 77.

P. VEYNE, Comment on écrit l'histoire (Paris, 1971), 334.

Ibid., 339.

Ibid., 332. 
expliqués dans d'autres travaux ${ }^{1}$ ). Dans [p. 111] le champ précis du juridique, il est d'ailleurs frappant de constater combien la démarche du sociologue et de l'historien du droit se confondent dans leur objet. D'après Roscoe-Pound, en effet, la sociologie du droit doit avoir pour finalité essentielle l'étude de :

- l'organisation juridique, c'est-à-dire un régime qui ajuste les relations et ordonne la conduite par l'usage systématique de la force d'une société politiquement organisée.

- Les principes et les règles faisant autorité dans le règlement des disputes dans une société, un code de préceptes basé sur un idéal commun.

- La procédure judiciaire et la procédure administrative ${ }^{2}$.

Quel historien du droit ne reconnaîtrait pas là l'essentiel de sa tâche ? La raison profonde de cette convergence tient au fait que l'historien et le sociologue du droit étudient tous deux le droit non en tant qu'art, mais comme science : alors que le juriste dogmatique est forcément intégré à son système national - et donc en proie à l'ethnocentrisme - et ne peut porter sur lui qu'un regard critique fort limité, l'historien, le sociologue, et l'ethnologue de droit peuvent eux adopter une attitude réellement scientifique, ne serait-ce que parce qu'à l'instar des sciences expérimentales, ils trouvent dans leur discipline la séparation radicale entre l'observateur et la matière observée ${ }^{3}$. Nous verrons plus loin ${ }^{4}$ que la faculté critique que, par nature et épistémologiquement, ils peuvent et doivent ainsi exercer, explique en partie la suspicion plus ou moins avouée dans laquelle ils sont tenus.

On voit donc, d'après ce qui précède, que l'étude scientifique du droit implique l'élargissement de l'objet de sa recherche, l'abandon [p. 112] d'habitudes mentales trop étroites, le recours à des types d'explication autres que ceux hérités de la pensée juridique classique. L'enjeu est d'importance : ou bien l'on continuera à camper sur les positions ébranlées du dogmatisme et de l'idéalisme juridiques, auquel

1 "L'histoire narrative ne dessine, en effet, que les coutures, mais la trame et les motifs du tissu sont faits du non-événementiel... La description a été longtemps prise pour l'explication : le déterminisme linéaire d'une chronologie aux origines incertaines et à l'aboutissement inconnu suffisait à dispenser chroniqueurs et hagiographes de l'étude d'une causalité liée plus aux rapports structurels des phénomènes qu'ils décrivaient qu'à leur simple succession. Les historiens tentent, au contraire, de nos jours, de rendre l'histoire explicative en étudiant le... nonévénementiel, et ils partagent cette ambition avec les sociologues : ils ont toujours recours à l'histoire-matériau, mais leur quête est différente (...) la sociologie est beaucoup plus qu'une excroissance de l'Histoire : elle est la manifestation d'un mode de réflexion historique : la Science Politique est l'Histoire elle-même, et l'Histoire l'intègre". (N. ROULAND, "Réflexion sur les rapports entre l'Histoire et la Science Politique", dans Le conseil municipal marseillais et sa politique de la II à la III République (Aix-en-Provence, 1974), 285, 289).

Cf. T.S. BOTTOMORE, op. cit.., 259.

Dans le même sens, cf. J. CARBONNIER, op. cit., 21.

Cf. infra, p. 119. 
cas les facultés de droit se transformeront en écoles professionnelles où le droit ne sera plus qu'une technique, un art ; ou bien le droit conquerra la place qui lui revient au sein des autres sciences humaines, auxquelles il peut apporter beaucoup par son génie classificatoire et le caractère concret des faits qu'il étudie. Dans cette optique, il n'est pas sans importance de signaler la dette déjà contractée envers les historiens du droit, comme l'écrit J. Carbonnier :

"Il n'est pas inattendu que ce soit chez les représentants des disciplines collatérales au droit, des disciplines auxiliaires, que l'idée sociologique ait éveillé dès l'abord le plus de sympathie : chez les représentants de l'histoire du droit et du droit comparé. Ils étaient déjà familiarisés avec la méthode comparative, professionnellement convaincus de la mutabilité et de la relativité du droit, en contact avec des pays étrangers où le dogmatisme était moindre autant de prédispositions à la sociologie (...). Les historiens du droit étaient, sans doute, plus de plainpied avec la sociologie, via l'ethnologie (que les comparatistes), pour peu que leur réflexion se fût portée sur un système juridique dit primitif, tel que les XII Tables ou les lois barbares" ${ }^{1}$.

De fait, chacun sait que c'est un romaniste, Henri Lévy-Bruhl, qui a pris la plus grande part à l'essor de l'ethnologie juridique, et qu'à l'heure actuelle, les historiens du droit animent encore cette discipline, en collaboration, il est vrai, avec des "littéraires" ${ }^{2}$. On notera, d'ailleurs, dans leurs écrits, une tendance très nette à donner une définition extensive de la règle de droit et à constamment insister sur les facteurs économiques et sociaux qui expliquent sa genèse (ce qui est le propre de l'attitude scientifique que nous exprimions plus haut). Quelques exemples le prouveront. [p. 113] En 1912, dans un traité qui fut longtemps fondamental, A. Esmein ne jugeait même pas utile de donner une définition de l'histoire des institutions, tant les certitudes acquises épistémologiques étaient fortes ${ }^{3}$. À l'heure actuelle, au contraire, tous les manuels y consacrent un développement et, parmi les plus récents, on note ce souci d'élargissement de la discipline. À titre d'exemple, nous citerons le manuel de J. Ellul ${ }^{4}$ :

\section{J. CARBONNIER, op. cit., 90-91.}

Sur l'histoire de l'enseignement de l'ethnologie juridique, cf. J. POIRIER, "Histoire de la pensée ethnologique", dans Ethnologie générale (op. cit.), 147 ; et du même auteur "Situation actuelle et programme de travail de l'ethnologie juridique", dans Revue Internationale des Sciences Sociales, XX 113 (1970), 515-521 ; "L'ethnologie juridique", dans Revue de l'Enseignement Supérieur, 3 (1965), 30-32. Sur celui de la sociologie juridique, cf. J. CARBONNIER, op. cit., 90-96.

3 Cf. A. ESMEIN, Cours élémentaire d'Histoire du Droit français (Paris, 1912).

4 J. ELLUL, op. cit., 5-6. Cf. aussi A. DUMAS, op. cit., 1-2, qui insiste sur le relativisme et la mutabilité de la règle de droit. Certains historiens du droit, cependant, redoutent un trop grand élargissement de la notion d'institutions : cf. P. OURLIAC, "L'objet de l'Histoire des Institutions", dans Revue Historique du Droit français et étranger (1955), 282-293, qui répond à J. 
"Le terme Institution ne doit pas être pris dans l'acception technique que ce mot a prise dans le vocabulaire juridique, ou dans la doctrine 'institutionnelle' de la philosophie du droit, mais dans une acception plus large et plus commune : tout ce qui est organisé volontairement dans une société donnée. Ceci déborde quelque peu la notion de droit (ensemble de règles assorties de sanctions établissant les rapports matériels des hommes entre eux et des hommes avec les collectivités). Mais aussi, en tant qu'Histoire, l'Histoire des institutions est différente de l'Histoire du droit à deux points de vue : d'une part, elle a pour but de décrire l'évolution des règles et des structures juridiques par rapport au contexte économique et social ; d'autre part, elle considère les phénomènes juridiques beaucoup plus dans leur essence et leur réalité profonde que dans leur manifestation technique (...) le droit ne peut être envisagé dans une histoire des Institutions, comme une réalité en soi, évoluant pour des raisons propres, et étudié d'un point de vue intrinsèque (...) le droit apparaît comme l'expression stabilisée, rationnalisée à un moment donné, de rapports sociaux et économiques, de doctrines et de tendances idéologiques, d'intervention de groupes dominants ou de personnalités décisives (...) le droit qui est l'objet de cette description est non seulement celui édicté [p. 114] par l'État, mais aussi le droit coutumier, et la pratique juridique, parfois dans son aspect le plus mouvant: par exemple celui des règles que des groupes privés suivent sans que l'on puisse parler de règles juridiques autrement qu'au point de vue sociologique, et dans la mesure où le groupe applique des sanctions, d'ailleurs spontanées, décidées par un 'pouvoir' " 1.

On voit, par là, que le champ du droit s'élargit considérablement, et peut valablement servir d'objet à la démarche sociologique et ethnologique. Un autre historien du droit, L.R. Ménager, synthétise en un discours fondamental toutes les

GODECHOT, "À propos de l'histoire des institutions : l'origine des institutions françaises de l'époque révolutionnaire", dans Revue internationale d'Histoire politique et constitutionnelle, (1951), 92-99, qui reprochait aux juristes de confondre histoire des institutions et histoire de la législation. Ce reproche d'une technicité quelque peu désincarnée n'était d'ailleurs pas récent : cf. C. APPLETON, "Notre enseignement du droit romain, ses ennemis et ses défauts", dans Mélanges Cornil (Paris, 1926), 43-79.

1 J. ELLUL, Histoire des Institutions : l'Antiquité (Paris, 1972), 5-6. M. DUVERGER, au nom de la Science Politique, affirme encore davantage le concept d'institutions, dans lesquelles il voit à la fois des "modèles structurels" non nécessairement très formalisés concrètement ("... des modèles structurels sans organisation matérielle peuvent être très cohérents ... beaucoup plus que d'autres dont les organisations matérielles apparaissent importantes... les organisations ne sont que l'écorce des institutions") et des représentations collectives : c'est la façon dont l'institution est sentie qui emporte l'adhésion du groupe social. ("Toute institution est à la fois un modèle structurel et un ensemble de représentations collectives plus ou moins valorisées".) (M. DUVERGER, Sociologie Politique (Paris, 1966), 102-104). De telles conceptions sont fort éloignées du dogmatisme juridique, mais sont bien celles d'une authentique science du droit. 
données qui précèdent, et exprime l'ambition, les méthodes et la finalité de la science du droit dans une optique historique. Malgré sa longueur, nous le citons ici in extenso, car il définit parfaitement ce que l'étude du droit ne peut plus être et ce qu'elle peut devenir :

"Une première observation s'impose, qui est d'importance. Une longue tradition s'est attachée à enfermer l'histoire des structures politiques dans les cadres étroits de la règle de droit. Conception formelle de la Rechtesgeschichte, mal dégagée de l'influence longtemps exercée par l'école historique allemande et qui repose sur la sacro-sainte et exclusive étude des textes juridiques, souveraine source de la connaissance des siècles du passé. Toute manifestation sociale s'écartant de la vérité légale y était tenue pour dirimante et devait être écartée et cachée comme une verrue. Une telle méthodologie heurte la logique la plus élémentaire et pas seulement par ses insuffisances. Son erreur fondamentale est de partir, pour reconstituer l'ordre du passé, d'un postulat aberrant qui est celui de la validité universelle et permanente de la Loi. Singulière déformation du prisme de nos mentalités modernes, qui reporte dans le passé ce qui n'a été acquis approximativement qu'après des siècles d'effort. C'est [p. 115] se faire beaucoup d'illusions sur la valeur normative de la Loi. Il eût fallu pour cela que le pouvoir fût en mesure de faire connaître, appliquer et respecter partout ses décisions. Cela mettait en jeu un appareil administratif dont les aptitudes suffisantes, les moyens adéquats et l'efficacité n'ont été que rarement les vertus dominantes.

L'une des plus profondes évidences acquises par les travaux d'histoire sociale des vingt dernières années tient sans doute dans cette opposition : la loi n'a eu qu'exceptionnellement le pouvoir de discipliner totalement les faits, au point qu'on puisse voir dans l'une le reflet exact et sincère des autres. Pour qui s'attache à l'histoire institutionnelle, le problème majeur consiste au contraire à savoir pourquoi et dans quelle mesure la réalité politique, économique et sociale n'a pas été disciplinée par la règle contraignante.

La connaissance historique ne peut qu'à ce prix gagner une certaine profondeur et, en tout cas, un peu plus d'exactitude dans l'appréciation des questions qui se posent à elle. Ce n'est pas seulement le propre du passé que les commandements ou les interdictions légales, de même que les proclamations diverses du pouvoir en soient restées à l'état de simple déclaration d'intention. Sur ce point particulier, les contradictions auxquelles mène l'observation historique objective de la vie d'hier peuvent et doivent avoir pour objet premier d'exercer la susceptibilité critique vis-à-vis des informations aujourd'hui savamment maniées par les psychotechniques pour donner de la vie de chaque jour un visage de béatitude ou de caricature.

Bref, confronter ce qui est tenu pour vrai par la norme juridique avec ce qui est effectivement pratiqué, faire naître la vérité de l'accouplement de ce que Bergson appelait la conception mécanique et la 
conception dynamique, telle est l'ambition que nous voudrions avoir pour la recherche historique.

À quoi l'appliquer, pour ce qui nous concerne ? D'abord à l'histoire, non plus, comme jadis, du 'droit public', mais des 'institutions publiques'. Cela implique-t-il un déplacement du centre de gravité de nos études ? On en a discuté, à propos de la définition même qu'il convient de donner au secteur 'institutionnel'. Le problème tombe si, en fonction de ce qui précède, on envisage les institutions publiques d'un point de vue résolument sociologique et si l'on comprend par là l'organisation des cadres dans lesquels s'exercent les activités de l'homme en tant qu'être social ou, [p. 116] comme disait Aristote, en tant qu'animal politique'.

La condition de l'homme est contingente d'une série de cercles concentriques qui, de l'extérieur, contribuent à la déterminer de plus en plus étroitement. La conjoncture extérieure et intérieure - paix ou guerre - constitue, avec les structures politiques - dictatures, monocratiques ou républiques -, les cercles les plus larges de cet ensemble. Vient ensuite l'édifice administratif qui soutient la puissance de l'État ou, au contraire, la dessert et la ruine, contribuant ou non à assurer sa domination plus ou moins étroite sur les sujets, opprime de manière variable le sort matériel des individus par sa fiscalité, les contraint par sa justice et sa police, les défend ou les asservit par son armée. Voilà qui fixe la vie et le destin des individus au sein de la Polis, de la cité, et c'est ce que nous entendons par genèse politique lorsque nous entreprenons d'en retracer les longs et difficiles cheminements.

Mais, en marge de cette détermination rigide et froide des démarches humaines, tout un ensemble de forces complexes, fluctuantes et parfois opposées agit pour accentuer ou adoucir la pression exercée sur les êtres par le schéma politique. L'Économique, d'abord, dont les contraintes peuvent faire d'un homme, libre au regard du Droit, l'esclave le plus démuni en fait. Les croyances ou les idéologies, ensuite, également aptes à soumettre les corps et à obscurcir les esprits, comme elles le sont, au contraire, à les libérer et à les élever. Les comportements sociaux, enfin, réactions spontanées et solidaires de groupes plus ou moins vastes, au moins lorsque leurs impulsions influent de manière déterminante sur les mentalités et l'action du corps social tout entier. On peut discuter à perte de vue sur ce qui est, ou plutôt sur ce qui n'est pas un 'fait social'. Composantes essentielles de l'état d'une société donnée et mobiles de son évolution, tels nous paraissent être les trois grands ordres de 'faits sociaux' que nous venons d'évoquer et dont la trame compose ce que nous entendons par genèse sociale. C'est à eux, nous semble-t-il, qu'il faut avoir recours pour compléter ou corriger, mais plus sûrement éclairer ce que la connaissance de l'histoire institutionnelle nous permet de découvrir de la condition de 
l'homme - plutôt que des hommes - dans son passé et dans son devenir" ${ }^{1}$.

\section{[p. 117]}

Il n'est nul besoin ici de se livrer à des commentaires plus développés qui ne sauraient-être que de la paraphrase, tant le texte est complet et les idées justes.

À lire tout ce qui précède, on pourrait être relativement optimiste quant à l'avenir de la science du droit et, notamment, dans ses prolongements de l'histoire, l'ethnologie et la sociologie. Mais une science qui n'est partagée que par des spécialistes est condamnée à mourir d'asphyxie : pour se développer, elle doit être enseignée. Or, par contraste avec ce mouvement d'idées, on constate que ces disciplines soit déclinent, soit stagnent, dans les programmes de l'enseignement supérieur: les attaques contre les disciplines historiques, en général, sont trop connues pour que nous y revenions; dans le domaine du droit, les réformes de l'enseignement historique sont trop souvent illusoires ${ }^{2}$, ou consistent à des suppressions pures et simples. Quant à l'ethnologie juridique, sa situation n'est guère plus brillante : peu de cours, peu de spécialistes, peu d'audience, absence de programme, écartèlement entre les facultés de droit et celles de lettres ${ }^{3}$. La sociologie juridique ne bénéficie pas non plus d'un régime de faveur : si on met à part le développement récent de la Science Politique qui bénéficie de quelques semestres d'enseignement dans les programmes de licence, la pénétration de la sociologie juridique ne s'est pas faite de façon concertée dans les [p. 118] facultés de droit, mais au coup par coup, "... à la faveur des curiosités sociologiques que pouvait éprouver tel ou tel professeur ... chacun cherchant dans une direction que lui avait suggérée sa discipline d'origine" ${ }^{4}$.

1 L.R. MÉNAGER, op. cit., 3-6. Dans le même sens, cf. A. MOMIGLIANO, "Les conséquences du renouvellement de l'Histoire des droits antiques". Communication aux Cinquièmes Rencontres des Historiens des Institutions (Lyon, 24-25 avril, 1976), à paraître dans les Actes du Colloque.

2 "On pouvait attendre que ce changement d'énoncé (l'Histoire du Droit devenant l'Histoire des Institutions) traduisit une conception nouvelle, adaptée aux bouleversements intervenus, et pas seulement dans les structures ou l'esprit des études supérieures de droit, mais dans les démarches mêmes de la science historique; au moins afin que dans les Facultés de Droit et des Sciences Économiques, Clio n'eut plus les pratiques et la réputation de Circé. Faux espoir : l'euphorie terminologique, surtout chez les juristes de ce temps, n'a jamais servi qu'à draper les vides attristants. Après un lustre de réformes sismiques, le contenu des enseignements historiques de droit est revenu, sans avoir subi le moindre froissement, se mouler dans son siège de 1920 ou 1930" (L.R. MÉNAGER, op. cit., 3).

3 Cf. Ce tableau dressé par J. POIRIER, op. cit. (Situation actuelle et programme de travail en ethnologie juridique), 520-521. On soulignera d'autant plus l'activité de M. ALLIOT et E. LE ROY au Centre d'Études Juridiques comparatives (Sorbonne) qui travaillent sur l'ethnologie juridique africaine ; de J. POIRIER à l'ancienne E.N.F.O.M. (droits africains et malgaches); et de J.N. LAMBERT (Faculté de Droit de Nice) (ethnologie juridique africaine).

4 J. CARBONNIER, op. cit., 90. Profitons-en pour souligner le rôle de pionnier qu'a joué en la matière le Pr J. CARBONNIER, privatiste, auquel nous devons le premier manuel (1972) de 
Le constat général est donc, en termes mesurés, celui d'une certaine carence qui ne correspond pas à l'évolution théorique générale. Ce n'est donc pas principalement dans une éventuelle insuffisance théorique qu'il faut en chercher les causes, mais dans un ensemble de données méthodologiques, psychologiques et historiques ${ }^{1}$.

* - Données méthodologiques : une division du travail s'est opérée entre juristes, ethnologues et sociologues qui a laissé, parfois, croire à une différence épistémologique, dont nous avons vu qu'elle était fausse. L'ethnologue, surtout lorsque son travail est le fruit d'une enquête sur le terrain prolongée, a une vision totale de la société qu'il étudie, alors que le juriste, travaillant sur documents, restreint plus ou moins consciemment son champ de vision au sujet précis qu'il étudie, d'où une tendance au dogmatisme. D'autre part, dans les faits, l'historien du droit, l'ethnologue et le sociologue privilégient certains stades culturels : l'historien du droit étudie plus volontiers les sociétés du passé de type "moderne" (étatiques), l'ethnologue les sociétés contemporaines archaïques, le sociologue les sociétés modernes du présent. Or, il ne s'agit là que de conventions de genres, n'impliquant nullement des césures épistémologiques.

* - Données psychologiques : En premier lieu, l'ethnocentrisme, conséquence inévitable du dogmatisme juridique, qui enferme le juriste dans son système national. Pour trop de juristes encore, le "vrai" droit est celui des sociétés "modernes" étatiques ou, à tout le moins, possédant un appareil institutionnel différencié, formalisé, et hiérarchisé. Pour les sociétés les plus archaïques, on parlera au mieux de pré-droit, au pire de comportement animal, biologique, spontané, rééditant ainsi les erreurs commises par Morgan à la fin du siècle dernier, sur lequel Marx s'est basé [p. 119] pour édifier toute sa théorie de l'État comme source du droit. Cette prééminence accordée à l'État n'est donc qu'une forme d'ethnocentrisme, où se rejoignent pêle-mêle "conservateurs" et marxistes.

D'autre part, l'idéalisme : le précepte de Celse, Ius est ars sequi et boni, est encore vivant dans notre mentalité juridique. Le droit est ainsi conçu comme un principe stabilisateur des rapports sociaux en vue d'un idéal moral ou religieux, alors que, bien souvent, il ne fait qu'exprimer, au contraire, des tensions socioéconomiques et la victoire toujours passagère d'un groupe social sur un autre. Étant ainsi conçu, c'est évidemment aussi de la même manière qu'il a été enseigné ... et appris. On comprendra dès lors plus facilement la difficulté qu'ont l'ethnologie et la sociologie juridiques à s'imposer chez les juristes : en privilégiant, dans l'analyse des normes juridiques, les facteurs économiques, sociaux ou politiques,

Sociologie Juridique. (Cf. aussi du même auteur, Flexible Droit (Paris, 1971). Sur la situation de l'enseignement de la sociologie juridique dans les facultés de droit, cf. J. CARBONNIER, op. cit., (Sociologie Juridique), 90-98.

1 Cf. N. ROULAND, op. cit. (Approche du phénomène juridique...), 15-22. 
elles détruisent la référence à des notions transcendantales : morale, religion, droit naturel, etc. ... C'est, en quelque sorte, la victoire du contingent sur le permanent. Et, par là, de telles démarches heurtent des convictions, troublent des habitudes mentales. D'où une réaction de rejet, ou de prudent cantonnement de ces disciplines "critiques", que l'apparence trop longtemps donnée au droit d'une technique impersonnelle, aseptisée et neutre, ne saurait tromper sur la nature des forces qui l'engendrent.

* - Données historiques. L'étude du droit a d'abord eu une finalité pratique : le droit romain, qui fut pour des siècles le système juridique le plus élaboré produit par l'humanité, est avant tout un droit procédurier. La doctrine romaine, iuris peri$t i$ et jurisconsultes, était avant tout faite de praticiens, non d'enseignants. Le droit est la technique qui sert à gagner des procès. Dans cette tradition, le droit a fort longtemps été un art ; l'idée de voir en lui une science est relativement récente. Nous avons d'ailleurs vu toutes les difficultés que beaucoup éprouvent à en tirer toutes les conséquences... De façon corollaire, l'enseignement du droit obéit à une tradition qui s'est transmise durant plus de vingt siècles, alors que l'ethnologie et la sociologie, sciences neuves n'en bénéficient (ou n'en souffrent ?) pas : à partir de cette tradition historiquement spécifique, on peut croire à une spécificité épistémologique qui, elle, n'existe pas. 
[p. 121]

\section{DEUXIÈME PARTIE}

\section{Textes inuit traduits et commentés}

\section{Chants et duels judiciaires}

Retour à la table des matières 
[p. 123]

\section{INTRODUCTION \\ DE LA DEUXIÈME PARTIE}

$\underline{\text { Retour à la table des matières }}$

Ces textes ne revêtent nullement un caractère exhaustif et ne sont que des illustrations de l'étude théorique qui précède. Ils sont divisés en sept séries.

Le texte I est d'ordre général et illustre les rapports entre la forme générale d'expression qu'est le chant dans les sociétés inuit et son utilisation dans le cadre judiciaire. Le texte II est un récit des circonstances entourant une compétition de chants. Les séries III-IV-V regroupent plusieurs textes articulés en fonction d'une distinction que nous avons établie dans notre étude théorique (cf. pp. 80-96) : certains chants, pour régler le différend, font appel à des éléments extérieurs au litige (III : chants à objet détourné) ; d'autres se réfèrent directement aux faits du litige (IV : chants à objet direct) ; certains ont un caractère mixte (V : chants à objet mixte). La série VI insiste sur certains points particuliers. La série VII donne deux exemples de chants non judiciaires.

D'autre part, ces textes ont fait l'objet de séances de travail que nous avons dirigées lors de conférences données en 1976 au Centre d'Études Arctiques (École des Hautes Études en Sciences Sociales). Leurs commentaires sont ici réduits au strict minimum.

\section{LISTE DES ABRÉVIATIONS}

K.R.P.N. Knud Rasmussen's posthumous notes on east Greenland legends and myths (M.O.G., Bd 109 nr 3, Copenhagen, 1939).

I.C.C.E. Knud RASMUSSEN, Intellectual culture of the Copper Eskimos (Rep. of the 5th Thul. Exp., IX, Copenhagen, 1932).

N.E. Knud RASMUSSEN, The Netsilik Eskimo (Rep. of the 5th Thule Exp., $\quad$ VIII - I - 2, Copenhagen, 1931). 
[p. 124]

\section{TEXTE 1 : \\ CHANTS ET DUELS JUDICIAIRES}

\section{K.R.P.N., 147-148}

$\underline{\text { Retour à la table des matières }}$

Les Inuit ont toujours été disposés à exprimer dans des chants leurs états d'âmes et leurs sentiments, leurs expériences et impressions, et toutes leurs diverses émotions.

On peut citer à ce sujet les mots d'un vieux Groënlandais de la Côte Est, Kiline :

"En l'homme, tous les chants prennent naissance dans les grandes régions vierges. Quelquefois ils nous viennent comme des pleurs, profonds comme la peine de la terre ; quelquefois comme des rires insouciants qu'on ne peut réprimer en sentant la vie et les beautés des terres du monde. Sans que nous le sachions, ils viennent avec notre souffle, ces mots et ces intonations qu'on n'emploie pas tous les jours, et ils deviennent la propriété de celui qui sait comment les chanter pour les autres".

Les sentiments qui sont ceux des fondements de la vie, tels que l'amour, la joie et l'affection du père et de la mère, trouvent leur expression dans des chants de sentiment pleins de grâce. Des sentiments plus compliqués, tels que la jalousie, l'envie, la malice, etc. engendrent les chants de satire et de dérision. En dehors des chants consacrés au divertissement et à l'amusement, on trouve une autre forme de chant, nommée uâjerutit, sorte de jeu où l'on imite les particularités des hommes ou des animaux.

Les occupations courantes donnent naissance à beaucoup de chants épiques de caractère hétérogène, décrivant la chasse dans ses diverses phases, ses joies et ses problèmes, ses luttes, ses déceptions et ses victoires, qui ont normalement pour objet de faire passer le temps pendant les longs et monotones trajets en kayak, ou chez soi dans les maisons lorsque le travail de la journée est fini. Souvent ces chants sont chantés en chœur, par exemple lorsque les chasseurs pagaient vers leurs maisons après une journée longue et difficile. 
Une forme de chant qui sort de l'ordinaire est le chant de compétition. Tous les différends sont réglés par ce moyen, quelque soit leur motif. Les protagonistes se rencontrent après s'être défiés aux endroits où les gens se rassemblaient aux différentes saisons de l'année pour leurs activités, et la dispute se déroule sous la forme d'une sorte de compétition de chants, [p. 125] dans laquelle les deux adversaires sont accompagnés par leurs groupes respectifs de supporters; au cours de cette compétition, dans le feu de l'action, les objets de controverse débouchent souvent en un combat physique, les adversaires se heurtant l'un l'autre avec leurs têtes. Celui qui se redresse en riant le plus gagne la compétition, et la partie vaincue tombe en disgrâce.

Dans le district d'Ammassalik, l'endroit où ces duels ont lieu le plus souvent est situé au bout du fjord d'Ammassalik où de nombreux umiak chargés de gens se regroupent chaque été pour faire provision de poisson pour l'hiver, et où un très grand camp de tentes est dressé à chaque fois.

Ces duels de chants qui, auparavant, constituaient les institutions judiciaires de la communauté, ont disparu depuis longtemps, car ils étaient mal vus par la Mission. Mais il reste encore des gens qui ont assisté à de telles manifestations, et qui peuvent témoigner de l'ardeur à laquelle un duel pouvait souvent parvenir, et beaucoup des vieux chants de compétition n'ont pas encore été oubliés par la nouvelle génération, qui les a reçus de ses parents.

\section{COMMENTAIRE}

Ce texte est important, parce qu'il permet de comprendre que les duels de chant ne sont qu'un aspect d'un mode d'expression polymorphe : ils peuvent servir non seulement pour régler des différends, mais pour exprimer les sentiments les plus divers.

Pourquoi recourir cependant à la forme du chant pour régler des conflits, au point que le chant revit alors, comme l'écrit K. Rasmussen, le caractère d'une institution judiciaire?

Tout d'abord parce qu'il implique une mise en forme: les arguments sont agencée, regroupés, ce qui donne au débat une cohérence (de même que nos actes de procédure obéissent à des règles très précises de composition). Ensuite, parce que dans les civilisations de culture orale le rite joue le rôle de mémoire : la répétition de formes permet de mieux retenir les arguments qui y sont soumis. Enfin, parce que la forme a une valeur sacramentelle : ce qui nécessite le recours à une forme possède déjà de ce seul fait une valeur. On a tendance à codifier, formaliser, ce qui paraît important. La répétition, le caractère incantatoire, permettent de souligner les passages les plus essentiels [p. 126] de l'argumentation. (En ce 
sens, une étude ethno-juridique de nos actes de procédure civile - dans leur aspect de formalisation - conduirait certainement à des résultats fort intéressants).

Notons aussi que L'Inuk Kiline insiste bien sur le fait que le chant exprime ce qu'il y a de plus profond, de plus mystérieux en l'individu. Là encore, le recours au chant est un détour : la forme du chant permet d'exprimer des sentiments qui autrement resteraient enfouis, étant donné le caractère "masqué" de ces sociétés inuit. Kiline dit d'ailleurs que ces chants ne sont pas la forme d'expression (mots, intonations) du langage courant. C'est la raison pour laquelle celui qui les compose devient le propriétaire de ces objets incorporels.

[p. 127]

\section{TEXTE II : UNE ÉTRANGE DANSE AU TAMBOUR}

\section{K.R.P.N., 80-88}

$\underline{\text { Retour à la table des matières }}$

Les années étaient passées rapidement, et comme elles ne semblent témoigner d'aucun évènement qui sorte de l'ordinaire, il n'y a rien à dire à leur sujet. Même la mort du père nourricier de Kunuk n'était pas mentionnée. Kunuk, ayant acquis une énorme force, ne désirait plus rester plus longtemps là où vivaient habituellement ses parents adoptifs, et, comme il souhaitait partir et vivre quelque part ailleurs, sa mère adoptive n'était que trop contente de lui manifester son accord.

Étant parti vivre en un autre endroit, Kunuk épousa une femme qui était incomparablement belle. Et étant marié, et vivant parmi d'autres gens, celle qu'il affectionnait était constamment en sa compagnie. Ceci explique qu'il était fort, et amoureux de la vie. Il s'ensuivit que lui et ses compagnons de village devinrent de bons camarades. Kunuk avait caché sa grande vigueur, si bien que personne ne savait réellement combien il était fort, bien qu'ils n'ignoraient pas complètement la force de ses bras.

Un jour, des gens arrivèrent de nombreux endroits. L'un des visiteurs cria avec entrain :

"Kunuk, Ungilagtaqut veut avoir une compétition de tambour avec toi !"

Kunuk répondit avec crainte :

- "Pourquoi donc ? Pourquoi veut-il une compétition de tambour avec moi ? "

- "Il n'a pas de véritable raison. C'est seulement la beauté de ta femme qui le fait agir !" 
- "Qu'a à voir la beauté de ma femme avec Ungilagtaqut ?"

- "Eh bien, il se trouve qu'il a trois épouses, toutes inhabituellement jolies ; il engage des compétitions de tambour contre leurs maris, et se sert d'un long couteau comme baguette de tambour, avec lequel il les poignarde mortellement : il ne rate jamais son coup ! Il te poignardera de la même façon, après quoi il prendra ta femme pour quatrième épouse".

En entendant tout ceci, Kunuk commença à se sentir anxieux, et à ne pas savoir que faire. Très déprimé, il rentra chez lui, sous sa tente. En entrant, il dit à sa vieille et sage mère nourricière :

[p. 128]

"On dit que Ungilagtaqut veut avoir une compétition de tambour avec moi, et qu'il en a à ma vie ; il va me poignarder avec son long couteau aux deux tranchants aiguisés. Après m'avoir tué, il prendra ma femme".

En écoutant cela, la vénérable vieille femme n'était ni anxieuse ni effrayée. Elle dit simplement :

- "Kunuk, il faut accepter cette compétition de tambour contre Ungilagtaqut !"

-"Comment puis-je faire ? Depuis que j'ai perdu mon père et ma mère, je n'ai jamais essayé de chanter un son de danse au tambour, et je ne sais pas non plus en composer".

- "Tu n'as pas besoin de composer de chants de danse au tambour ; apprends seulement ta berceuse, si tu la sais, cela suffira !"

Même après avoir écouté ceci, Kunuk était encore incertain, car il pensait qu'il était horrible de mourir poignardé. À la longue, sa vieille et sage mère nourricière lui dit :

"Kunuk, tu dois accepter cette compétition de tambour. Je t'ai élevé de façon à ce que tu puisses donner autant que tu reçois, car autrement tu peux t'enfuir pris par la peur".

En écoutant cela, Kunuk sentit naître en lui la colère. Étant parvenu à cet état, il commença à s'entraîner à chanter sa berceuse. Naturellement, il l'apprit tout de suite, car elle était très simple, et il restait peu de temps avant la date prévue pour la compétition de tambour.

Lorsque tout le monde eût appris ce qui arrivait à Kunuk et qu'une compétition de tambour allait se dérouler, comme d'habitude les gens ne pouvaient penser à autre chose qu'aux vêtements qu'ils allaient mettre. En effet, une compétition de tambour était un événement très rare, et personne n'aurait pensé rester chez soi. En plus il y avait dans ce cas précis quelque chose d'inhabituel en ce qui concernait les deux rivaux : la technique meurtrière qui permettait à Ungilagtaqut de se servir de son couteau tranchant, et la grande force de Kunuk, bien que ce dernier ait tout fait pour ne pas la montrer. Malgré tout, les gens se doutaient de ce dont il était capable. Pour toutes ces raisons, personne ne voulait manquer la compétition. 
Quand le désordre et le remue-ménage se furent calmés parmi les gens qui s'étaient rassemblés, Kunuk enfin se détermina à arranger les choses de telle façon que la compétition pût s'engager.

\section{[p. 129]}

Un matin très tôt, avant l'aube, beaucoup d'umiaks et de kayaks commencèrent à partir, et lorsque tous eurent quitté le rivage, Kunuk poussa son kayak à l'eau en dernier. Ce n'était pas son propre plan : il faisait seulement ce que lui avait dit sa vieille et sage mère nourricière. En se mettant dans son kayak, Kunuk avait sur lui le capuchon de son anorak, et il ne devait pas l'enlever avant que sa mère adoptive ne lui en ait donné la permission. Déjà, avant qu'elle ait poussé son kayak, elle commença à se livrer à des pratiques magiques derrière son dos. Après qu'elle ait fait ceci et qu'elle ait de nouveau chanté sa berceuse, elle lui parla en ces termes :

"Ungilagtaqut avancera sur le sol en venant directement du côté du mur. Lorsqu'il l'aura fait, tu iras tout de suite de l'autre côté. Au moment où tu apparaîtras, Ungilagtaqut commencera à chanter et à battre son tambour, et lorsqu'il aura fini, il te visera. À ce moment n'éprouve aucune peur et grandis toi autant que tu le pourras, et je suis sûre qu'il ne cherchera pas à t'atteindre à un autre endroit, il essaiera simplement de te couper au menton et de te blesser au milieu de la gorge. Lorsqu'il te lancera son arme, recroqueville toi. Si tu t'y prends ainsi, il ne pourra pas te blesser, car son arme passera au-dessus de toi. Lorsque le couteau sera planté dans le mur juste derrière ta tête, ceux qui assisteront à la compétition, s'il y en a parmi eux qui prennent ton parti, te conseilleront de le prendre. S'ils te crient de le faire, tu ne dois pas écouter leur conseil, et Ungilagtaqut reprendra son arme. Lorsqu'il aura terminé son chant pour la seconde fois, tu devras te recroqueviller, car il aura retenu ce qui se sera passé la première fois, et, et coup-ci, il visera plus bas. Alors, au moment où il jettera son arme en ta direction, tu devras sauter en l'air, car si tu fais ainsi, le couteau passera en-dessous de toi. Si alors on te dit de nouveau de prendre le couteau, tu devras t'en abstenir aussi longtemps qu'Ungilagtaqut n'aura pas essayé de le récupérer ; mais lorsqu'il tendra sa main vers le couteau, tu devras le lui retirer. Cela t'évitera de devoir aller chercher le tambour en partant du mur opposé. Lorsque tu auras le tambour, ne commence pas à en jouer, mais dis d'abord les premiers mots de la berceuse, sans placer de mélodie sur eux. Après avoir fait ainsi, abstiens-toi pendant un moment de penser à jeter le couteau sur lui. Comprends-tu bien cela ? Ne t'occupe pas de ce que l'on te pressera de faire, n'écoute [p. 130] les avis de personne. Tu dois seulement faire la chose suivante. Après un moment de silence, tu dois commencer à chanter son chant, et en faisant cela tu dois battre du tambour et danser avec un réel sérieux. Lorsque cela sera fini, et au moment où cela sera fini, tu dois lever le couteau contre lui et exactement dans la posture où tu te trouveras, lance le vers lui et essaye de l'atteindre en-dessous du menton. Si tu abats ton ennemi, tu dois tuer une chienne avec autant de facilité et, ensuite, si tu le désires, tu peux toi-même prendre une femme parmi celles dont tu as tué le mari. Maintenant, Kunuk, as-tu bien compris tous les conseils que je t'ai donnés ?" 
Kunuk répondant que oui, la vieille femme poussa avec soin son courageux fils adoptif loin du rivage.

Après avoir un peu pagayé, Kunuk regarda derrière lui et vit sa vénérable et vieille mère adoptive assise, avec le capuchon sur sa tête, à l'endroit très précis d'où il était parti. Il se rendit l'esprit apaisé à la compétition de tambour.

En arrivant au campement d'Ungilagtaqut, tous commencèrent à crier :

"Kunuk arrive pour disputer la compétition de tambour !"

Lorsque les gens eurent crié ceci, les umiaks se dirigèrent vers le rivage ; ils étaient bourrés de monde, et tous en sortirent quand les embarcations touchèrent le rivage, et ils furent bientôt debout sur la plage.

Après que tous ces gens eurent débarqué, Kunuk entra dans la maison en dernier. Lorsqu'il entra et ouvrit la porte intérieure du passage, la maison était vraiment remplie de lumière, au point que cela en était aveuglant. Dans l'entrée, il marcha du côté droit pour s'asseoir, car son puissant adversaire avait un siège sur la plate-forme près du mur de gauche. Après que Kunuk ait marché de long en large pendant un moment il s'assit contre le mur. En se préparant à s'asseoir, il chercha sa femme sans le montrer, comme vous pouvez en être sûr ! Il finit par se rendre compte qu'Ungilagtaqut l'avait déjà placée dans le coin le plus profond de sa plate-forme, où elle était assise avec un visage complètement bouleversé. Ce spectacle provoqua sa colère intérieure, mais il fit de son mieux pour qu'on ne s'en aperçoive pas et commença à entretenir l'individu qui était à côté de lui de différents sujets sans importance, bien qu'il bouille de rage en son for intérieur.

[p. 131]

Lorsqu'il y a une compétition de tambour, la coutume veut qu'on commence par faire une grande fête, mais cette fois-ci personne ne mangea rien, car les deux personnes qui devaient s'affronter n'avaient rien de drôle ou de risible à se dire ; au contraire, on s'attendait à ce que le combat se termine par la mort d'un des protagonistes.

Lorsque Kunuk eût trouvé un endroit pour s'asseoir, il enleva son anorak et l'étendit à une courte distance devant l'endroit où il devait danser. Après qu'il l'ait ôté, tout le monde brusquement cessa de parler ; constatant cela, Kunuk regarda autour de lui pour savoir quelle était la cause de ce silence soudain. Alors il vit regardez comme c'est étrange - qu'Ungilagtaqut avait déjà ses bottes et, au moment où il le regardait, il baissa les yeux sous son ventre et commença à chercher quelque chose sous la plate-forme ; très vite il en tira un grand tambour qui était fait d'une peau complètement blanche. L'ayant retiré, il le regarda un moment, le remit en place, et prit un autre tambour. Ce dernier n'était pas aussi joli, car il était souillé de sang. L'ayant sorti, il commença à mouiller la peau, et, l'ayant mouillée et prêt à avancer, il posa sa main sous la poutre du toit, et en tira un grand couteau tranchant sur ses deux côtés. En voyant cela, Kunuk se sentit un peu mal à l'aise, car il savait que tôt ou tard ce couteau s'enfoncerait dans le corps d'un d'entre eux. À la fin Ungilagtaqut se redressa, se tint un petit peu derrière la plate-forme et 
debout contre le mur. Après qu'il eût fait ceci, ses partisans commencèrent à crier :

Fais également venir ton adversaire !"

Lorsqu'ils eurent crié ceci, Kunuk aussitôt avança à l'opposé de son adversaire, contre le mur. Ceci fait, les gens commencèrent à crier et à s'amuser entre eux, et pendant ce temps, Kunuk regardait en direction de sa femme, qui commença à pleurer doucement, les trois épouses d'Ungilagtaqut l'ayant entourée dans le coin de la plate-forme. Kunuk se détourna aussitôt de ce spectacle, car lui aussi était presque sur le point de pleurer.

Alors le puissant Ungilagtaqut commença à danser, et lorsqu'il le fit, Kunuk s'efforça de tout faire pour se grandir, et comme il était déjà réellement d'une grande stature, il toucha vite les poutres du toit avec sa tête.

Lorsque Ungilagtaqut commença à chanter, ses partisans chantèrent avec lui, alors qu'aucun de ceux qui se tenaient le plus près de Kunuk ne soufflait mot. Il comprit alors qu'il pouvait compter sur eux comme supporters. [p. 132] Lorsque Ungilagtaqut eût dansé après la fin de son chant et qu'il s'arrêta, tous ses partisans qui avaient chanté avec lui se calmèrent complètement. En terminant son chant, il visa Kunuk, et tout le monde regardait le pauvre Kunuk. Mais Kunuk ne regarda pas tous ces gens qui le fixaient, car son attention était concentrée sur une chose, le lancer de couteau de son adversaire.

Ungilagtaqut lança le couteau, et au même instant Kunuk se fit tout petit, et en fait il y eut un vide au-dessus de lui. Lorsque le couteau se fût planté dans le mur, Kunuk se redressa, et à ce moment ses partisans crièrent :

"L'homme qui ne manquait jamais son coup l'a manqué ! Rends-lui la pareille, rends-lui la pareille !"

Répétant ces mots, ils entouraient Kunuk, criant dans ses oreilles ; mais il faisait semblant de ne pas entendre, conservant son regard rivé sur le couteau durant tout ce temps. Lorsque la femme de Kunuk vit cela, elle souhaita intérieurement que son mari s'en empare, mais lorsqu'elle vit qu'il ne faisait aucune tentative en ce sens, elle se demanda :

"Veut-il réellement se faire tuer ?"

Il finit par se diriger vers Ungilagtaqut et, à cet instant, les nombreuses personnes qui l'entouraient lui crièrent encore plus fort de prendre le couteau. Mais, avant qu'il pût le faire, le puissant Ungilagtaqut le saisit, retourna à sa place, et recommença à chanter et à battre du tambour, et ses partisans lui criaient :

"C'est maintenant !"

Ceux qui étaient supposés être du côté de Kunuk le regardaient en silence, et le plaignaient. Ungilagtaqut, qui n'avait jamais manqué son coup, l'avait manqué une première fois, mais ne voulait pas le manquer la seconde. 
Voyant comment les choses tournaient, la femme de Kunuk se sentait comme sur des charbons ardents, car elle était tout entière concentrée sur ce qui se passait et entièrement du côté de son mari, car elle pensait : "Maintenant, c'est comme si Kunuk était mort".

Elle se détourna de son mari pendant un moment et regarda son adversaire. Ungilagtaqut arrivait au bout de son chant, et, comme elle dési-[p. 133] rait voir une dernière fois son mari en vie, elle tourna son visage vers lui - et vit !

Comme c'est étrange ! Où était le grand Kunuk ? Là, tout contre le plancher, et il était devenu un homme minuscule, mais si ramassé et si large ! Alors après que Ungilagtaqut eût fini son chant et visé son adversaire, il jeta plusieurs coups d'œil, et il était évident qu'il ne savait pas que faire.

Après avoir repris le couteau et visé longuement, il le jeta; au moment où il faisait ce geste, Kunuk brusquement sauta en l'air et en même temps qu'il bondissait, le couteau fila entre ses jambes et on l'entendit heurter le mur. C'était la seconde fois qu'Ungilagtaqut avait manqué son coup. Voyant cela, les partisans de Kunuk crièrent d'une seule voix :

"L'homme qui ne manque jamais son coup l'a de nouveau manqué. Mets-le hors de combat, mets-le hors de combat".

Comme auparavant, Kunuk regarda le couteau un certain temps, comme pour plonger ses partisans dans l'incertitude. Sa femme commença à se demander :

"Ne le reprendra-t-il pas, cette fois ?"

Ungilagtaqut marchait déjà vers lui, et tous ceux qui avait pressé Kunuk de prendre le couteau étaient très anxieux et semblaient troublés à son sujet. Ungilagtaqut se dirigea vers Kunuk, et au moment où il avançait la main pour prendre le couteau, Kunuk l'enleva de sa main et le tint serré comme un étau. Après l'avoir pris, un petit moment se passa au bout duquel il avait aussi enlevé son tambour à Ungilagtaqut.

À ce spectacle, tout le monde se mit à crier jusqu'à ce que le bruit devienne assourdissant. Ungilagtaqut, avec un regard anxieux, retourna à sa place, avec aucune arme ou ustensile quel qu'il soit, sans couteau ni tambour. Même après qu'il eût repris sa place, le bruit continua durant longtemps, et les gens ne cessèrent de faire du bruit que lorsqu'on réclama le silence. Kunuk se mit alors à chanter et à danser après avoir d'abord joué du tambour.

Lorsque ses partisans se joignirent à son chant après qu'il eût commencé à danser et à chanter, sa voix s'éteignit. Il commença alors à danser avec application, et tout le monde pouvait pleinement se réjouir à ce spectacle.

[p. 134]

Il continua ainsi pendant longtemps, et arriva à la fin de sa berceuse. À l'approche de la fin, les nombreux spectateurs firent silence, au point qu'on aurait pu croire que la maison était vide. Lorsque Kunuk eût fini son chant, ils attendirent 
longtemps qu'il lève l'arme ; mais il continuait à jouer du tambour sans viser son adversaire ; ils lui crièrent alors tous :

"Vas-y, vise-le !"

Mais il ne le fit pas ; mais alors ils lui dirent de cesser de jouer du tambour pendant un moment. Et comme ils devenaient silencieux, il se mit à rechanter sa berceuse, et à danser avec plus d'entrain qu'avant. Maintenant la danse et le chant atteignaient un niveau réellement sérieux pour la première fois, et les autres se joignaient à lui comme auparavant. Comme il arrivait à la fin, ceux qui accompagnaient son chant se turent comme d'habitude, regardant attentivement ce qu'il allait faire. Après qu'un silence complet se fût établi, il visa son ennemi. Au même moment, Ungilagtaqut se retourna, faisant face à Kunuk. Après avoir fait ceci, il mit ses mains devant sa figure, sa main droite touchant son menton, et s'accroupit sur ses genoux, afin de se faire le plus petit possible. La bête féroce s'étant mise elle-même dans cette position, Kunuk se demanda un moment où il allait essayer de l'atteindre. À la fin, il regarda fixement un certain point, car il désirait frapper et déchirer le bord extérieur de sa main. Après l'avoir bien regardé, il lança le couteau. Je vous assure qu'il ne le manqua pas. Ungilagtaqut fut atteint au milieu de la gorge, ses mains se pressèrent contre elle, et sa gorge fut clouée au mur de la maison, exactement comme lorsque l'on tend une peau.

Au moment où cela se passait, tous dirent, en désignant Ungilagtaqut :

"Bien joué, bien joué ! Celui-là, ce mauvais individu, avait l'habitude d'être si prudent. Celui-là, ce mauvais individu, avait l'habitude d'être si prudent !"

Lorsque ce cri s'éleva, il y eut une grande agitation parmi les gens, qui maintenant étaient prêts à s'en aller. Car ils avaient très peur et souhaitaient vite partir. En peu de temps, la grande maison fut presque vide. On entendait des sanglots calmes là où était Ungilagtaqut.

Lorsque Kunuk fut prêt, il voulut s'en aller, mais d'abord il voulut prendre comme seconde femme la plus jolie des veuves d'Ungilag-[p. 135] taqut. Il passa devant son ennemi qui vivait encore, monta sur la plate-forme et approcha des trois femmes, qui s'étaient regroupées dans un coin. Après les avoir regardées un moment, il prit la plus jolie aussi facilement que si c'était un enfant et sortit avec elle ; au moment de passer devant Ungilagtaqut, il serra plus fortement son fardeau, et, en dépit de sa résistance, tourna son visage vers le sien. La tenant ainsi, il marcha lentement devant l'homme. Ungilagtaqut, rassemblant ses forces, lui dit alors :

"Tu dois ne jamais te détourner d'elle pour un long moment, car elle ne résisterait jamais si je revenais vers elle".

En entendant cela, Kunuk se pressa de sortir, et en empruntant la voie d'accès, il vit sa femme à l'autre issue, qui l'attendait. Après qu'il l'ait atteinte, ils sortirent ensemble. Ils étaient juste sortis lorsqu'ils furent accostés par surprise par quelqu'un se tenant juste à côté d'eux qui leur donna une bourrade. 
"C'était une bonne et vigoureuse bourrade !" Quoiqu'il en soit, ils ne s'y attardèrent pas la première fois, mais essayèrent de poursuivre sans regarder en arrière. Mais lorsqu'il reçut une bourrade encore plus vigoureuse, le faisant presque tomber, il se retourna. Et que vit-il ? Un petit homme, sans cou entre ses épaules et sa tête ! Lorsqu'il vit cela il chargea sa femme du fardeau qu'il transportait et lui dit de mener la femme jusqu'à l'umiak.

Les deux hommes en vinrent aux mains, et ils luttèrent dans un esprit de vengeance. Plusieurs fois Kunuk essaya de renverser son adversaire, mais ce n'était pas si facile. Le petit homme de rien du tout essayait aussi de renverser Kunuk, mais lui aussi semblait réaliser qu'il avait affaire à quelqu'un de très fort. Ces deux hommes vigoureux en vinrent bientôt à combattre réellement, et sans relâche continuèrent à lutter.

Plusieurs fois de suite, Kunuk essaya avec colère de renverser son petit adversaire, mais il échouait à chaque fois, car il avait toujours les pieds sur le sol. Juste au moment où Kunuk se sentait le moins sûr de lui, il sentit qu'il perdait pied et n'arrivait pas à garder son équilibre. Cela parce qu'il avait pris appui contre une pierre à laquelle un chien était attaché, de telle sorte qu'il glissait. Lorsque cela se passa, il lui sembla qu'il écrasait son petit adversaire. Mais comme il [p. 136] ne tombait pas et reprenait son équilibre, et était sur le point de s'accrocher très sérieusement avec l'homme, il remarqua que l'homme le poussait de façon répétée, et très prudemment il commença à relâcher son étreinte ; lorsqu'il eut fait ceci, l'homme tomba à terre. Après être tombé, le petit homme s'assit, les deux mains posées sur le sol. Encore qu'il fût perturbé, il dodelinait de la tête comme s'il dormait, et se comportait comme s'il avait perdu le sens. Voyant cela, Kunuk, sans dire un mot, le regarda.

Pendant un moment, il attendit pour voir ce qui arrivait à son adversaire. Finalement, lorsqu'il sembla être revenu à lui, il se redressa, s'assit à côté de Kunuk, et tourna sa tête vers lui. L'ayant regardé un moment, il lui dit :

"Lorsque l'habitant de la terre de l'intérieur et moi luttions et nous frappions l'un l'autre, aucun excrément ne me vint ; mais lorsque Kunuk et moi luttions et nous frappions l'un l'autre, un excrément me vint, et tout devint noir devant mes yeux car tout mon intérieur était broyé !"

Il n'y a pas de doute que ceci se produisit parce que Kunuk, lorsqu'il buta sur la pierre, pressa si fort son adversaire que quelque chose en lui se brisa et un excrément compact fut expulsé de lui. Après l'avoir regardé pendant un moment, Kunuk s'en alla sans dire un mot. Ce n'est que plus tard qu'il apprit qu'il avait lutté avec Usugssamiarssunguaq lui-même.

Maintenant que Kunuk cheminait vers la mer, il s'assura d'abord de voir si sa femme avait réellement pris sa nouvelle femme avec elle dans l'umiak. Oui, elle avait bien pensé à elle, et l'avait déjà prise dans l'umiak.

Le voyage en umiak vers la maison commença, Kunuk suivant doucement derrière l'umiak, se tenant dans son kayak comme un vainqueur. 
Arrivés chez eux, ils étaient déjà sur le rivage, quand tous crièrent :

"Kunuk a tué, Kunuk a commis un meurtre !"

À ce cri, une très vieille femme répétait continuellement :

"Que disent-ils ? Que disent-ils ?"

Mais comme ceux qui criaient ne comprenaient pas, la curieuse vieille femme ne reçut pas de réponse.

Mais quand les gens finirent par comprendre sa question, ils réjouirent son cœur en lui disant :

"Kunuk a tué !"

[p. 137]

C'étaient de très bonnes nouvelles pour la vieille femme.

Comme Kunuk approchait de l'endroit où il avait l'habitude de mettre son kayak, imaginez sa stupéfaction lorsqu'il vit la vieille femme, sa vieille mère adoptive qui n'avait pas bougé du même endroit depuis la veille, lorsqu'ils étaient partis. Il pagaya jusqu'à son endroit d'accostage habituel, et lorsqu'il y arriva sa vieille mère adoptive tira le kayak sur le rivage, et lui demanda :

"As-tu pris une femme et un chien ?"

Kunuk répondit :

"J'ai oublié, parce que j'ai eu tellement à faire que c'était terrible et désorientant".

"Cela ne fait rien, laisse", dit-elle.

Depuis ce jour, Kunuk devint célèbre, comme c'est généralement le cas.

Étant devenu si fort, il résolut de se venger prochainement de son vieil ennemi, ceci constituant son premier devoir pour le payer en retour pour ce qu'il avait fait.

\section{COMMENTAIRE}

- Le récit de cette compétition de chants combine plusieurs éléments. Plusieurs formes de compétition sont en fait mêlées. Les chants proprement dits, tout d'abord. Nous ne connaissons malheureusement pas leur contenu. La seule indication que nous ayons concerne le chant de Kunuk qui ne semble d'ailleurs pas très complexe : il s'agit d'une vieille berceuse, fort simple, que malgré son inexpérience, il est capable d'apprendre rapidement. Nous savons d'autre part que les deux protagonistes dansent et jouent du tambour. Enfin, 
ce récit semble comporter des éléments magiques et mythiques : la mère adoptive de Kunuk se livre à des opérations magiques sur son fils, elle est capable de lui prédire le déroulement du combat de façon extrêmement précise ; enfin l'épisode de la lutte avec la créature dépourvue de cou paraît avoir un caractère fabuleux.

- Le motif du duel est la possession d'une femme, l'épouse de Kunuk, ce dernier n'ayant au départ aucune intention hostile contre son adversaire. Il est important de noter qu'Ungilagtaqut ne recourt pas purement et simplement au rapt pour réaliser son désir. Il recourt à [p. 138] une procédure institutionnelle, non à une action de fait. Il faut voir là une sorte de "détour", comportement type dont nous avons souligné l'importance. Sans doute est-ce là pour Ungilagtaqut un moyen d'éviter les contestations et conflits ultérieurs qui seraient née d'un rapt: en défiant son adversaire dans des formes précises, reconnues et sanctionnées par la communauté (qui joue, conne nous allons le voir, un rôle important au cours de la compétition), il acquiert le droit légitime de prendre la femme de Kunuk en cas de victoire sur ce dernier. Comme nous l'avons souligné, ces compétitions mettent en jeu l'individu tout entier. D'ailleurs, a contrario, nous constatons que Kunuk., après avoir tué son adversaire, prend lui-même la plus bette de ses trois épouses. Ce "détour" que représente le recours à une institution formelle, fait toute la différence entre un acte purement factuel (le rapt), et une action juridique (la compétition). Il s'agit de phénomènes de nature et de conséquences différentes, même si leur finalité (la femme d'un des combattants) est la même.

- Les chants obéissent eux aussi à une procédure formelle. Kunuk est d'abord fort inquiet parce que depuis la mort de ses parents il n'a plus composé de chants, et n'a plus chanté en dansant et en s'accompagnant du tambour. Cette inquiétude est le signe de la complexité que peut revêtir cette forme de compétition, qui présente au moins deux aspects. Tout d'abord les chants ne sont pas improvisés : on sait qu'ils pouvaient se léguer (cf. texte I), Kunuk chante lui-même une vieille berceuse, il la répète durant les quelques jours qui précèdent la compétition. De plus, ils ne sont pas chantés n'importe comment : là encore Kunuk s'inquiète parce qu'il manque d'expérience, et durant la compétition, nous constatons qu'il danse et chante avec sérieux et application.

- Comme nous l'avons signalé dans notre étude, la communauté joue un rôle actif. Nous constatons d'abord que cette compétition est une fête : dès qu'on sait qu'elle va avoir lieu, tout le monde ne pense plus qu'à la question vestimentaire, ce qui prouve que c'est une occasion exceptionnelle. Cependant, contrairement à la tradition en la matière, cet évènement a un aspect tragique qui oblitère quelque peu l'aspect joyeux de la fête : d'habitude il y a un festin, mais ici ce n'est pas le cas, car tout le monde comprend que la compétition se terminera par la [p. 139] mort de l'un des combattants. Durant la compétition, l'assistance intervient activement. Elle n'est pas neutre, car chaque combattant a ses partisans. Ceux-ci lui prodiguent des conseils, ils chantent avec lui 
(ce qui prouve le caractère non improvisé des chants - que le combattant communique peut être à ses partisans avant le combat - car sinon on ne voit pas comment ils pourraient chanter des chante qu'ils ne connaissent pas par avance)! Cependant, Kunuk ne semble pas connaître à l'avance ses partisans : il ne peut les identifier qu'à partir du moment où il constate que certaine des spectateurs se taisent pendant que les partisans d'Ungilagtaqut chantent en même temps que ce dernier. Cependant, cette division de l'assistance ne dure que pendant la phase agonistique du combat : lorsqu'il est visible que Kunuk a remporté la partie, tout le monde l'approuve et condamne Ungilagtaqut en se moquant de lui. Cette observation est importante, car elle montre que la compétition, en même temps qu'elle résout le différend entre les combattants, ne se solde pas par la division du groupe. C'est ce qui lui donne un caractère d'acte de justice publique. On notera enfin qu'au sein de cette assistance très active, la femme de Kunuk demeure passive malgré l'intensité de ses émotions. Cette passivité lui est sans doute imposée par le fait qu'elle est l'objet de la compétition.

- Enfin on remarquera le formalisme de la compétition : bien que le lancer du couteau (et non les chants eux-mêmes) détermine ici l'issue du combat, il ne peut être fait qu'après une phase de chant et de danse. Ungilagtaqut ayant manqué son coup la première fois, il doit avant d'essayer de nouveau de tuer son adversaire, reprendre la procédure à son début. Kunuk se soumettra exactement à la même forme. Là encore, nous nous trouvons en présence de la forme du "détour" que nous signalions plus haut. Pour ne pas se réduire à un simple homicide et être intégré dans une procédure institutionnelle, l'acte meurtrier doit faire suite à une série d'opérations formelles, qui ont pour effet de lui donner un caractère juridique.

N.B. Il est indispensable de lire au sujet de ce chant l'article de C.Y. Charron, Le Tambour magique, un instrument autrefois utile pour la quête d'un conjoint, op. cit. L'auteur souligne les traits inhabituels de ce duel de chants et tente de les expliquer. 
[p. 140]

\section{TEXTE III : CHANTS À OBJET DETOURNÉ}

\section{TEXTE III-1}

\section{K.R.P.N., 161-164}

$\underline{\text { Retour à la table des matières }}$

Chant de compétition de Kângitsukâq contre Kûitse (lors d'une autre compétition de chant)

Je vais chanter ce chant de compétition au tambour

c'était le chant de notre père

c'était sûrement le chant de ton père

mais maintenant je vais le chanter.

Lorsque j'étais enfant et que je ne pouvais me débrouiller tout seul

il me prit en pitié, pauvre chose que j'étais,

malheureux que j'étais

maintenant que j'ai grandi

je chante contre son fils

ce menteur et ce ronchonneur - ce sage :

Une fois que je pagayais sur mon kayak

au large d'Uivfaq

je vis un phoque vraiment gros

un phoque de ma propre région

un phoque de fjord !

\section{Le chant continue en prose :}

Les phoques de fjord de mon pays sont deux fois grands comme ceux du tien. Je pagayai vers lui et lui lançai mon harpon. J'entendis alors un bruissement venant du kayak et m'aperçus que la ligne du harpon se déroulait en passant pardessous l'instrument qui me sert à lancer mes traits sur les oiseaux et qui est placé pour plus de commodité sous les lanières de fixation. Je mis alors ma pagaie dans l'eau pour stabiliser le kayak, pendant que la ligne de harpon se déroulait, mais cependant je chavirai presque lorsqu'elle se tendit. Je me fis léger et m'accrochai si fort à l'anneau qu'il me blessa, mais j'arrivai à m'y tenir.

Quelqu'un qui n'aurait pas été aussi habile

aurait difficilement pu échapper à la mort

en chavirant avec le kayak !

Mais je me tendis et tins bon.

Pendant longtemps nous ne sûmes rien des gens du Kulusuq. Mais

nous finîmes par apprendre des choses.

Il y avait beaucoup

Il y avait beaucoup de choses à ton sujet ! 
Le chant continue en prose :

[p. 141] On disait que tu avais harponné un petit phoque de fjord, deux fois moins gros que celui de mon pays, et que tu avais chaviré, avant même que la ligne ne se déroule et ne se tende. N'avais-tu pas placé ta pagaie de la bonne façon ? Ne pouvais-tu pas la coincer sous ton bras et l'appuyer sur le coin du kayak ? C'est ce qu'on fait lorsqu'on a harponné un misérable petit phoque. Bien que je ne sache pas très bien me servir de ma langue et que je ne sois pas non plus un chasseur de phoque très expérimenté, je vais t'instruire par un poème !

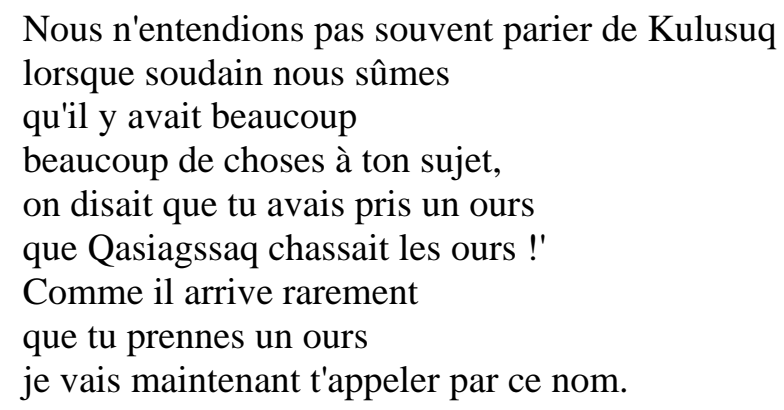

Le chant continue en prose :

Je t'appelle Qasiagssaq parce que tu te comportes comme le fait un ours lorsqu'il nage. En pagayant vers lui, tu te disais : oui, il est beaucoup plus gros que mon plus gros chien. Sur ce, ton kayak chavira, car il te frappait. Alors les autres qui étaient là se mirent à crier : l'ours a frappé Kûitse ! Lorsqu'ils t'eurent relevé, tu dis : je pensais que c'était seulement un chien, alors que c'était un ours.

Grâce aux phoques de fjord que j'ai attrapés

je peux parler contre toi, car tu ne peux pas attraper autant

de phoques de fjord que moi

j'ai pu constituer de grandes réserves d'hiver

avec les phoques de fjord que j'ai pris !

j'ai les mêmes avec les ours

que j'ai pris !

Tu n'es pas capable de prendre des ours

comme moi !

\section{COMMENTAIRE}

- On notera l'aspect héréditaire du chant, qui appartient au père des protagonistes. Kângitsukâq apparaît être seulement un fils adoptif. Cette parenté entre les deux hommes ne les empêche pas de participer à cette compétition.

- Là encore-, Kângitsukâq, comme dans le premier chant, n'évoque pas les faits précis du litige, mais cherche à ridiculiser Kûitse, en [p. 142] l'atteignant dans un domaine fort sensible : les activités de chasse. Il raconte tout d'abord un épisode, où son adversaire et lui ayant été placée dans des conditions identiques (mis à part le fait que le phoque de Kûitse était plus petit), il a fait 
preuve de son habileté, au contraire de son rival. Puis il raconte une chasse à l'ours où Kûitse paraît grotesque, car il a confondu un ours et un chien.

- Enfin Kângitsukâq dit nettement que c'est parce qu'il est meilleur chasseur que Kûitse qu'il peut le vaincre dans les compétitions de chants. 
[p. 143]

\section{TEXTE III-1 bis}

\section{K.R.P.N., 165}

$\underline{\text { Retour à la table des matières }}$

Un vieux chant au tambour (anonyme)

Regardez, regardez, cet individu

Il voudrait me défier au chant.

Lui, sur lequel j'ai fait un chant

Voudrait me défier au chant.

Lui, sur lequel j'ai composé un chant,

voudrait en composer un à mon sujet.

Chantons!

Lui, qui est arrogant avec moi, voudrait me défier au chant

Il se peut bien

que lui, à terre,

puisse plus que je ne peux.

Mais moi, pauvre malheureux,

je vais faire un poème

nous mettant tous deux en scène sur la mer

durant une grande tempête !

Il n'arriverait pas à tenir dans son kayak.

Je dois l'attacher

au phoque que je remorque

J'ai plaisir à y penser

car c'était tellement drôle

Ce n'est pas souvent qu'un chasseur habile

doit être attaché bien serré

au phoque qu'il remorque.

\section{COMMENTAIRE}

Ce chant s'apparente au second chant de Kângitsukâq contre Kûitse : l'auteur du chant cherche à ridiculiser son adversaire en le dépeignant comme un mauvais chasseur. 
[p. 144]

\section{TEXTE III-2}

\section{K.R.P.N., 159}

\section{$\underline{\text { Retour à la table des matières }}$}

Kûitse et Angitange ont échangé leurs épouses. Quand Kûitse sut que Kângitsukâq avait participé à un duel de chant avec Angitange, et que ce dernier, bien qu'il fût capable de faire des chants, n'était pas bon dans cette compétition, il en fut peiné pour lui et engagea une compétition - en son propre nom - contre Kângitsukâq. Ce dernier répondit par ce chant à Kûitse :

On dit qu'il va faire un chant satirique sur moi ;

c'est ma grand-mère paternelle

dont il s'agit.

Je ne souffrirai pas pour elle

En tant que motif de son chant satirique

Parce qu'il est navré pour lui

et veut le défendre.

La concubine

pour Angitange.

Bien, tu veux la prendre sous ta protection

Alors mets-la sur ton dos

Tu ne penses pas que tu te fatigueras

De la traiter comme une cousine

Car tu penses que si tu te fatigues d'elle

Tu la tueras.

\section{Réponse de Kûitse :}

Comme j'étais en kayak et que je pagayais

Et pendant que je pagayais sur mon kayak au nord de mon pays, au nord d'Umivik, je vis soudain un pauvre kayak dont le propriétaire ne portait pas de vêtements blancs

et qui n'était pas mince non plus

et - que vois-je - : c'était mon adversaire :

son capuchon était si noir

le long de ses joues coulaient des larmes

Oh, comme elles brillaient

En venant vers moi, pauvre malheureux, tu parlais

Faut-il prendre en pitié

Celui qui parle beaucoup?

Depuis que ma famille ne m'a plus dans sa maison 
Mais qu'elle est partie en direction du nord Je dois remercier Manîkutdlak

De m'avoir pris dans sa maison !

[p. 145]

\section{COMMENTAIRE}

On sait que l'échange de femmes est générateur d'obligations entre les participants et ne se réduit pas à la pure conjonction sexuelle. On peut penser qu'ici c'est au moins en partie à cause de ce fait que Kûitse entreprend de venger Angitange. Il y a donc là une sorte de compétition de chants par subrogation.

Là encore, nous avons affaire à des phénomènes de détour. Kûitse a brocardé Kângitsukâq en faisant allusion à sa grand-mère. Mais ce dernier, dans son chant de réponse, réoriente le débat en fonction de sa cause réelle : Kûitse l'a attaqué en raison des liens qui l'unissent à Angitange, et plus précisément à sa femme, devenue sa concubine. Kângitsukâq rétorque en disant que Kûitse n'a en l'occurrence que de mauvaises intentions : le jour où Kûitse en aura assez de cette femme, il s'en débarrassera. La réponse de Kûitse montre le désir de ce dernier de ne pas placer le débat sur ce terrain. Il se moque de Kângitsukâq en portant sur lui des appréciations globales, non pas limitées à des faits précis. Il se moque de l'apparence physique de son adversaire; de l'aspect de son kayak, et dit que ses paroles ne valent guère mieux que son aspect général. 
[p. 146]

\section{TEXTE IV : CHANTS À OBJET DIRECT. \\ TEXTE IV-1.}

\section{K.R.P.N., 168-170}

$\underline{\text { Retour à la table des matières }}$

Nutarqak chante un chant satirique contre Maratse

Je vais chanter un chant

Le chant de ton nom, Maratse.

Contre qui le chanterai-je ?

À qui l'adresserai-je?

Comme c'est le chant de ton nom, Maratse,

Je le chanterai contre toi.

Durant la saison d'été,

Hors de Nôrssît

Lorsque ta femme était partie,

et lorsque ta concubine est partie

j'entendis dire à ce sujet :

Ce Maqâq (autre nom de Maratse), que peut-il bien faire ?

Qu'a-t-il en tête ?

Prendra-t-il une femme,

Prendra-t-il une troisième femme !

Il les laissera seulement mourir de faim, même pendant les beaux jours d'été.

As-tu jamais entendu parler de cet homme

Qui a trois femmes,

mais qui les laisse mourir

même pendant les beaux jours d'été ?

Tu as parlé le premier

et tu m'as défié

Et quand je pense que tu étais celui qui m'a aidé !

Réponse de Maratse :

Laisse-moi composer un poème, laisse-moi me souvenir

que mon adversaire au duel de chant

a lui-même beaucoup d'épouses.

Je me souviens

comment mon adversaire

a pris ses femmes.

Je me souviens 
que mon adversaire

était comme un orphelin, sans père et

ses nombreuses épouses

étaient pour lui

comme des mères et des grand-mères !

Nutarqak ne perds pas de temps à faire des chants

à propos de celui qui a des épouses, celui qui en a trois,

[p. 147] auxquelles il permet juste de mourir de faim, même pendant les plus beaux jours d'été.

Je suis sûr d'avoir entendu parler de toi

J'ai entendu beaucoup de choses sur toi, que tu voulais prendre une femme, une troisième femme, mais à cause d'eux mais à cause d'Iverneq et de Pitdleqiatdiangneq tu n'as pas pu la prendre car ils avaient besoin d'elle, elle qui avait un orgelet sur l'œil elle, dont la touffe de cheveux était comme un affûteur. On dit qu'ils allèrent vers le Sud, Que Nutarqak et son groupe allèrent vers le Sud ils n'avaient pas leurs propres habits, ils n'avaient rien d'autre que des limes.

Souviens-toi maintenant, pense maintenant que c'était le milieu de l'été

et que tu te plaignais bruyamment et pleurais pitoyablement

et disais :

Vous, laissez-la ici, et apportez-la moi.

Ceci est mon chant de tambour, je me suis demandé où il devait aller, où je devais m'en servir, où il devrait s'établir, comme s'il s'agissait d'un être humain, comme si c'était un orphelin.

Où cette pauvre chose pourra-t-elle servir, où trouvera-t-elle à se reposer ?

Elle servira contre ceux contre ceux vers lesquels il faut se hâter ; contre vous, qui êtes la famille d'Ilinguakêq. Ils disent qu'il s'est moqué de moi, qu'il m'a fait passer pour fou.

Ils disent que les gens de Sermilik, et même les femmes, se réunirent 


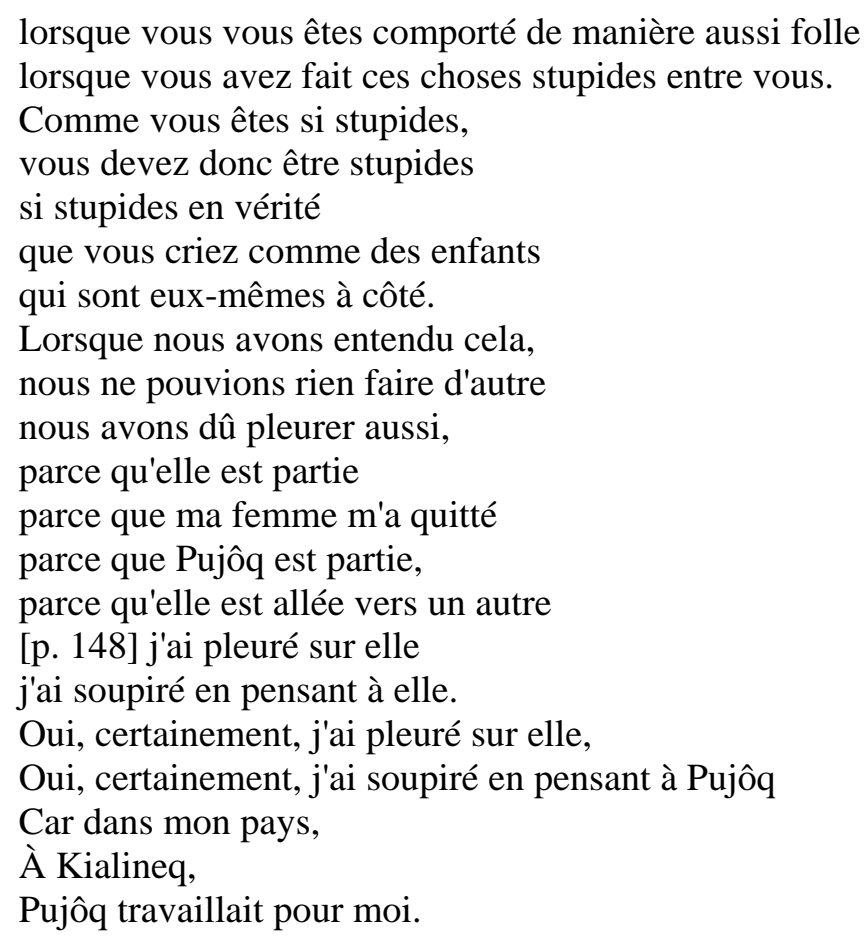

\section{COMMENTAIRE}

Ces chants sont assez exceptionnels, car ils sont pour une fois centrés sur un objet précis et ne comportent pratiquement pas de digressions.

Chacun des deux adversaires se moque de l'autre en disant qu'il n'est pas capable de prendre en charge plusieurs femmes.

Nutarqak, répondant à Maratse (nous ne connaissons pas le premier chant de Maratse) lui dit que même pendant les beaux jours d'été, il laissera ses femmes mourir de faim. Maratse lui répond en lui disant que lui-même n'est un si bon mari, puisque ses femmes le traitent comme un enfant. Puis, il dit a Nutarqak qu'il n'a d'ailleurs même pas été capable de prendre la femme qu'il voulait, encore qu'il fasse d'elle une description assez péjorative.

On voit donc que le dialogue est resté à un niveau identique : l'objet traité est la (ou les) femme(s), le thème est celui de l'incapacité de chacun des protagonistes à en trouver une ou les entretenir. 
[p. 149]

\section{TEXTE IV-2}

\section{K.R.P.N., 173-175}

$\underline{\text { Retour à la table des matières }}$

Chant de danse au tambour d'Upernâq contre Tusarpua

Upernâq, plein de pitié pour sa femme que Tusarpua a presque tué avec un couteau, chante contre ce dernier.

Parce que je ne peux pas oublier

Je vais chanter un chant au tambour.

Parce que je ne peux pas oublier

Je veux avoir une revanche.

Parce qu'il m'a presque tué en chantant,

Parce qu'il m'a presque tué,

Ce dégoûtant, cet impudent individu.

Mais je remercie

certains shamans

qui m'ont aidé jusqu'au plus profond de mon âme.

Parce que je ne peux pas oublier

Je vais maintenant chanter contre toi.

Je vais faire un chant satirique sur toi.

Car je sens une grande pitié pour (ma femme)

et je voudrais mettre à l'abri

ma pauvre femme,

ma pauvre concubine,

Tu l'as presque eue, bête sauvage.

Tu l'as presque déchirée à coups de couteau, bête sauvage.

Oui, non seulement tu l'as déchirée à coups de couteau,

mais tu l'as presque tuée, bête sauvage.

Est-ce un mauvais individu, ce Tusarpua?

Est-il un dégoûtant gros individu,

est-il un dégoûtant long individu,

gros comme un être humain,

gros comme un homme,

est-il un mauvais homme, ce Tusarpua ?

\section{Réponse de Tusarpua}

Même ceux qui sont loin disent, ceux qui vivent le plus loin à l'ouest disent que ce misérable Upernâq

pour les gens de l'Est, pour le petit nombre qu'ils sont, 


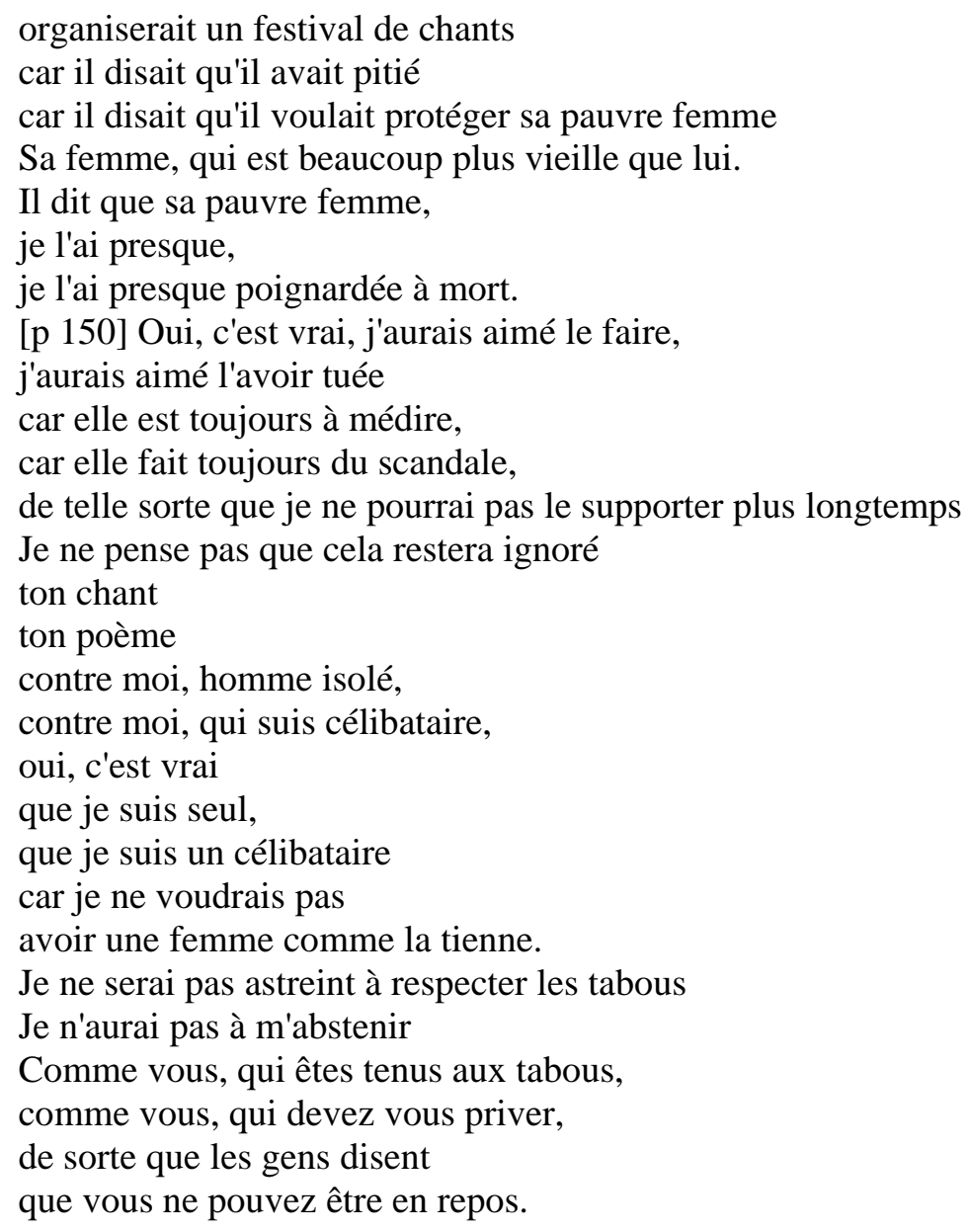

\section{COMMENTAIRE}

Ce chant est un de ceux où l'objet du litige est directement traité. Upernâq dit clairement qu'il veut se venger de Tusarpua en raison de l'agression qu'il a commise contre sa femme, et ne parle que de cela. Tusarpua accepte de répondre sur ce terrain. Il reconnaît la véracité des faits, et cherche à les expliquer d'une façon assez peu convaincante : il a essayé de tuer la femme d'Upernâq parce qu'il la trouvait insupportable. Il se livre aussi à une légère digression en s'en prenant à Upernâq, qu'il afflige de qualificatifs péjoratifs, et dont il se moque assez cruellement en faisant allusion au peu d'attrait présenté par sa ferme et aux ennuis qu'ils ont à supporter. 
[p. 151]

\section{TEXTE IV-3}

\section{K.R.P.N., 175-177}

$\underline{\text { Retour à la table des matières }}$

Chants satiriques d'Aggo contre Ilinguakêq

(Aggo était le plus réputé de tous les shamans du district d'Ammassalik)

I

Ilinguakêq a essayé de prendre comme concubine la femme d'Aggo. N'y étant pas arrivé, il a fait un chant de dérision sur elle. Aggo lui répond ainsi :

Que vais-je faire, pauvre chose ?

Je sais qu'Ilinguakêq a fait un chant de dérision sur moi.

Quelle raison penses-tu avoir d'agir ainsi ?

À quoi a bien pu se référer ton chant?

Tu n'avais pas de raison de le faire.

Peut-être penses-tu simplement que j'ai l'air d'un individu simple,

peut-être veux-tu simplement te moquer de moi ?

C'est vrai, je suis un individu modeste

C'est vrai, je suis tel qu'on peut se moquer de moi.

Je suis seul,

n'ayant ni sœurs ni frères,

personne qui m'aide.

Mais bien que vous soyez beaucoup,

je n'ai pas peur de vous

Vous ne m'intimiderez pas.

Tu es seulement un homme.

Tu n'es pas plus que moi.

II

Plus tard, la femme d'Aggo, qu'Ilinguakêq voulait pour concubine, quitta son mari pour un autre homme nommé Imâlukutsuk.

Aggo souffrait de l'avoir perdue. Ceci donna l'occasion à Ilinguakêq de chanter sur lui un chant de dérision, dans lequel il imitait les pleurs d'Aggo.

Aggo lui répondit en ces termes :

J'entendrais parler de quelqu'un

qui pleure,

alors j'éclaterai de rire

Est-ce que ce chant de tambour qui est le mien 
sera méchant, je me le demande ?

[p. 152] Sera-t-il dur, je me le demande

Pour toi, il ne sera certainement pas blessant, pour toi, il sera difficilement dur.

Mais elle, ta concubine,

La blessera-t-il, je me le demande,

lui sera-t-il dur, je me le demande?

Écoute Ilinguakêq.

Lorsque j'entendrai dire que tu es triste,

Je rirai fort,

Je me moquerai.

C'est drôle, peut-être ?

Est-ce que pleurer est drôle?

Est-ce qu'on doit se moquer de quelqu'un qui soupire ?

On raconte que vers la fin de ce chant quelqu'un se mit brusquement à crier :

"Qu'est-ce qui arrive à ce petit-là ?"

Alors celui qui avait parlé se retourna vers ce fils d'Ilinguakêq, qui était encore dans le sac à dos de sa mère, qui était en train de mourir et dont le sang coulait des coins de la bouche.

Alors lorsqu'Ilinguakêq et sa famille commencèrent à pleurer, Aggo termina son chant par un grand éclat de rire. On pense qu'en fait, Aggo, en grand shaman qu'il était, s'était arrangé avec ses esprits familiers pour que l'enfant reçoive un coup sur la tête au moment précis où il terminait son chant.

\section{COMMENTAIRE}

Dans le chant I, Aggo use du procédé familier aux Inuit de la côte Est du Groënland consistant à se déprécier eux-mêmes. Il fait une allusion intéressante au fait qu'il est seul, au contraire de son adversaire : ceci confirme que les protagonistes se présentent souvent appuyée par un groupe de supporters.

Le chant II contient une allusion identique aux individus qui ont aidé son adversaire et se sont moquée de lui. Sa façon de répondre à Ilinguakêq est assez subtile : il ne cherche pas à nier que le départ de sa ferme l'a bouleversé. Mais il s'exprime de telle façon que ce fait, au lieu de le desservir en excitant la moquerie, va au contraire montrer que les circonstances auxquelles son adversaire fait allusion ne peuvent constituer l'objet d'un chant de dérision, en raison de leur caractère tragique. Cet aspect tragique est évidemment accentué par l'événement final. 
[p. 153]

\section{TEXTE V : CHANT À OBJET MIXTE}

\section{K.R.P.N., 170-171}

\section{Retour à la table des matières}

Chant d'Atâq, appelé "Viande d'ours", contre Maratse

Le motif en est que Maratse avait fait un chant à propos de la façon dont Atâq avait mangé la chair de cadavres au cours d'une famine, et chantait ce chant devant les gens chaque fois qu'il en avait l'occasion.

Durant une compétition de chants, Atâq n'oublie pas de lui rappeler qu'il a autrefois lui-même commis un meurtre, la victime étant en fait son propre beaupère.

J'entends continuellement

le chant de mon adversaire, le chant satirique de Maratse, cela sans arrêt, à chaque fête où l'on chante.

Il a fait un chant

Il a chanté un chant satirique, à propos du temps où j'ai mangé de la chair humaine bien que j'en aie honte

alors que je ne devrais pas en avoir honte car c'était pour sauver ma vie que je le fis.

Il n'y a pas de raison que je chante une réponse parce que j'ai mangé de la chair humaine.

Mais que n'ai-je pas entendu?

Oui, bien sûr, j'ai entendu.

Maratse n'a-t-il pas commis un meurtre?

N'essaya-t-il pas de harponner son beau-père ? Mais il toucha seulement l'ouverture du kayak, il était si faible que la tête du harpon ne la transperça pas !

Écoute, Maratse !

Regarde ton beau-père, lui qui a été un père pour toi, regarde-le se traînant avec le harpon planté dans la vessie. Maratse est un individu qui n'a pas le contrôle de lui-même. Regardez, il est en train de rougir, Regardez, il est en train de changer de couleur. 
On raconte que lors de ce chant les spectateurs se mirent à hurler, et que Maratse fondit en larmes.

\section{Chant de Mitsuarnianga contre Maratse}

Je vois le compère

le compère qui est en train de ramer

[p. 154] dans le sillage de l'umiak

Il peut faire remuer sa puissante mâchoire

Comme le faisait son aïeul,

comme le faisait Taqarterajik

Taqarterajik, auquel on adressait toujours des mots, Contre lequel on dirigeait toujours des mots.

Il essaya de harponner son beau-père.

Oui, ton beau-père, qui fut un père pour toi, auquel on adressait toujours des mots, vers lequel les mots étaient toujours dirigés.

Et il voulait une troisième femme, laissez-lui en avoir une troisième, bien que certainement il soit le plus souvent incapable de trouver des peaux pour elle, et spécialement des peaux d'ours.

\section{COMMENTAIRE}

Nous avons ici l'exemple d'un chant où l'un des protagonistes use de mauvais arguments et est nettement pris en défaut.

Maratse (le perdant) commence par se signaler par un certain abus : Atâq se voit obligé de lui répondre parce que ses attaques sont continuelles. Maratse lui reproche d'avoir mangé des cadavres. Pour se disculper, Atâq va user de deux séries d'arguments.

Tout d'abord, il va montrer que l'accusation de Maratse est mal fondée. S'il a mangé de la chair humaine, c'est parce que la famine l'y obligeait, et malgré ces circonstances atténuantes, il n'en est pas particulièrement fier. Cependant, il affirme qu'il n'est pas fautif. Il est d'ailleurs vrai que si d'une façon générale les Inuit condamnent le cannibalisme, ils admettent qu'on y recoure en cas de force majeure. Atâq conclut en disant que puisqu'en définitive, il ne reconnaît pas avoir commis une faute, il n'a pas à répondre à Maratse sur ce terrain. Il se met alors à contre-attaquer, en accusant Atâq d'avoir commis, lui, une faute bien réelle en tentant d'assassiner son beau-père qui avait toujours été bon avec lui. Il en profite, au passage, pour se moquer de Maratse qui a manqué son coup à cause de sa faiblesse, ce qui est une allusion voilée à sa déficience en tant que chasseur (thème repris dans le second chant, chanté par Mitsuarnianga contre le même Maratse). On notera le rôle de l'assistance : convaincue par Atâq, elle fait bloc contre 
Maratse, qui s'effondre: il y a la un acte véritablement judiciaire à caractère public.

[p. 155] Le schéma est donc clair : Atâq reprend les faits précis de l'accusation, les réfute, et retourne la situation à son avantage en attaquant son adversaire sur un autre point.

Ce chant est donc une sorte de synthèse : il y a à la fois traitement de l'objet du litige, et déplacement du débat, phénomène que nous connaissons bien. 
[p. 156]

\section{TEXTE VI : CHANTS À CARACTÈRE PARTICULIER}

\section{TEXTE VI-1 : FAMILLE ET DUEL DE CHANTS}

\section{K.R.P.N., 166-167}

$\underline{\text { Retour à la table des matières }}$

Maratse, le shaman bien connu, chante un chant satirique contre Pêtakât qui, cette même année, dans l'été 1893, après avoir commis un meurtre fut victime d'une vendetta et fut lui-même tué.

Je perdais souffle

Je défaillais et j'étais sans force

lorsque j'atteignis le sommet de la montagne

et pus regarder vers le Sud

Il y avait de grands courants dans la mer avec beaucoup d'eau libre.

Et les terres qui s'étendaient vers le Sud

étaient voilées par la brume

et à demi-cachées par le brouillard qui montait.

Mais je pensais :

Il y a mes parents,

Il y a les côtes

Où vit mon groupe, le long desquelles ils rament doucement.

J'étais un petit peu déprimé

car je ne suis pas enthousiasmé à l'idée

de chanter contre toi,

de faire des chants contre toi

Ce qui me rend hésitant,

ce qui m'en ôte le goût,

c'est que ceux qui chantent avec nous

sont nos mères,

sont nos grand-mères,

sont notre famille,

sont nos tantes,

Ils vont chanter avec nous

bien que généralement ils ne s'aiment pas ;

lorsqu'ils s'approchent les uns des autres,

lorsqu'ils viennent les uns aux autres,

lorsqu'ils s'asseyent sur la plate-forme des uns ou des autres, 


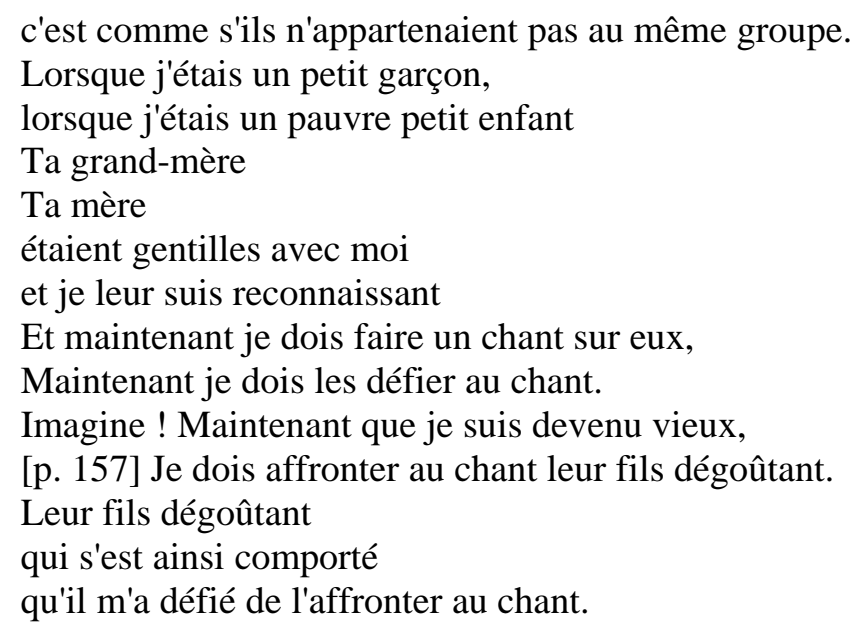

\section{COMMENTAIRE}

L'intérêt de ce chant est très net : Maratse y exprime sa réticence à engager une compétition de chants qui va diviser des individus appartenant au même groupe familial.

Dès le début du chant, Maratse fait allusion aux liens familiaux : bien que le brouillard dissimule le paysage, il sait que la terre qu'il regarde est celle où vit sa famille. Sa réticence ne provient pas de la valeur de son adversaire, puisqu'il le qualifie de "dégoutant", mais du fait que, comme nous l'avons vu, l'assistance joue un rôle actif dans les compétitions de chant et se divise en supporters de chacun des deux rivaux: or les spectateurs sont les membres de la famille des deux combattants. Maratse note tout particulièrement que le défi de Pêtakât va l'obliger à tourner en dérision la mère et la grand-mère de ce dernier qui avaient été bonnes pour lui dans son enfance. Tout ceci montre encore une fois que la compétition de chants est un acte éminemment "public" : les solidarités familiales doivent à l'occasion s'effacer devant ses nécessités (d'ailleurs Maratse nous dit que les membres de ce groupe familial, qui vont s'unir pour la compétition, n'entretiennent guère dans la vie courante de très bons rapports).

Enfin on notera que Maratse estime que pour venir à bout de Pêtakât, il va devoir s'attaquer également à sa famille : c'est encore là un aspect du caractère globalisant des compétitions de chants. 
[p. 158]

\section{TEXTE VI - 2-3 : DÉNONCIATIONS DE VOL}

\section{a) K.R.P.N., 168}

Chant satirique de Pêtakât contre Maratse, qui était connu pour ses tendances à voler.

Moi, qui suis tout tremblant,

Moi, pauvre chose, fus ébranlé

lorsque - hélas ! - je sus

par quelqu'un du Sud

contre qui je devais chanter,

c'était Maratse,

contre qui je devais chanter.

Pourquoi, pourquoi veux-tu chanter contre moi ?

Est-ce pour me remercier

que tu veux chanter contre moi ?

C'est en remerciement, parce que

tu as été jeté dehors par ceux avec qui tu habitais,

parce que ceux qui habitaient avec toi se sont débarrassés

de toi

Moi, qui t'ai trouvé une maison,

Moi, qui t'ai placé dans un endroit ?

Oui, imagine, je lui ai trouvé une maison.

Imagine, je lui ai trouvé un endroit.

Et il voudrait seulement manger,

et il voudrait seulement voler !

De qui est-il la progéniture?

Il est issu d'un corbeau !

Il n'y a que les sales corbeaux

dans ce monde

à vivre dehors,

il n'y a qu'eux dans ce monde

à se procurer de la nourriture en volant !

\section{b) -I.C.C.E., 144-145}

En visitant un village, un certain Taidluk a dérobé un couteau à neige. Personne ne sait ce qu'il en est advenu. Mais Kaufa, qui a accidentellement découvert l'objet volé, tient un meeting de dérision, au cours duquel il produit brusquement le couteau et le plante derrière lui sur le sol.

Ceci est amusant. Taidluk était celui

Par la faute duquel quelque chose manquait

C'était un couteau des gens du village 
Et il se trouva que c'était lui, Kaufa

qui, se faisant shaman, [p. 159] en le plantant sur le soi derrière lui révéla ce qui s'était passé.

Chanté par Hêq

\section{TEXTE VI-4 : CHANTS "ANORMAUX"}

\section{K.R.P.N., 173}

Chant satirique de Kunitse contre Akernilik

Lorsque Kunitse eut perdu son père, il pensa qu'Akernilik lui avait chanté un chant satirique avec une mauvaise intention.

Je suis sorti jusqu'ici avec mon pauvre chant, passant par ce chemin étroit, l'apportant avec moi, le traînant, ce rejeton de phoque tacheté, comme si je ne pouvais en prendre d'autre.

Car je ne suis pas comme lui, mon nouvel adversaire, celui qu'on appelle Akernilik, ce bon compère, ce grand chasseur.

Bien qu'il soit si intelligent, bien qu'il soit un grand chasseur, tu es beaucoup trop habile.

C'est pourquoi je t'adresse ce chant, toi qui a fait un chant satirique contre mon père, non un chant normal, mais un chant mal intentionné.

Tu viens de me le chanter.

Je peux m'occuper de moi-même, je peux chanter pour moi-même.

Oh, es-tu parti?

Bien, pars, maintenant, mais ça ne sera pour la chasse.

Après cette compétition de chants, Akernilik a commencé à mal chasser ; le dégoût de son kayak lui est venu, et il avait peur de s'aventurer au loin sur la glace. 
[p. 160]

\section{COMMENTAIRE}

L'intérêt de ce chant provient du caractère inhabituel des circonstances qui l'entourent. Il vient en réponse à un chant satirique anormal, car il allait plus loin que la simple moquerie, celui contre lequel il était dirigé étant mort. Le fils du défunt répond sur le même ton: la preuve en est qu'après ce chant Akernilik ne put plus chasser comme il le faisait auparavant. 


\section{TEXTE VII : CHANTS NON JUDICIAIRES}

\section{TEXTE VII-1 : CHANT DE PARTENAIRES DE CHANT}

N. E., 328

$\underline{\text { Retour à la table des matières }}$

Chant d'Orpingalik à son compagnon de chant.

Je me souviens de toi, mon cher compagnon de chant.

Nous étions rarement à court de mots.

Un jour un (caribou) avec d'énormes andouillers

Passe par son itinéraire,

Il passe par le grand lac.

Dans le froid qui recouvre notre kayak de glace

Lançons-nous à sa poursuite.

Avec nos deux femmes

qui regardent, lançons-nous à sa poursuite

Lequel d'entre nous parviendra derrière lui ?

Il y a longtemps à Tigdluarfik

Lorsque tu étais encore très jeune

et que les kayaks étaient là,

Ils apparurent comme une harde de loups

Près du grand lac

Tu n'allas pas assez vite

En lui suivant

Derrière moi

Je ne pouvais quand même pas t'en féliciter ! 
[p. 161]

\section{TEXTE VII-2 : MOQUERIE}

\section{K.R.P.N., 151}

Chant de désaveu (pikordlungneraineq : mention d'une incapacité).

Petit Issia, arrête de chercher une femme

Tu ne peux rien faire, arrête de chercher une femme

Il est absurde que tu veuilles un bâtard

Un habitant de l'Est qui aurait un bâtard !

Il oublie qu'il se sert d'un pot à café pour ramasser les baies

Il oublie qu'il a une peau de phoque couverte de poil sur lui

Il oublie qu'il a un énorme nez

Il oublie cela ! 
[p. 163]

\section{BIBLIOGRAPHIE}

$\underline{\text { Retour à la table des matières }}$

E. ADAMSON-HOEBEL : The Law of Primitive Man, (Cambridge, 1967). M. ALLIOT : L'acculturation juridique, dans : Ethnologie Générale, sous la dir. de J. POIRIER (Paris, 1968), 1180-1246.

P. AMSELEK: Perspectives critiques d'une réflexion épistémologique sur la théorie du droit - Essai de phénoménologie juridique (Thèse Droit Paris 1962 ; Paris, 1964).

C. APPLETON : Notre enseignement du droit romain, ses ennemis et ses défauts, dans : Mélanges Cornil, (Paris, 1926), 43-79.

R. ARON : Postface, dans : L'historien entre l'ethnologue et le futurologue, (Paris, 1972).

L. AULAGNON : Aperçu sur la force dans la règle du droit, dans : Mél. Roubier, (Paris, 1961).

A. BALIKCI : The Netsilik Eskimo, (New York, 1960).

A. BALIKCI : Development of basic socio-economic units in two Eskimo communities, dans : National Museum of Canada Bulletin, 202, (Ottawa, 1964).

L. BEAUCHET : Histoire du droit privé de la République Athénienne, I, (Amsterdam, 1969).

W. BEAUDRY : Le katajjaq comme activité ludique de la société inuit, Études Inuit/Studies, vol. 2 no. 1 (1978), 35-53.

K. BIRKET-SMITH : The Chugach Eskimo, (Copenhagen, 1953).

K. BIRKET-SMITH : Mours et coutumes des Eskimo, (Paris, 1955). M. BLOCH : La société féodale, (Paris, 1968).

F. BOAS : The Central Eskimo, (Bureau of American Ethnology, Annual Report, 1888).

T.B. BOTTOMORE : Introduction à la sociologie, (Paris, 1974).

J.L. BRIGGS : Never in Anger, Portrait of an Eskimo family, (London, 1971).

G. BURDEAU : Traité de Science Politique, I : Le Pouvoir politique, (Paris, 1966).

J. CARBONNIER : Droit Civil, I, (Paris, 1965).

J. CARBONNIER : Flexible Droit, (Paris, 1971).

J. CARBONNIER : Sociologie Juridique, (Paris, 1972). 
C.Y. CHARRON, Le tambour magique: un instrument autrefois utile pour la quête d'un conjoint, dans Études/Inuit/Studies, vol. 2 no 1 (1978), 3-20.

A. CUVILLIER : Manuel de sociologie, II, (Paris, 1968).

D. DAMAS : The diversity of Eskimo societies, dans : Man, the Hunter, (Chicago, 1968).

[p. 164

A.S. DIAMOND : L'évolution de la loi et de l'ordre, (Paris, 1954).

A. DORSINFANG-SMETS : Réflexions sur les modes de preuve dans l'action judiciaire des sociétés dites primitives, dans : Recueils de la Sté Jean Bodin : La Preuve, XVII - 3, (Bruxelles, 1963).

A. DUMAS : Manuel d'Histoire du Droit Français, (Aix-en-Provence, s.d.).

M. DUVERGER : Sociologie Politique, (Paris, 1966).

J. ELLUL : Histoire des Institutions de l'Antiquité, (Paris, 1972).

A. ESMEIN : Cours élémentaire d'Histoire du Droit Français, (Paris, 1912).

Ethnologie et Histoire, Forces productives et problèmes de transition, (Ouvrage collectif), (Paris, 1975). p. 7.

W.M. EVAN : Public and private legal systems, dans : Law and Sociology, (New York, 1972).

L. FAINBERG : On the question of the Eskimo kinship system, dans : Arctic Anthropology, IV-1, (1967).

R. FOSSIER : Histoire sociale de l'Occident Médiéval, (Paris, 1970).

P. FREUCHEN : Book of the Eskimos, (Greenwich, Conn., 1961).

J. GABUS : Vie et coutumes des Esquimaux Caribous, (Lausanne, 1944).

J. GAUDEMET : Droit Romain et coutumes malgaches, dans : Mélanges Henri Lévy-Bruhl, (Paris, 1959).

J. GAUDEMET : Institutions de l'Antiquité, (Paris, 1967).

R. GESSAIN : Ammassalik ou la civilisation obligatoire, (Paris, 1969).

J.L. GIDDINGS : 10000 Ans d'Histoire Arctique, (Paris, 1973).

M. GLUCKMANN : Politics, law and ritual in tribal society, (Oxford, 1965).

J. GODECHOT : À propos de l'histoire des institutions : l'origine des institutions françaises de l'époque révolutionnaire, dans : Revue Internationale d'Histoire politique et constitutionnelle, (1951), 92-99.

N.H.H. GRABURN : Eskimos without igloos, (Boston, 1969).

N.H.H. GRABURN : Eskimo law in light of self and group interest, dans : Law and Society Review, IV-1, (1969).

N.J. GUBSER : The Nunamiut Eskimos - Hunters of Caribou, (London, 1969).

L. GUEMPLE : Eskimo band organization and the "DP Camp" hypothesis, dans ; Arctic Anthropology, IV-2, (1972).

L. HENNIGH : Functions and limitations of Alaskan Eskimo wife trading, dans : Arctic., XXIII-I, (1970). 
E. HOLTVED : Contributions to Polar Eskimo anthropology (M.o.G., Bd. 182, no 2 ; Copenhagen, 1967).

C.C. HUGHES : Anomie, The Ammassalik, and the standardization of error, dans : Southwestern Journal of Anthropology, XIV-4, (1958).

D. JENNESS : The life of the Copper Eskimos. Rep. of the Canadian Arctic Expedition 1913-18, XII (Ottawa, 1922).

[p.165]

E.K. KANE : Arctic Explorations, (Philadelphia and London, 1856).

R. KJELLSTROM : Eskimo Marriage, (Lund, 1973).

I. KLEIVAN : Song Duels in West Greenland - Joking relationship and avoidance, dans : Folk, 13 (1971), 9-36.

H. KÖNIG : Der Rechtsbruch und sein Ausgleich bel den Eskimo, dans: Anthropos, XVIII -XIX, (1923-24) , 484-514 ; 4-5-6, 771-792 ; XX, (1925), 276-315.

H. KÖNIG : Das Recht der Polarvölker, dans : Anthropos, XXII-5-6, (Sept.-Dec. 1927).

M. LANTIS : The social culture of the Nunivak Eskimo, dans : Transactions of the American Philosophical Society, XXXV-3, (1946).

J.W. LAPIERRE: Essai sur le fondement du pouvoir politique, (Aix-enProvence, 1968).

A. LEBRUN : La Coutume, (Thèse Droit Caen, 1932).

R.B. LEE - I. DE VORE : Man, the Hunter, (Chicago, 1975).

E. LE ROY : Justice africaine et oralité juridique - Une réinterprétation de l'organisation judiciaire "traditionnelle" à la lumière d'une théorie générale du droit oral de l'Afrique Noire, dans : Bull. de L'I.F.A.N., XXXVI - B - 3 (1974), 564-569.

E. LE ROY : Cours d'Histoire des Institutions de l'Afrique Noire : I : Le Pouvoir politique en Afrique Noire, (Brazzaville, 1972-73).

E. LE ROY : Introduction aux institutions politiques africaines, (Paris I, Séminaire d'Anthropologie politique, 1975-76).

E. LE ROY : Méthodologie de l'anthropologie juridique, (Paris I, Séminaire d'anthropologie juridique, 1975-76).

E. LE ROY LADURIE : Histoire du climat depuis l'an mil, (Paris, 1967).

E. LE ROY LADURIE: Montaillou, village occitan de 1294 à 1324, (Paris, 1975).

H. LÉVY-BRUHL : L'Ethnologie Juridique, dans : Ethnologie Générale, sous la direction de J. Poirier, (Paris, 1968).

H. LÉVY-BRUHL : La preuve judiciaire chez les "primitifs", dans : Recueils de la Sté Jean Bodin : La Preuve, III, (Bruxelles, 1969).

C. LÉVI-STRAUSS : Anthropologie structurale, (Paris, 1958). 
J. MACQUERON : Histoire des obligations : le droit romain, (Publications du Centre d'histoire institutionnelle et économique de l'Antiquité classique, Série Mémoires et Travaux, I ; Aix-en-Provence, 1975).

J. MALAURIE - L. TABAH - J. SUTTER : L'isolat esquimau de Thulé, dans : Population, (oct.-déc. 1952), 675-691.

J. MALAURIE : Spécificité des sociétés esquimaudes, dans : Bulletin de la Société Royale de Géographie d'Anvers, LXXV-1-2 (1963), 9-14.

J. MALAURIE : Thèmes de recherche géomorphologique dans le Nord-Ouest du Groënland, (Paris, 1968).

J. MALAURIE : Pour une préhistoire des sociétés maritimes et de la navigation pré-esquimaude dans les zones côtières du Pacifique Nord, dans les mers de Tchoutchi et de Beaufort, dans : Inter-Nord, 12 (1972).

[p. 166]

J. MALAURIE - Y. VASARI - H. YVARINEN - G. DELIBRIAS - J. LABEYRIE : Preliminary Remarks on Holocene Paleo-climates in the regions of Thule and Inglefield Land, above all since the beginning of our own era. University of Oulu. Acta Universitatis Ouluensis : Climatic changes in Arctic Areas during the last ten thousand years, (Oulu, 1972).

J. MALAURIE : Préface à : J.L. GIDDINGS, 10000 Ans d'Histoire Arctique, (Paris, 1973).

J. MALAURIE : Les changements de climats dans le N.O. du Groënland pendant le post-glaciaire récent : nouvelles conclusions palynologiques, géomorphologiques et ethnogénétiques, dans : Les Vikings, (sous la dir. de R. Boyer), Bibliothèque arctique et antarctique, V (1974), 89-101.

J. MALAURIE : Raids et esclavage dans les sociétés autochtones du détroit de Behring, dans : Inter-Nord, 13-14 (déc. 1974).

J. MALAURIE : Les derniers rois de Thulé, (Paris, 1975).

J. MALAURIE : Les civilisations esquimaudes - Essai d'anthropogéographie, (Manuscrit déposé au Centre d'Études Arctiques).

R. MASPETIOL : La société politique et le droit, (Paris, 1957).

T. MATHIASSEN : Material culture of the Iglulik Eskimos, Rep. of the 5th Thule Expedition 1921-24, VI-I (Copenhagen, 1928).

M. MAUSS : Essai sur les variations saisonnières des sociétés Eskimos dans : L'Année sociologique, (1905) 39-132.

H., L., J. MAZEAUD : Leçons de Droit Civil, I, (Paris, 1965).

L.R. MÉNAGER : La chute de l'Empire Romain, (C.R.D.P., Marseille, 1966).

L.R. MÉNAGER : Histoire de la Propriété, (Cours de doctorat professé à Aix-enProvence, 1972).

J. MICHEA : Terre Stérile : Six mois chez les Esquimaux Caribous, (Paris, 1949).

A. MOMIGLIANO : Les Conséquences du renouvellement de l'Histoire des Droits Antiques, Communication aux Cinquièmes Rencontres des Historiens 
des Institutions, (Lyon, 24-25 avril 1976), (à paraître dans le Recueil des Actes du Colloque).

F. MOWAT : Mœurs et Coutumes des Esquimaux Caribous, (Paris, 1953).

E.W. NELSON : The Eskimo about Behring Strait, (Washington, 1899).

WENDELL H. OSWALT : Alaskan Eskimos, (San-Francisco, 1967).

P. OURLIAC : L'objet de l'Histoire des Institutions, dans : Revue Historique du Droit français et étranger, (1955).

T. PARSONS : The Law and social control, dans : Law and Sociology - Exploratory essays, (edited by W.M. Evan, New York, 1962).

E. PETITOT : Les Amérindiens du Nord-Ouest Canadien au XIX ${ }^{e}$ siècle selon E. Petitot, I : Les Esquimaux Tchiglit, (Travaux de recherches sur le delta du Mackenzie ; Ottawa, 1971).

R. PINTO - M. GRAWITZ : Méthodes des Sciences Sociales, (Paris, 1969).

[p. 167]

J. POIRIER : L'ethnologie juridique, dans : Revue de l'Enseignement Supérieur, 3 (1965).

J. POIRIER : Histoire de la pensée ethnologique, dans: Ethnologie Générale, (Paris, 1968).

J. POIRIER : Ethnologie diachronique et histoire culturelle, dans : Ethnologie Générale., (Paris, 1968).

J. POIRIER : Introduction à l'ethnologie de l'appareil juridique, dans : Ethnologie Générale, sous la direction de J. Poirier (Paris, 1968).

J. POIRIER : Situation actuelle et programme de travail de l'ethnologie juridique, dans : Revue Internationale des Sciences Sociales, XXII - (1970).

G. DE PONCINS : Kablouna, (Paris, 1947).

L. POSPISIL : The Nature of Law, dans : Transactions of the New York Academy of Sciences, Ser. II, XVIII-8 (June 1956).

L. POSPISIL : Kinship terminology and kindred among the Nunamiut Eskimo, dans : Ethnology, 11-2 (April 1963).

L. POSPISIL : Law and societal structure among the Nunamiut Eskimo, dans: Explorations in cultural anthropology, (Mac Graw Hill, 1964).

D. PRYDE : Nunaga, (Paris, 1974).

K. RASMUSSEN : Du Groënland au Pacifique, (Paris, 1929).

K. RASMUSSEN : Intellectual Culture of the Iglulik Eskimos, Rep. of the 5th Thule Expedition, VII-I (Copenhagen, 1929).

K. RASMUSSEN : Observations on the intellectual culture of the Caribou Eskimos, Rep. of the 5th Thule Exp. 1921-24, VII-2 (Copenhagen, 1930).

K. RASMUSSEN : Intellectual culture of the Iglulik Eskimos Rep. of the 5th Thule Exp. 1921-24, VII-2 (Copenhagen, 1930), 131-140. 
K. RASMUSSEN : The Netsilik Eskimos - Social life and spiritual culture, Rep. of the 5th Thule Expedition 1921-24, VIII-1/2 (Copenhagen, 1931).

K. RASMUSSEN : Posthumous notes on the life and doings of East Greenlanders in olden times (M.o.G., 109-1 ; Copenhagen, 1938).

N. ROULAND : Réflexion sur les rapports entre l'Histoire et la Science Politique, dans ; Le Conseil Municipal marseillais et sa politique de la II à la III République, (Aix-en-Provence, 1974).

N. ROULAND : Le règlement des conflits dans l'Arctique traditionnel, dans : Les Nouvelles Affiches, 1896 (déc. 1974), 2-3.

N. ROULAND : Approche du phénomène juridique dans les sociétés traditionnelles et introduction à l'étude du droit esquimau à la lumière des données comparatives fournies par les droits archaïques de l'Antiquité Occidentale, (Texte de conférences prononcées au Centre D'Études Arctiques, École des Hautes Études en Sciences Sociales, sous la direction du Pr Jean Malaurie ; Paris, 1975).

N. ROULAND : Le droit de propriété des Esquimaux et son intégration aux structures juridiques occidentales : problèmes d'acculturation juridique, dans : Actes du XLII Congrès International des Américanistes, (Paris, 1978), p. 4 n. 1.

[p.168]

N. ROULAND : Ethnologie Juridique des Inuit : approche bibliographique critique, dans : Études/Inuit/Studies, vol. II no 1 (1978), 120-131.

N. ROULAND : Cours d'Histoire du Droit français, (Aix-en-Provence, 1976).

N. ROULAND : La Convention de la baie James et les Inuit du Nouveau-Québec, (Québec, 1978).

A.J. RUBEL : Partnership and wife exchange among the Eskimo and Aleut of Northern North America, dans : Anthropological Papers of the University of Alaska, X-I (1961).

B. SALADIN D’ANGLURE : L'organisation traditionnelle des Esquimaux de Kangirsujjuaaq, (Québec, 1967).

G. SAWER : Law in Society, (Oxford, 1965).

SMIDT : p. 7.

C.M. SMIDT - I.M. SMIDT : Du chant au tambour aux cours de justice locales, dans : Objets et Mondes, XV-2 (1975), 243-246.

R.F. SPENCER : The North Alaskan Eskimo, (Washington, 1959).

G. VAN DEN STEENHOVEN : Leadership and Law among the Eskimos of the Keewatin district (N.W.T.) (Rijswijk, 1969).

G. VAN DEN STEENHOVEN : Caribou Eskimo legal concepts, dans : Legal concepts among the Netsilik Eskimos of Pelly Bay (N.W.T.), (N.C.R.C., 1959).

G. VAN DEN STEENHOVEN : Un récit historique du "bon vieux temps" chez les Esquimaux Netsilik, dans : Eskimo, 61 (mars-juin 1962). 
G. VAN DEN STEENHOVEN : The Eskimos, dans : Recueils de la Sté Jean Bodin, Gouvernée et gouvernants, XXII-I (Bruxelles, 1969).

G. STEFANI - G. LEVASSEUR : Droit Pénal Général, (Paris, 1966).

G. STEFANI - G. LEVASSEUR : Procédure pénale, (Paris, 1966).

V. STEFANSSON : My life with the Eskimo, (New York, 1922).

E.A. THOMPSON : The early Germans, (Oxford, 1965).

P. VEYNE : Comment on écrit l'Histoire, (Paris, 1971).

M. WEBER : Law in economy and society, (Cambridge, 1954).

E.M. WEYER : The Eskimos, (Archon Books, 1969).

R.M. WILLIAMS Jr. : American Society, (New York, 1951). 